\title{
When Is a Non-Markovian Quantum Process Classical?
}

\author{
Simon Milz@ , $^{1, *}$ Dario Egloff $\odot,{ }^{3,4, \dagger}$ Philip Taranto $\odot{ }^{2}$ Thomas Theurer, ${ }^{3}$ Martin B. Plenio, ${ }^{3}$ \\ Andrea Smirne $\oplus^{3,5,6}$ and Susana F. Huelga, \\ ${ }^{1}$ School of Physics and Astronomy, Monash University, Clayton, Victoria 3800, Australia \\ ${ }^{2}$ Institute for Quantum Optics and Quantum Information, \\ Austrian Academy of Sciences, Boltzmanngasse 3, 1090 Vienna, Austria \\ ${ }^{3}$ Institute of Theoretical Physics and IQST, Universität Ulm, \\ Albert-Einstein-Allee 11, D-89069 Ulm, Germany \\ ${ }^{4}$ Institute of Theoretical Physics, Technical University Dresden, D-01062 Dresden, Germany \\ ${ }^{5}$ Dipartimento di Fisica "Aldo Pontremoli," Università degli Studi di Milano, \\ via Celoria 16, I-20133 Milan, Italy \\ ${ }^{6}$ Istituto Nazionale di Fisica Nucleare, Sezione di Milano, via Celoria 16, I-20133 Milan, Italy
}

(Received 3 September 2019; revised 11 August 2020; accepted 10 September 2020; published 10 December 2020)

\begin{abstract}
More than a century after the inception of quantum theory, the question of which traits and phenomena are fundamentally quantum remains under debate. Here, we give an answer to this question for temporal processes that are probed sequentially by means of projective measurements of the same observable. Defining classical processes as those that can, in principle, be simulated by means of classical resources only, we fully characterize the set of such processes. Based on this characterization, we show that for nonMarkovian processes (i.e., processes with memory), the absence of coherence does not guarantee the classicality of observed phenomena; furthermore, we derive an experimentally and computationally accessible measure for nonclassicality in the presence of memory. We then provide a direct connection between classicality and the vanishing of quantum discord between the evolving system and its environment. Finally, we demonstrate that—in contrast to the memoryless setting—in the non-Markovian case, there exist processes that are genuinely quantum; i.e., they display nonclassical statistics independent of the measurement scheme that is employed to probe them.
\end{abstract}

DOI: 10.1103/PhysRevX.10.041049

Subject Areas: Quantum Physics, Quantum Information

\section{INTRODUCTION}

Quantum coherence is considered to be one of the fundamental traits that distinguishes quantum from classical mechanics [1-3]. Beyond its mathematical deviation from classical theory, it plays an important role in the enhancement of quantum metrology tasks [4,5], constitutes a fundamental requirement for many quantum algorithms [6,7], and has been conjectured to be necessary for the formulation of efficient transport models in biology that are consistent with spectroscopic data [8-10]. Consequently, the resource theory of coherence [11-19] has been of tremendous interest in recent years and has seen rapid development both on the theoretical and the experimental side [20].

\footnotetext{
*simon.milz@oeaw.ac.at

†dario.egloff@mailbox.tu-dresden.de

*susana.huelga@uni-ulm.de
}

Published by the American Physical Society under the terms of the Creative Commons Attribution 4.0 International license. Further distribution of this work must maintain attribution to the author(s) and the published article's title, journal citation, and DOI.
Despite such progress and the growing wealth of accompanying evidence that links coherence to nonclassical phenomena, the explicit connection between the two remains unclear and subject to active debate [21-25]. Put differently, the mere presence of coherence does not guarantee the existence of effects that cannot be explained on purely classical grounds, and an unambiguous relationship between coherence and nonclassicality has not been established yet.

In order to provide such a connection, an operationally meaningful and clear-cut definition of classicality is crucial. One such possible definition is based on experimentally attainable quantities only, namely, the joint probability distributions obtained from sequential measurements of an observable [26]. If these satisfy the Kolmogorov consistency conditions for all considered sets of measurement times - which provide the starting point for the formulation of the theory of classical stochastic processes [28,29] then they can, in principle, be explained by a fully classical model; therefore, there is nothing inherently quantum about the observed phenomenon. If they do not, then there exists no underlying classical stochastic process that could lead to the observed joint probability distributions, and the 
corresponding process is considered nonclassical. This characterization of classicality is in the spirit of the derivation of Leggett-Garg inequalities, where, instead of classicality, noninvasiveness and macroscopic realism are put to the test $[30,31]$. Indeed, any set of probability distributions that satisfies the Kolmogorov conditions does not violate the corresponding Leggett-Garg inequalities [32,33].

Following this line of reasoning, and in a sense to be further specified more precisely later, in Ref. [34] a one-toone connection was derived between the notion of classicality based on the Kolmogorov conditions and the coherence properties of the dynamics of Markovian (i.e., memoryless) quantum processes: Such processes are classical iff the corresponding dynamical propagators are unable to create coherence that can be detected at any later time. Thus, a direct relation between the mathematical notion of coherence and an operationally well-defined and broadly applicable notion of classicality has been established. In turn, this relation provides a direct interpretation of Markovian processes that violate Leggett-Garg inequalities in terms of the underlying quantum resources. However, this connection only holds in the memoryless case and does not straightforwardly apply to the nonMarkovian scenario, where, amongst other issues, such propagators cannot be used to compute multitime statistics [35].

Here, we go beyond this paradigm of memoryless processes and consider the general non-Markovian case. Such general processes can be described in terms of higherorder quantum maps, so-called quantum combs [36,37]. Recently, this framework has been tailored to the description of open quantum system dynamics $[38,39]$ and hasamongst others-found direct application in the characterization of multitime memory effects [40-43] and within the field of stochastic thermodynamics [44-46]. Here, we employ it to extend the results of Ref. [34] to the nonMarkovian case. In particular, we link spatial quantum correlations or, more precisely, the discord between an observed system and an environment, to the nonclassicality of the observed measurement statistics. Somewhat surprisingly, for the case of general processes-where memory effects play a non-negligible role - the presence of nonclassical phenomena is not solely dependent on the ability of the process to create or detect coherence, in stark contrast to the memoryless case. As we will show, the absence of detectable coherence is generally not sufficient to enforce classical behavior. Rather, classicality of multitime statistics is inherently linked to quantum discord-which was originally introduced as a means to distinguish classical spatial correlations from nonclassical ones [47-50]between the evolving system and its environment. We characterize the complete set of classical processes and derive a concrete relation between the presence and detectability of discord and the nonclassicality of observed multitime measurement statistics. This approach, in turn, allows for the derivation of experimentally accessible quantifiers of nonclassicality and the categorization of the resources required for the implementation of a nonclassical, non-Markovian process, paving the way to a clear-cut understanding of nonclassicality on operational grounds.

In a similar manner to the analysis of coherences, our results will predominantly be phrased with respect to measurements in an arbitrary, but predetermined basis, i.e., with respect to a fixed observable, raising the issue of whether classicality is merely a question of perspective; in principle, for every process, there could exist a sequential measurement scheme that yields classical statistics. While this always holds true for processes in classical physics, as well as memoryless quantum processes, we show, by means of an explicit example, that this is not necessarily the case for quantum processes with memory; in the presence of quantum memory, there exists a fundamentally new class of processes, which we will call "genuinely quantum" processes, that lead to nonclassical statistics independent of how they are probed.

Throughout this article, we investigate the question of when a physical process - with or without memory - can be considered classical and what classicality implies if we assume the underlying theory to be quantum mechanics. Concretely, for the most part, we consider the scenario of a quantum system of interest that is sequentially probed in a fixed basis, that is, interrogated at successive points in time -like, for example, in Leggett-Garg-type experimentsand we are interested in characterizing when the multitime measurement statistics resulting from such a scenario can be simulated by a classical stochastic process and thus reasonably be considered classical.

As we make no assumption about the underlying dynamics, the system of interest can be coupled to an environment that is out of the experimenter's control and can thus undergo an open evolution that displays complex classical and quantum memory effects. The classicality of the observed statistics then depends on the interplay of the dynamics of the system of interest, the pertinent memory effects, and the way in which the system is probed. We derive both the structural and the dynamical properties of general classical non-Markovian processes, providing an answer to the following question: What is a nonclassical process, and what are its key features?

Finally, by dropping the restriction to fixed instruments, we show that an observer-independent notion of nonclassicality exists, i.e., that there are processes that, no matter how they are probed, display statistics that cannot be simulated by classical stochastic processes. As such processes cannot exist in the absence of memory, the interplay of quantum memory effects and quantum dynamics leads to a fundamentally new class of processes-genuinely quantum processes - that cannot hide their nonclassicality. 


\section{SUMMARY OF MAIN RESULTS}

Before providing detailed derivations in the subsequent sections, here, we give a more concrete overview of the main results of our work. Throughout this article, we define the classicality of a process based on observed multitime statistics $\mathbb{P}_{n}\left(x_{n}, t_{n} ; \cdots ; x_{1}, t_{1}\right)$ for measurements at different times $\left\{t_{1}, \ldots, t_{n}\right\}$. The number of possible outcomes is always considered to be finite, and, unless stated otherwise, the measurements are given by measurements in the computational basis $\left\{\left|x_{k}\right\rangle\left\langle x_{k}\right|\right\}$. With respect to these statistics, a process is considered classical (on $K$ times), if the measurements made are noninvasive, i.e., if they satisfy the Kolmogorov conditions

$$
\begin{aligned}
& \mathbb{P}_{n-1}\left(x_{n}, t_{n} ; \ldots ; x_{j}, t_{j} ; \ldots ; x_{1}, t_{1}\right) \\
& =\sum_{x_{j}} \mathbb{P}_{n}\left(x_{n}, t_{n} ; \ldots ; x_{j}, t_{j} ; \ldots ; x_{1}, t_{1}\right) \quad \forall n \leq K, \quad \forall j .
\end{aligned}
$$

On the other hand, it is Markovian, i.e., memoryless, if the respective conditional probabilities satisfy

$$
\mathbb{P}\left(x_{n} \mid x_{n-1}, \ldots, x_{1}\right)=\mathbb{P}\left(x_{n} \mid x_{n-1}\right) \quad \forall n \leq K .
$$

In quantum mechanics, such a process can be modeled by means of completely positive trace-preserving (CPTP) maps $\left\{\Lambda_{t_{j}, t_{j-1}}\right\}$, which act on the probed system and describe the dynamics between measurements, as well as an initial system state $\rho_{t_{0}}$.

Going beyond the results of Ref. [34], we show that (see Theorem 1) a Markovian process is classical iff it can be modeled by a state $\rho_{t_{0}}$ that is diagonal in the measurement basis $\left\{\left|x_{k}\right\rangle\left\langle x_{k}\right|\right\}$ and non-coherence-generating-anddetecting (NCGD) maps $\Lambda_{t_{k}, t_{k-1}}$, i.e., maps that satisfy

$$
\begin{aligned}
& \Delta \circ \Lambda_{t_{j+1}, t_{j}} \circ \Delta \circ \Lambda_{t_{j}, t_{j-1}} \circ \Delta \\
& \quad=\Delta \circ \Lambda_{t_{j+1}, t_{j}} \circ \Lambda_{t_{j}, t_{j-1}} \circ \Delta \quad \forall j,
\end{aligned}
$$

where $\Delta$ is the completely dephasing map in the measurement basis and $\circ$ denotes composition. Intuitively, maps that satisfy the above equation can create coherences but not in a way that can be detected at a later time by means of the employed measurement basis. Thus, Theorem 1 provides a direct connection between coherence and an experimentally testable notion of classicality in the Markovian case.

Going beyond the Markovian case, we show that this direct connection between coherence and classicality breaks down when memory is present. We provide an explicit example (Example 1) of a dynamics $U_{t_{j}, t_{i}}|\ell, p\rangle=$ $e^{i \phi_{\ell} p\left(t_{j}-t_{i}\right)}|\ell, p\rangle$ acting on a qubit system (represented by $\ell$ ) coupled to a continuous degree of freedom (represented by p) that - for the right choice of initial environment state- never displays coherences in the system state but exhibits nonclassical statistics nonetheless.

When memory plays a non-negligible role, individual CPTP maps that act on the system alone are insufficient for the computation of multitime probabilities. Rather, probabilities are computed by means of higher-order quantum maps, called quantum combs [37,51]. These maps contain all information about the underlying process at hand, and multitime joint probabilities can then be expressed as

$$
\mathbb{P}_{K}\left(x_{K}, t_{K} ; \ldots ; x_{1}, t_{1}\right)=\mathcal{C}_{K}\left[\mathcal{P}_{x_{K}}, \ldots, \mathcal{P}_{x_{1}}\right],
$$

where $\mathcal{C}_{K}$ is the quantum comb of the process and $\left\{\mathcal{P}_{x_{j}}\right\}$ are the CP maps corresponding to measurements with outcome $x_{j}$, i.e., $\mathcal{P}_{x_{j}}[\rho]=\left\langle x_{j}|\rho| x_{j}\right\rangle\left|x_{j}\right\rangle\left\langle x_{j}\right|$.

We derive a full characterization of combs that lead to classical statistics in Theorem 2 and make this characterization more concrete in Theorem $2^{\prime}$, employing the ChoiJamiołkowski isomorphism (CJI) that allows one to map higher-order quantum maps $\mathcal{C}_{n}$ onto multipartite quantum states $C_{n}$.

Using this full characterization, a measure $M(C)$ for the nonclassicality of a process $C$ can be derived. We phrase this problem in terms of the operational task of deciding whether or not a given comb $C$ is classical, and we show that the corresponding maximum probability to guess correctly is given by [see Eq. (54)]

$$
\mathbb{P}(C)=\frac{1}{2}(1+M(C)),
$$

where $M(C)$ can be computed efficiently via a linear program [see Eq. (56)] and is accessible experimentally - and could be evaluated based on already-existing experimental data (e.g., in Ref. [52]). We show that, e.g., in the two-time case,

$$
M(C) \leq \sum_{x_{2}}\left|\mathbb{P}\left(x_{2}\right)-\sum_{x_{1}} \mathbb{P}\left(x_{2}, x_{1}\right)\right|
$$

holds, where the right-hand side of the above equation is a natural quantifier of classicality, which is used both theoretically and experimentally (for example, in Leggett-Garg-type scenarios) to quantify the nonclassicality of sequential measurement statistics.

In the same vein as in the Markovian case, the dynamical properties (in contrast to the aforementioned structural ones) of classical processes can be obtained. In the nonMarkovian case, a process is fully defined by an initial system-environment state $\eta_{t_{0}}^{\text {se }}$ and intermediate systemenvironment CPTP maps $\Gamma_{t_{j}, t_{j-1}}$. We show that in the non-Markovian case, rather than the coherences of the system, it is the (basis-dependent) system-environment discord [47-50] that determines the classicality of the observed statistics. In particular, we demonstrate (see 
Theorems 3 and 4) that a process is classical iff it can be modeled by an initial state $\eta_{t_{0}}^{s e}$ with vanishing (basisdependent) discord, i.e., $\eta_{t_{0}}^{s e}=\sum_{m} p_{m}\left|x_{m}\right\rangle\left\langle x_{m}\right| \otimes \xi_{m}$, and a set of system-environment maps that are nondiscord-generating-and-detecting (NDGD), i.e.,

$$
\begin{aligned}
& \Delta \circ \Gamma_{t_{j+1}, t_{j}} \circ \Delta \circ \Gamma_{t_{j}, t_{j-1}} \circ \Delta \\
& \quad=\Delta \circ \Gamma_{t_{j+1}, t_{j}} \circ \Gamma_{t_{j}, t_{j-1}} \circ \Delta,
\end{aligned}
$$

where the completely dephasing map $\Delta$ acts on the system alone. Analogously to the Markovian case, the above equation implies that the maps $\left\{\Gamma_{t_{j}, t_{j-1}}\right\}$ can create discord, but said discord cannot be detected by means of later measurements on the system in the chosen measurement basis. In turn, this result provides a direct connection between quantum discord and the classicality of a quantum process. Additionally, it also gives an a posteriori explanation for why the absence of coherence in Example 1 does not lead to classical statistics (for an explicit discussion of the discord that leads to nonclassical statistics in Example 1, see its continuation in Example $1^{\prime}$ ).

While, in principle, these aforementioned results do not rely on the fact that we assume measurements in one fixed basis-but could similarly be obtained for different (but fixed) instruments at every time-they still depend on the fact that one specific measurement scheme is chosen beforehand. Classicality (or the absence thereof) of the observed statistics could thus depend on the respective choice of measurement schemes. This statement holds true in the Markovian case, where there is always a choice of measurement bases that renders the observed statistics classical. However, as we show by explicit example (see Sec. VII), there are processes with memory-dubbed genuinely quantum-that display nonclassical statistics independent of the employed measurement scheme.

The paper is structured as follows: In Sec. III, we introduce the basic concepts that will be employed throughout this article to examine classicality. In Sec. IV, we reiterate and slightly generalize the results of Ref. [34], linking nonclassicality and coherence for the Markovian case, and discuss their breakdown when memory effects are present. This discussion motivates our consideration of the non-Markovian case in Sec. V, where we fully characterize the set of general classical processes by means of the quantum comb framework. This characterization then enables us to formulate a quantifier of nonclassicality that is both experimentally accessible and can be computed efficiently. Based on these results, in Sec. VI, we subsequently establish the direct connection between (basis-dependent) quantum discord and the classicality of temporal processes. Finally, in Sec. VII, we go beyond the paradigm of measurements in a fixed basis and provide an example for processes that appear quantum independent of the scheme that is used to probe them. The paper concludes in Sec. VIII with a summary and an outlook on further research directions and open problems.

\section{GENERAL FRAMEWORK}

The overarching aim of this paper is to characterize when a general quantum mechanical process can be considered classical in an operationally consistent manner and to identify the structural properties consequently implied on the underlying evolution. Importantly, our investigation will be operational in the sense that it is based solely on experimentally accessible quantities; as such, it applies to situations where the underlying theory is classical mechanics, quantum mechanics, or some more general theory [53].

Ultimately, any physical theory provides predictions about possible observations - only these can be tested by experiments. In other words, any theory must (in principle) provide the correct probabilities for measurement outcomes (or sequences thereof) to occur when a system of interest is experimentally probed. The difference between predictions made regarding such observable quantities by classical physics and quantum (or postquantum) theory can then be used to unambiguously demarcate between the theories on the investigated spatial and temporal scales.

Following Ref. [34], we thus define our notion of classicality by means of joint probability distributions pertaining to sequences of measurement outcomes, as these are precisely what is obtained when a temporal process is probed.

\section{A. Kolmogorov conditions and classicality}

In classical physics, a stochastic process on a set of $K$ times is fully described by a joint probability distribution

$$
\mathbb{P}_{K}\left(x_{K}, t_{K} ; \ldots ; x_{1}, t_{1}\right),
$$

which yields the probability to measure the realizations $\left\{x_{K}, \ldots, x_{1}\right\}$ of the random variables $\left\{X_{K}, \ldots, X_{1}\right\}$ at times $\left\{t_{K}, \ldots, t_{1}\right\}$. For example, $\mathbb{P}_{2}\left(x_{2}, t_{2} ; x_{1}, t_{1}\right)$ could describe the probability to obtain both outcomes $\left\{x_{2}, x_{1}\right\}$ when measuring the position of a particle undergoing Brownian motion at times $t_{1}$ and $t_{2}>t_{1}$. In what follows, we will often omit the explicit time label, with the understanding that $x_{j}$ denotes an outcome of a measurement at time $t_{j}$.

Crucially, in classical physics, joint probability distributions describing a stochastic process for different sets of times satisfy the so-called Kolmogorov consistency conditions $[28,29,54,55]$ : Given a joint probability distribution $\mathbb{P}_{K}$ for a set of times, the probability distributions for all subsets of times can be obtained by marginalization, that is,

$$
\begin{aligned}
& \mathbb{P}_{n-1}\left(x_{n}, t_{n} ; \ldots ; x_{j}, t_{j} ; \ldots ; x_{1}, t_{1}\right) \\
& \quad=\sum_{x_{j}} \mathbb{P}_{n}\left(x_{n}, t_{n} ; \ldots ; x_{j}, t_{j} ; \ldots ; x_{1}, t_{1}\right) \quad \forall n \leq K, \quad \forall j .
\end{aligned}
$$


Just like the Leggett-Garg inequalities [30-32] for temporal correlations, the satisfaction of these requirements is based on the assumptions of realism per se, i.e., the assumption that $x_{j}$ has a definite value at any time $t_{j}$, and the possibility to implement noninvasive measurements [56].

Importantly, an experimenter obtaining a family of joint probability distributions that satisfies the Kolmogorov conditions when probing a temporal process at different sets of times would not be able to distinguish said process from a classical one, as every such finite family can be obtained from a-potentially exotic — underlying classical stochastic process. More generally, the Kolmogorov extension theorem states that if all joint probability distributions for finite subsets of a time interval $[0, t]$ satisfy the consistency conditions of Eq. (9) amongst each other, then there exists an underlying classical stochastic process on said time interval that leads to the observed probability distributions [28,29,54,55]. In other words, if the Kolmogorov consistency conditions of Eq. (9) are satisfied (for all considered choices of $t_{j}$ ), then there is nothing inherently quantum mechanical about the observed process. We therefore give the following definition:

Definition 1. ( $K$-classical process [34]) Let $\mathcal{X}$ be a finite set. A process defined on a set of times $\mathcal{T}$, with $|\mathcal{T}|=K$, that is described by the joint probabilities $\mathbb{P}_{n}\left(x_{n}, t_{n} ; \ldots ; x_{1}, t_{1}\right)$, with $t_{n} \geq \ldots \geq t_{1}, t_{i} \in \mathcal{T}, n \leq K$, and $x_{i} \in \mathcal{X}$, is said to be " $K$ classical" if the Kolmogorov consistency conditions of Eq. (9) are satisfied up to $n=K$.

Throughout this article, we call a family of joint probabilities on a set of $K$ times a " $K$ process" and denote it by $\left\{\mathbb{P}_{n}\left(x_{n}, \ldots, x_{1}\right)\right\}_{n \leq K}$. Here, the label $n \leq K$ is a shorthand notation for all the subsets of $\mathcal{T}$ with $n$ ordered times $t_{n} \geq \ldots \geq t_{1}$, where $t_{i} \in \mathcal{T}$, for any $n \leq K$; moreover, from here on, we will not explicitly indicate the time arguments in the probability distributions, implying that the outcome $x_{j}$ refers to time $t_{j}$.

While the above definition of classicality seems intuitive, some comments are in order. First, we choose to define classicality for a finite set of $K$ times. While this choice is motivated on practical grounds, the general definition of a classical stochastic process involves the joint probability distributions associated with any number of ordered time instants $t_{K} \geq \ldots \geq t_{1}$, with $K \in \mathbb{N}$, and any choice of such instants. In particular, as already mentioned, the Kolmogorov extension theorem infers the existence of a stochastic process from the validity of the consistency conditions on all such joint distributions. Here, instead, we fix a finite value of $K$ and the sequence of time instants beforehand so that, given the $K$-time joint probability distribution of a $K$-classical process, the involved hierarchy of probability distributions can be constructed by iteratively applying the consistency conditions at any intermediate time.

Second, the above definition of classicality is a priori device independent, as it only relies on the inferred statistics without any assumptions on the underlying theory and/or measurement devices; as a consequence, the classicality of a process according to the above definition depends upon the manner in which the system of interest is probed. Although often overlooked, this is also the case in classical physics: Given some underlying classical stochastic process, not every set of measurements that an experimenter might be able to perform will lead to a family of probability distributions that satisfy the above definition of $K$ classicality. In fact, if performing such measurements might potentially disturb the system (i.e., the measurement is invasive), the Kolmogorov condition fails, in general, even if the underlying evolution is classical [56].

For example, suppose that instead of merely measuring the position of a particle at different times when probing a Brownian motion process, an experimenter chooses to displace the particle at each time depending on where it was found. In this case, Eq. (9) would generally fail to hold for the joint probability distributions observed. Consequently, the Kolmogorov consistency conditions in Eq. (9) are in fact a statement of the noninvasiveness of the performed measurements: If they hold true, then not performing a measurement at any given time cannot be distinguished (for the given experimental situation) from averaging over their probabilities (i.e., forgetting the outcomes of the measurements performed).

In classical physics, one assumes that, in principle, one could measure the system without disturbing it and that, therefore, there exists a family of joint probability distributions that can consistently explain all possible outcome probabilities. Such a noninvasive and complete measurement is often referred to as an "ideal measurement" in the literature [57].

On the other hand, in quantum mechanics, any measurement disturbs some system state, and therefore, ideal measurements do not exist, in general, in the strong sense discussed above. As a consequence, quantum mechanical processes generically do not satisfy Kolmogorov conditions [56,58], a fact that fundamentally distinguishes them from the classical realm.

More generally, the violations of Bell, Kochen-Specker, or Leggett-Garg inequalities, which can be observed in quantum mechanics, are different manifestations of the impossibility to obtain the measured data by noninvasive measurements. Particularly, in the case of Leggett-Garg inequalities [30,59], it is precisely the breakdown of Kolmogorov conditions that is being probed $[34,56]$, and our above definition of classicality is hence in line with the wider program of determining fundamentally quantum traits of nature.

\section{B. Measurement setup}

As mentioned above, the structural properties of families of joint probability distributions depend on the way in which a system of interest is probed. Consequently, before being able to analyze the set of quantum processes, it is 
crucial to fix the measurements that are used to probe a process at hand. Although there are no ideal measurements in quantum mechanics, projective measurements share some basic features with the classical ideal measurements discussed above and are thus a natural choice. In particular, they guarantee repeatability, i.e., that two sequential measurements (without any evolution in between) would give the same value with unit probability, as well as a weaker form of ideality, namely, that if an outcome occurs with certainty, then the state of the system before the measurement is not disturbed by the latter [60]. Therefore, it suggests starting our analysis on the classical reproducibility of quantum processes by focusing on projective measurements; moreover, also following Ref. [34], we further restrict ourselves to the case of orthogonal rank-1 projectors, like, e.g., projective measurements with respect to the eigenbasis of any nondegenerate self-adjoint operator.

In many experimental situations of interest, there is a preferred basis to select. For instance, if the dynamics is such that the system dephases to a given basis, the latter provides a natural choice. Which occurs, e.g., in the case of open quantum systems dynamics that are subject to environmental fluctuations. In other cases, it may make sense to choose the basis more arbitrarily (in advance), for instance, when analyzing a specific protocol or attempting to optimize it (see Ref. [61] for more details). Finally, the experimental setup might only allow for a measurement of one particular observable, in which case the chosen basis would correspond to the eigenbasis of said observable.

In what follows, we will analyze the classicality of a process based on the joint probability distributions obtained from sequential sharp measurements in a fixed basis $\{|x\rangle\}_{x=1}^{d}$-henceforth also called the classical, standard, or computational basis - with the action of a measurement with outcome $x$ on a state $\rho$ given by

$$
\rho \mapsto \mathcal{P}_{x}[\rho]:=|x\rangle\langle x|\rho| x\rangle\langle x| .
$$

See Fig. 1 for a graphical depiction.

This freedom in the considered measurements makes the property of classicality fundamentally contingent on the respective choice of measurement basis. However, this basis dependence is unsurprising and mirrored by coherence theory [2]. There, the existence of off-diagonal elements $\langle m|\rho| n\rangle$, i.e., coherences, depends on the choice of the basis a quantum state is represented in. As they are considered to be a fundamentally quantum property, it is a natural question to ask how coherences (with respect to the computational basis) and classicality of a process (with respect to the same basis) are interrelated. Importantly, while the existence of coherences cannot be determined by projective measurements in the computational basis alone, the prevalence of non-classical effects can be. Thus, as we shall see below, providing an operationally accessible

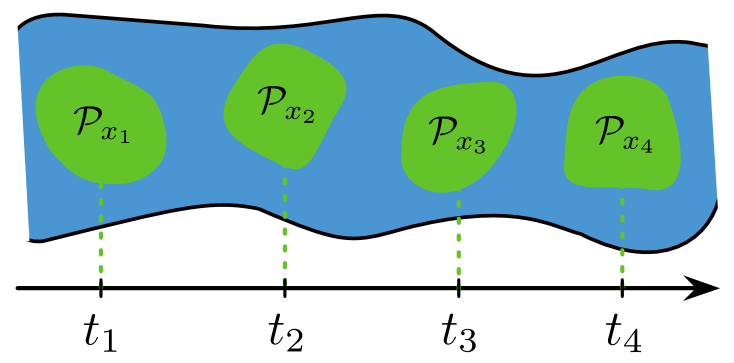

FIG. 1. Probing a process with projective measurements. At each time $t_{j}$, the process (depicted in blue) is probed by a projective measurement (depicted in green) with outcomes $x_{j}$, where each $x_{j}$ belongs to the same finite set $\mathcal{X}$. If the resulting family of probability distributions $\mathbb{P}_{n}$ (depicted are the cases $n \leq 4)$ satisfies the Kolmogorov consistency conditions, then not performing a measurement at a time $t_{j}$ cannot be distinguished from performing a measurement and averaging over the outcomes. In this case, this experiment cannot be distinguished from a classical one, even though the underlying evolution might be quantum mechanical.

notion of classicality allows one to link coherence (and, more generally, quantum correlations) in a quantitative manner to experimentally observable deviations from classical physics.

\section{Open (quantum) system dynamics and memory effects}

The definition of classicality we use (introduced in Ref. [34]) answers the question of whether or not there exists a classical stochastic process that can explain the multitime probabilities obtained by measuring a quantum system at given times in the computational basis. To make our analysis as general as possible, we consider the possibility that the measured system interacts with a surrounding environment, which can influence the resulting statistics. Explicitly, assuming that the system and environment in state $\eta$ are, together, closed and described by quantum mechanics, their joint dynamics between measurements is given by unitary evolution: $\mathcal{U}_{t_{j+1}, t_{j}}[\eta]=U_{t_{j+1}, t_{j}} \eta U_{t_{j+1}, t_{j}}^{\dagger}$. The resulting joint probability distributions read

$$
\begin{aligned}
\mathbb{P}_{n}\left(x_{n}, \ldots, x_{1}\right)= & \operatorname{tr}\left\{\left(\mathcal{P}_{x_{n}}^{s} \otimes \mathcal{I}^{e}\right) \circ \mathcal{U}_{t_{n}, t_{n-1}} \circ \cdots\right. \\
& \left.\cdots \circ\left(\mathcal{P}_{x_{1}}^{s} \otimes \mathcal{I}^{e}\right) \circ \mathcal{U}_{t_{1}, t_{0}}\left[\eta_{t_{0}}^{s e}\right]\right\}
\end{aligned}
$$

where $\eta_{t_{1}}^{s e}$ is the system-environment state at time $t_{1}, \mathcal{I}^{e}$ signifies the identity channel on the environment, $\mathcal{P}_{x_{j}}^{s}$ corresponds to a measurement on the system in the computational basis at time $t_{j}$ with outcome $x_{j}$, and $\circ$ denotes composition (see Fig. 2 for a graphical representation). Whenever there is no risk of confusion, we will drop the additional superscripts $s$ and $e$ throughout this paper. Naturally, the classicality of the family of joint probability distributions obtained via Eq. (11) crucially depends on the 


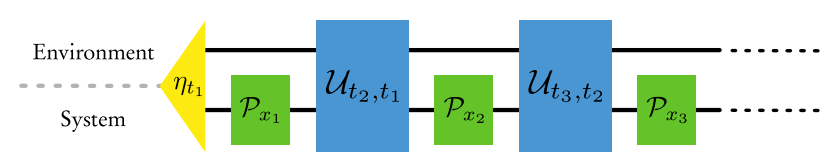

FIG. 2. General open quantum process. The state of the system at time $t_{1}$ is correlated with the environment (depicted by the yellow triangle representing the joint state). Measurements on the system (green boxes) are performed at times $t_{1}, t_{2}, \ldots$ In between, the system and the environment undergo a unitary evolution (blue boxes). The distinction between system and environment is given by the degrees of freedom that the experimenter controls (system) and those that remain inaccessible to experimental control (environment).

properties of the intermediate evolutions $\mathcal{U}_{t_{j+1}, t_{j}}$ and the initial state $\eta_{t_{0}}^{s e}$.

In general, such a multitime statistics displays memory effects; i.e., it is non-Markovian: At any point in time $t_{j}$, the future statistics not only depends on the measurement outcome $x_{j}$ at time $t_{j}$ but also on (potentially) all previous outcomes $x_{j-1}, \ldots, x_{1}$. Indeed, all information about future statistics at $t_{j}$ is contained in the joint state of system and environment, which depends upon the previous measurement outcomes. As this total state cannot be accessed by measurements on the system alone, this dependence on past measurements manifests itself as memory effects on the system level (see Sec. V for a detailed discussion).

However, under some specific circumstances, the influence of such memory effects on the multitime statistics can be neglected; this is essentially the case when the quantum regression formula (QRF) can be applied [29,62-64]. Under this assumption, the observed statistics can be understood in terms of dynamical propagators that act on the system alone, which, in turn, enables one to directly link the classicality of a process to the properties of said propagators in terms of coherence production and detection. The corresponding result has been obtained in Ref. [34], and we will reiterate and expand upon it in the next section. Subsequently, employing quantum combs - a powerful framework for the description of general, possibly non-Markovian open quantum processes-we characterize the set of quantum processes that can be described classically.

\section{COHERENCE AND CLASSICALITY}

In this section, we reiterate the main result of Ref. [34] on the connection between coherence and classicality for the memoryless case, generalizing it to the case of a divisible (but not necessarily semigroup [29,65,66]) dynamics. As mentioned above, such a direct connection may be established because memoryless processes can be understood in terms of propagators that are defined on the system alone, while this property fails to hold in the general, non-Markovian case.
After introducing an operational notion of Markovianity associated with the multitime statistics due to sequential measurements of a (nondegenerate) observable, we present a one-to-one connection between the nonclassicality of such statistics and the capability of the open system dynamics to generate and detect coherences with respect to the relevant basis. We also clarify the relation between the notion of Markovianity used in this paper and the QRF, which allows us to straightforwardly recover the main result of Ref. [34]. Finally, we lay out the subtleties that arise when generalizing the framework to allow for memory effects, motivating the main results of this work.

\section{A. One-to-one connection in the Markovian case}

Classically, a process is Markovian (i.e., memoryless) if, for any chosen time $t_{j}$, the future statistics only depend upon the outcome at time $t_{j}$ but not on any prior outcomes at $t_{j-1}, t_{j-2}, \cdots$; explicitly, a classical stochastic process is Markovian if its statistics satisfy

$$
\mathbb{P}\left(x_{j} \mid x_{j-1}, \ldots, x_{1}\right)=\mathbb{P}\left(x_{j} \mid x_{j-1}\right) \quad \forall j,
$$

where $\mathbb{P}\left(x_{j} \mid x_{j-1}, \ldots, x_{1}\right)$ is the conditional probability to obtain outcome $x_{j}$ at time $t_{j}$ given that outcomes $x_{j-1}, x_{j-2}, \ldots$ were measured at earlier times $t_{j-1}, t_{j-2}, \ldots$ [29]. Extending this definition to general (i.e., not necessarily classical) statistics and taking into account that, in practice, one only deals with systems probed at a finite number of times, we obtain the following definition of $K$ Markovianity:

Definition 2. Let $\mathcal{X}$ be a finite set. A process defined on a set of times $\mathcal{T}$, with $|\mathcal{T}|=K$ is called " $K$ Markovian" if it satisfies

$$
\mathbb{P}\left(x_{n} \mid x_{n-1}, \ldots, x_{1}\right)=\mathbb{P}\left(x_{n} \mid x_{n-1}\right) \quad \forall n \leq K,
$$

for all ordered tuples of times $t_{n} \geq \ldots \geq t_{1}$, with $t_{i} \in \mathcal{T}$, and $x_{i} \in \mathcal{X}$.

Just like our earlier definition of classicality and coherence, the absence of memory effects as defined in Definition 2 is basis dependent: A process that appears Markovian in one basis may appear non-Markovian when probed in a different one. While there exist basisindependent notions of Markovianity in the quantum case $[38,39,67-69]$, the basis-dependent one introduced here is best suited for the experimental situation we envision; as such, in what follows, we predominantly understand Markovianity with respect to measurements in the computational basis. We briefly return to the relation between this basis dependence and the basis-independent notion of Markovianity in Sec. V.

To establish a connection between nonclassicality of a Markovian process and the coherence properties of the underlying dynamics, we need to introduce the maps that characterize the dynamical evolution of the open system. 
To this end, assume that at an initial time $t_{0}$ (with $t_{0} \leq t_{1}$ ), the system and the environment are in a product state $\eta_{t_{0}}^{s e}=$ $\rho_{t_{0}} \otimes \sigma_{t_{0}}$ (for some fixed environment state $\sigma_{t_{0}}$ ), so we can define the CPTP dynamical maps $\left\{\Lambda_{t_{j}, t_{0}}\right\}$ of the open system evolution between the initial time and the measurement times $t_{j}[29,70]$ as

$$
\rho_{t_{j}}=\Lambda_{t_{j}, t_{0}}\left[\rho_{t_{0}}\right]=\operatorname{tr}_{e}\left[U_{t_{j}, t_{0}}\left(\rho_{t_{0}} \otimes \sigma_{t_{0}}\right) U_{t_{j}, t_{0}}^{\dagger}\right]
$$

where $\operatorname{tr}_{e}$ denotes the trace over the environmental degrees of freedom. Additionally, let us also assume that the dynamics is divisible [71]; i.e, we can define the corresponding propagators $\left\{\Lambda_{t_{k}, t_{j}}\right\}$ between any two times via the composition rule

$$
\Lambda_{t_{k}, t_{0}}=\Lambda_{t_{k}, t_{j}} \circ \Lambda_{t_{j}, t_{0}} \quad \forall t_{k} \geq t_{j} \geq t_{0},
$$

and they satisfy the composition law $\Lambda_{t_{\ell}, t_{j}}=\Lambda_{t_{\ell}, t_{k}} \circ \Lambda_{t_{k}, t_{j}}$ for all times $t_{\ell} \geq t_{k} \geq t_{j}$. Under these assumptions, it is natural to ask what conditions the propagators $\left\{\Lambda_{t_{k}, t_{j}}\right\}$ must satisfy in order for the resulting statistics to be classical. However, Eq. (15) does not yet tell us how to obtain multitime statistics [72].

The relation we seek is provided by the QRF, which, for example, holds in the weak coupling and the singular coupling limits [73] and constitutes a relation between the definition of Markovian processes given by Definition 2 and the corresponding open system dynamics (see also Ref. [69] for an extensive discussion of the QRF and its generalizations). For the case of rank-1 projective measurements (in the computational basis), the QRF states that the multitime probability distributions in Eq. (11) can be equivalently expressed by

$$
\begin{aligned}
& \mathbb{P}_{n}\left(x_{n}, \ldots, x_{1}\right) \\
& \quad=\operatorname{tr}\left\{\mathcal{P}_{x_{n}} \circ \Lambda_{t_{n}, t_{n-1}} \circ \cdots \circ \Lambda_{t_{2}, t_{1}} \circ \mathcal{P}_{x_{1}} \circ \Lambda_{t_{1}, t_{0}}\left[\rho_{t_{0}}\right]\right\} .
\end{aligned}
$$

Importantly, this expression means that the full multitime statistics can be obtained by means of maps that are independent of the respective previous measurement outcomes and that act on the system alone (see Fig. 3 for a graphical representation).

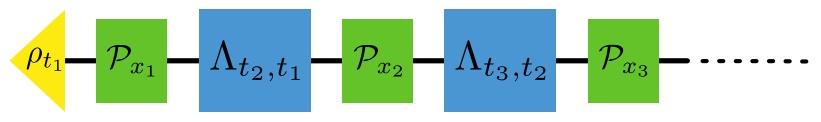

FIG. 3. Markovian process. For a Markovian process, the system dynamics in between intermediate times (depicted as the blue boxes) can be modeled by maps $\Lambda_{t_{j+1}, t_{j}}$ that do not depend on previous outcomes (i.e., there is no memory). The measurement statistics are obtained by measuring in the classical basis at times $t_{1}, t_{2}, t_{3}, \ldots$ (depicted in green); before the first measurement, the system is in the state $\rho_{t_{1}}$ (depicted in yellow).
It is straightforward to see that satisfaction of the QRF [see Eq. (16)] implies Markovian statistics in the sense of Eq. (13), and in particular, we have the identities

$$
\begin{gathered}
\left\langle x_{k}\left|\Lambda_{t_{k}, t_{j}}\left[\left|x_{j}\right\rangle\left\langle x_{j}\right|\right]\right| x_{k}\right\rangle=\mathbb{P}\left(x_{k} \mid x_{j}\right) \quad \forall j \geq 1, \\
\text { and }\left\langle x_{1}\left|\Lambda_{t_{1}, t_{0}}\left[\rho_{t_{0}}\right]\right| x_{1}\right\rangle=\mathbb{P}\left(x_{1}\right) .
\end{gathered}
$$

In other words, the action of the propagators on the populations (i.e., the diagonal terms of $\rho_{t_{j}}$, the state of the system at $t_{j}$ ) can be identified with the conditional probabilities between any two times. Crucially, this generally is not the case, and it breaks down in situations where the QRF cannot be applied [74].

More generally, even if the QRF applies, the composition rule on the level of propagators does not imply a composition rule on the level of the resulting measurement statistics; i.e., for a divisible process that satisfies the QRF, we generally have

$$
\sum_{x_{k}} \mathbb{P}\left(x_{\ell} \mid x_{k}\right) \mathbb{P}\left(x_{k} \mid x_{j}\right) \neq \mathbb{P}\left(x_{\ell} \mid x_{j}\right),
$$

which captures the deviation of quantum Markovian processes from classical ones. As mentioned previously, in order for the resulting process to be classical, not performing a measurement must be indistinguishable from performing a measurement and averaging over all possible outcomes. Put differently, for an observer that can only perform measurements in a fixed basis, the process is classical if it cannot detect the invasiveness of measurements in said basis.

A measurement at time $t_{j}$ in the fixed basis where the measurement outcomes are averaged over can be represented by the completely dephasing map

$$
\Delta[\rho]=\sum_{x_{j}} \mathcal{P}_{x_{j}}[\rho]=\sum_{x_{j}}\left\langle x_{j}|\rho| x_{j}\right\rangle\left|x_{j}\right\rangle\left\langle x_{j}\right| .
$$

The natural property of the propagators to examine in relation to classicality is thus that for all $t_{j}$,

$$
\begin{aligned}
& \Delta_{j+1} \circ \Lambda_{t_{j+1}, t_{j}} \circ \Delta_{j} \circ \Lambda_{t_{j}, t_{j-1}} \circ \Delta_{j-1} \\
& =\Delta_{j+1} \circ \Lambda_{t_{j+1}, t_{j}} \circ \mathcal{I}_{j} \circ \Lambda_{t_{j}, t_{j-1}} \circ \Delta_{j-1} \\
& =\Delta_{j+1} \circ \Lambda_{t_{j+1}, t_{j-1}} \circ \Delta_{j-1},
\end{aligned}
$$

where $\mathcal{I}_{j}$ and $\Lambda_{j}$ are the identity map and the completely dephasing map at time $t_{j}$, respectively (see Fig. 4 for a graphical representation). In the last line of Eq. (21), we used the composition law $\Lambda_{t_{j+1}, t_{j-1}}=\Lambda_{t_{j+1}, t_{j}} \circ \Lambda_{t_{j}, t_{j-1}}$. Equation (21) is, e.g., satisfied if none of the maps $\left\{\Lambda_{t_{j+1}, t_{j}}\right\}$ create coherences. More generally, each of the maps in Eq. (21) can, in principle, create coherences, as long as these coherences cannot be detected at the next time 


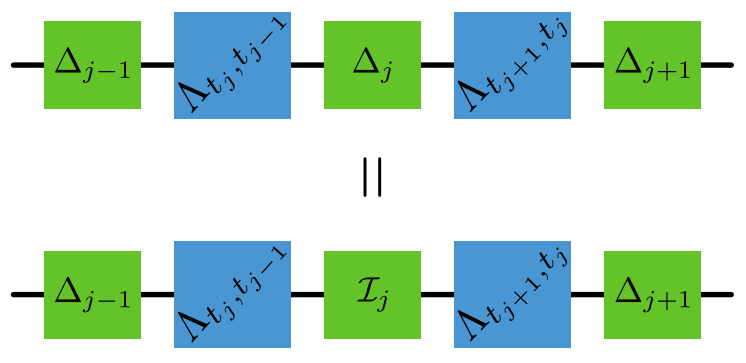

FIG. 4. NCGD dynamics. If the process is NCGD, then for a classical observer, "doing nothing" (i.e., performing the map $\mathcal{I}$ ) cannot be distinguished from a measurement in the classical basis and averaging over the outcomes (i.e., performing the map $\Delta$ ) at any point in time.

by means of measurements in the classical basis. Therefore, such a collection of maps satisfying Eq. (21) are called noncoherence-generating-and-detecting (NCGD) maps [34]. The precise connection between NCGD and classicality is expressed by the following theorem:

Theorem 1. Let $\left\{\mathbb{P}_{n}\left(x_{n}, \ldots, x_{1}\right)\right\}_{n \leq K}$ be a $K$-Markovian process (Definition 2). Then, the process is also $K$ classical (Definition 1) if and only if there exists a system state $\rho_{t_{0}}$ (at a time $t_{0} \leq t_{1}$ ) that is diagonal in the computational basis $\{|x\rangle\}_{x \in \mathcal{X}}$ and a set of propagators $\left\{\Lambda_{t_{j}, t_{j-1}}\right\}_{j=1, \ldots, K}$ that are NCGD with respect to $\{|x\rangle\}_{x \in \mathcal{X}}$, such that $\rho_{t_{0}}$ and $\left\{\Lambda_{t_{j}, t_{j-1}}\right\}_{j=1, \ldots, K}$ yield $\left\{\mathbb{P}_{n}\left(x_{n}, \ldots, x_{1}\right)\right\}_{n \leq K}$ via Eq. (16).

Proof.-We first show that if a Markovian process can be reproduced by means of NCGD propagators $\left\{\Lambda_{t_{j+1}, t_{j}}\right\}$ and an initial diagonal state (both properties with respect to the computational basis), then it yields classical statistics. If the statistics is Markovian, then it follows from Eq. (13) that the joint probability distribution on any set of times $t_{n} \geq \ldots \geq t_{1}$, with $t_{i} \in \mathcal{T}$, is given by

$$
\mathbb{P}_{n}\left(x_{n}, \ldots, x_{1}\right)=\mathbb{P}\left(x_{n} \mid x_{n-1}\right) \cdots \mathbb{P}\left(x_{2} \mid x_{1}\right) \mathbb{P}\left(x_{1}\right) .
$$

As the process can, by assumption, be reproduced by the maps $\left\{\Lambda_{t_{j}, t_{j-1}}\right\}$ via Eq. (16), then for any time $t_{j}$, for $j>1$, we have

$$
\begin{aligned}
& \sum_{x_{j}} \mathbb{P}\left(x_{j+1} \mid x_{j}\right) \mathbb{P}\left(x_{j} \mid x_{j-1}\right) \\
& =\sum_{x_{j}} \operatorname{tr}\left\{\mathcal{P}_{x_{j+1}} \circ \Lambda_{t_{j+1}, t_{j}}\left[\Pi_{x_{j}}\right]\right\} \operatorname{tr}\left\{\mathcal{P}_{x_{j}} \circ \Lambda_{t_{j}, t_{j-1}}\left[\Pi_{x_{j-1}}\right]\right\} \\
& =\operatorname{tr}\left\{\mathcal{P}_{x_{j+1}} \circ \Lambda_{t_{j+1}, t_{j}} \circ \Delta_{j} \circ \Lambda_{t_{j}, t_{j-1}}\left[\Pi_{x_{j-1}}\right]\right\} \\
& =\operatorname{tr}\left\{\mathcal{P}_{x_{j+1}} \circ \Lambda_{t_{j+1}, t_{j-1}}\left[\Pi_{x_{j-1}}\right]\right\},
\end{aligned}
$$

where we have set $\Pi_{x_{j}}=\left|x_{j}\right\rangle\left\langle x_{j}\right|$ and the NCGD property was used in the last line. This equation implies

$$
\sum_{x_{j}} \mathbb{P}\left(x_{j+1} \mid x_{j}\right) \mathbb{P}\left(x_{j} \mid x_{j-1}\right)=\mathbb{P}\left(x_{j+1} \mid x_{j-1}\right) .
$$

Moreover, the (initial) diagonal state $\rho_{t_{0}}$ guarantees that we have

$$
\sum_{x_{1}} \mathbb{P}\left(x_{2}, x_{1}\right)=\mathbb{P}\left(x_{2}\right) .
$$

As a consequence of these two previous relations, the family of joint probability distributions computed via Eq. (22) satisfies Kolmogorov conditions and is thus classical.

Conversely, if the process is classical and Markovian, Eq. (24) holds. We can then define the maps

$$
\tilde{\Lambda}_{t_{j+1}, t_{j}}\left[\left|x_{j}\right\rangle\left\langle y_{j}\right|\right]=\delta_{x_{j} y_{j}} \sum_{x_{j+1}} \mathbb{P}\left(x_{j+1} \mid x_{j}\right) \Pi_{x_{j+1}},
$$

and the initial diagonal state

$$
\tilde{\rho}_{t_{0}}=\sum_{x_{1}} \mathbb{P}\left(x_{1}\right) \Pi_{x_{1}}
$$

which also means that we identify the initial time as the time of the first measurement, $t_{1}=t_{0}$. The set of maps $\left\{\tilde{\Lambda}_{t_{j+1}, t_{j}}\right\}$ defined in this way, in conjunction with $\tilde{\rho}_{t_{0}}$, reproduces the correct statistics via Eq. (16). As they are diagonal in the computational basis for any pair of times $t_{j}$ and $t_{j+1}$, they form a NCGD set.

Crucially, the connection between classicality and NCGD dynamics is one-to-one: If the obtained Markovian statistics cannot be reproduced by a set of maps that are NCGD, then the process is nonclassical. Before discussing classicality in the presence of memory effects below, it is worth discussing the intuitive meaning of this theorem and NCGD dynamics, in particular.

If the process at hand is Markovian and classical, the maps $\left\{\tilde{\Lambda}_{t_{j+1}, t_{j}}\right\}$ (as well as the initial state $\tilde{\rho}_{t_{0}}$ ) introduced in the proof of Theorem 1 define an artificial reduced dynamics of the system, whose propagators correctly reproduce all joint probability distributions for measurements in the (fixed) classical basis via Eq. (16). Note that the actual propagators of the dynamics [i.e., those fixed by the unitary evolution in Eq. (11) via Eqs. (14) and (15)] might differ from the maps $\tilde{\Lambda}_{t_{j+1}, t_{j}}$ above (and $\tilde{\rho}_{t_{0}}$ might differ from the actual initial state $\rho_{t_{0}}$ ); indeed, the fact that they do not coincide is simply a manifestation of the basis dependence of the (sequential) measurement scheme we are focusing on here.

Crucially, a composition rule on the level of the actual propagators does not imply a composition rule on the level of the propagators of the populations. This implication only holds if the propagators of the dynamics are NCGD, and the resulting statistics can be computed via Eq. (16), in which case Eq. (21) results in 


$$
\tilde{\Lambda}_{t_{j+1}, t_{j-1}}=\tilde{\Lambda}_{t_{j+1}, t_{j}} \circ \tilde{\Lambda}_{t_{j}, t_{j-1}} \quad \forall t_{j},
$$

with

$$
\tilde{\Lambda}_{t_{k}, t_{j}}\left[\left|x_{j}\right\rangle\left\langle y_{j}\right|\right]=\delta_{x_{j} y_{j}}\left\langle x_{k}\left|\Lambda_{t_{k}, t_{j}}\left[\left|x_{j}\right\rangle\left\langle x_{j}\right|\right]\right| x_{k}\right\rangle \Pi_{x_{j}}
$$

[see Eqs. (17) and (26)]. These reduced propagators still produce the correct populations, which are the only relevant part for the considered statistics, and set all coherences to zero. This composition law is then-as already seen in Eq. (24) - equivalent to the well-known classical Chapman-Kolmogorov equations

$$
\sum_{x_{j}} \mathbb{P}\left(x_{j+1} \mid x_{j}\right) \mathbb{P}\left(x_{j} \mid x_{j-1}\right)=\mathbb{P}\left(x_{j+1} \mid x_{j-1}\right),
$$

which hold for classical Markovian processes: If the measurement statistics of a Markovian process can be reproduced by a set of NCGD maps $\left\{\Lambda_{t_{j}, t_{j-1}}\right\}$, then it can also be reproduced by the set of maps $\left\{\tilde{\Lambda}_{t_{j}, t_{j-1}}\right\}$, which act nontrivially on only the populations of the computational basis and satisfy a composition law; thus, the process is classical.

Conversely, if the classical composition rule of Eq. (30) holds for a Markovian process, then there exists a set $\left\{\tilde{\Lambda}_{t_{j+1}, t_{j}}\right\}$ of propagators [e.g., those defined in Eq. (26)] that are NCGD and correctly reproduce all joint probability distributions for measurements in the (fixed) classical basis.

Theorem 1 is a generalization of the main result of Ref. [34] in two ways. First, it does not impose any restriction on the propagators of the underlying quantum evolution, while in Ref. [34], these were required to form a semigroup, i.e., $\Lambda_{t_{j+1}, t_{j}}=e^{\mathcal{L}\left(t_{j+1}-t_{j}\right)}$, for some Lindbladian $\mathcal{L}[65,66]$.

Second, the definition of Markovianity used here coincides with the standard definition of classical stochastic processes, whereas in Ref. [34], a definition based on Eq. (16) (for semigroups) was used. Consequently, while the maps $\left\{\Lambda_{t_{j+1}, t_{j}}\right\}$ cannot be fully probed by measurements in the computational basis alone, the requirement of Eq. (30) can be tested by simply performing sequences of measurements in the classical basis at the relevant times, thus making our theorem fully operational. However, this feature comes at the cost of dealing with propagators $\left\{\tilde{\Lambda}_{t_{j+1}, t_{j}}\right\}$, which might not correspond to those of the actual reduced dynamics.

On the other hand, as we show in the Appendix A, a oneto-one correspondence between the dynamical propagators $\Lambda_{t_{j+1}, t_{j}}$ and the nonclassicality of the multitime statistics can also be established in the general (nonsemigroup) divisible case, when the QRF applies, provided that one assumes a proper invertibility condition on the restriction of the dynamical maps to the populations of the computational basis. Indeed, this correspondence also allows one to recover, in a straightforward way, the main result of Ref. [34] as a corollary by further imposing the semigroup composition law.

Importantly, Theorem 1 characterizes the connection between coherences and the classicality of a Markovian process. While it is not necessary that the underlying propagators create no coherences in order for a Markovian process to be classical, it is necessary and sufficient that coherences-should they be created—cannot be detected at a later point in time by means of measurements in the computational basis. Put differently, the propagators must be such that a classical observer could not decide whether, at any point in time, an identity map or a completely dephasing map was performed (which is depicted in Fig. 4). This requirement is exactly encapsulated in the NCGD property of the propagators.

\section{B. Coherence in the non-Markovian case: Preliminary analysis}

The above connection between quantum coherence and nonclassicality fails to hold in the non-Markovian case. On the one hand, in this case, propagators between two times are no longer sufficient to fully characterize the multitime statistics [75]. On the other hand, even if the state of the system is diagonal in the computational basis at all times, dephasing can still be invasive due to correlations with the environment, breaking the connection between coherences and the classicality of statistics. We will discuss the former problem in the subsequent sections. Using an open system model from Refs. [68,78,79], an explicit ante litteram example of the latter case has already been provided in Ref. [34] (note, also, a similar investigation in Ref. [80]), albeit not with an emphasis on the lack of coherence in the system state at all times (even in between the measurements). Here, we reiterate this example, focusing on the absence of coherences in the state of the system. The details of this discussion can be found in Appendixes B and C. A simpler, although noncontinuous, example for a nonMarkovian process that yields nonclassical statistics but never displays coherences in the system state is provided in Appendix D.

Example 1. Let the system of interest $s$ consist of a qubit described by $\rho_{s}(t)$ which is coupled to a continuous degree of freedom $p$ of the environment. The global dynamics of the system and environment is governed by the unitary evolution $U_{t_{j}, t_{i}}$, acting as

$$
U_{t_{j}, t_{i}}|\ell, p\rangle=e^{i \phi_{\ell} p\left(t_{j}-t_{i}\right)}|\ell, p\rangle,
$$

where $\{|\ell\rangle\}_{\ell=0,1}$ is the eigenbasis of the system Pauli operator $\hat{\sigma}_{z}$ and $\phi_{\ell}=(-1)^{\ell}$. The initial systemenvironment state is assumed to be of product form $\eta(0)=\rho_{s}(0) \otimes\left|\varphi^{e}\right\rangle\left\langle\varphi^{e}\right|, \quad$ with $\left|\varphi^{e}\right\rangle=\int_{-\infty}^{\infty} d p f(p)|p\rangle$, where $f(p)$ satisfies the normalization condition $\int_{-\infty}^{\infty} d p|f(p)|^{2}=1$. By defining 


$$
k(t):=\int_{-\infty}^{\infty} d p|f(p)|^{2} e^{2 i p t},
$$

it is straightforward to show that, expressed in the eigenbasis of $\hat{\sigma}_{z}$, the free open evolution of the state of the system (i.e., without intermediate measurements) is given by

$$
\rho_{s}(t)=\left(\begin{array}{cc}
\rho_{00} & k(t) \rho_{01} \\
k^{*}(t) \rho_{10} & \rho_{11}
\end{array}\right),
$$

where $\rho_{m n}:=\left\langle m\left|\rho_{s}(0)\right| n\right\rangle$.

If $\rho_{s}(0)$ is initialized in a convex mixture of the eigenvectors $\{| \pm\rangle=(|0\rangle \pm|1\rangle) / \sqrt{2}\}$ of the $\hat{\sigma}_{x}$ operator, i.e., $\rho_{s}(0)=\alpha|+\rangle\langle+|+(1-\alpha)|-\rangle\langle-|$, then

$$
\begin{aligned}
\rho_{s}(t)= & \frac{1}{2}\left(\begin{array}{cc}
1 & k(t)(2 \alpha-1) \\
k^{*}(t)(2 \alpha-1) & 1
\end{array}\right) \\
= & \frac{1}{2}\{|+\rangle\langle+|[1+(2 \alpha-1) \operatorname{Re}(k(t))] \\
& -|+\rangle\langle-|(2 \alpha-1) \operatorname{Im}(k(t)) \\
& +|-\rangle\langle+|(2 \alpha-1) \operatorname{Im}(k(t)) \\
& +|-\rangle\langle-|[1-(2 \alpha-1) \operatorname{Re}(k(t))]\} ;
\end{aligned}
$$

i.e., no coherence with respect to $\hat{\sigma}_{x}$ will be generated if $k(t)$ is a real function of time (as noted in Ref. [34]); this is, e.g., the case if $f(p)$ corresponds to a Lorentzian distribution centered around zero,

$$
|f(p)|^{2}=\frac{\Gamma}{\pi\left(\Gamma^{2}+p^{2}\right)} \mapsto k(t)=e^{-2 \Gamma|t|} .
$$

A priori, the fact that there are no $\hat{\sigma}_{x}$ coherences created in the free evolution does not mean that none are created if the system is probed at intermediate times. However, here, no $\hat{\sigma}_{x}$ coherence is generated even when we take into account how the measurements modify the system's state. Specifically, immediately after a measurement in the $\hat{\sigma}_{x}$ basis is performed at time $t_{1}$ (yielding outcome \pm ), the total system-environment state is of product form

$$
\eta^{( \pm)}\left(t_{1}\right)=| \pm\rangle\langle \pm| \otimes \xi^{( \pm)}\left(t_{1}\right),
$$

where $\xi^{( \pm)}\left(t_{1}\right)$ is a state of the environment that depends on the measurement outcome. As we show in Appendix B, any state of the system evolved from the postmeasurement state of Eq. (36) according to the described dynamics remains diagonal in the $\{| \pm\rangle\}$ basis; this also holds true for the state of the system after any sequence of such measurements. Together with the fact that the statistics resulting from measurements in the $\{| \pm\rangle\}$ basis is nonclassical (i.e., it does not satisfy Kolmogorov conditions, as has been shown in Ref. [34]), this constitutes an example of a nonclassical process without any coherence with respect to the measured observable ever being generated. Evidently, this behavior is only possible since the chosen example is non-Markovian.

Unlike in the Markovian case, where the absence of coherences trivially leads to classical statistics, when memory effects are present, it is the coherences of the system state as well as the nonclassical correlations between the system and its environment that can lead to nonclassical behavior-in a way that will be specified in the following. Intuitively, while the completely dephasing map leaves the system unchanged if no coherences are created, it does not necessarily leave the overall systemenvironment state invariant. In detail, in general, we can have $\Delta\left[\rho_{t_{j}}^{s}\right]=\mathcal{I}\left[\rho_{t_{j}}^{s}\right] \forall t_{j}$, without implying $\Delta \otimes \mathcal{I}^{e}\left[\eta_{t_{j}}^{s e}\right]=$ $\mathcal{I}\left[\eta_{t_{j}}^{s e}\right] \quad \forall t_{j}$. As we will see, the latter property is sufficient, but not necessary, for the satisfaction of the Kolmogorov conditions. First, though, in order to be able to go beyond the investigation of Markovian processes and extend the existing connection between classicality and coherences, it is important to introduce quantum combs-a suitable framework to describe general quantum processes $[37,38]$.

\section{NON-MARKOVIAN CLASSICAL PROCESSES}

The previous example illustrates the subtle relation between coherence and classicality in the case of open quantum processes with memory. There, although no coherence is ever generated on the level of the system with respect to the chosen measurement basis, the systemenvironment correlations built up throughout the dynamics lead to nonclassical statistics. To develop a more in-depth understanding of the interplay between coherences and classical phenomena, we require a suitable operational framework for approaching such scenarios. We can then employ this framework to comprehensively characterize all quantum processes that display classical statistics.

\section{A. Classicality and processes with memory}

The necessity of such a novel framework for the description of quantum processes that display memory effects stems from the breakdown of their modeling in terms of propagators that could be used in the Markovian case; this can already be seen for classical stochastic processes. Here, a joint probability distribution $\mathbb{P}_{K}\left(x_{K}, \ldots, x_{1}\right)$ fully describes a $K$ process. This probability distribution can equivalently be represented in terms of multitime conditional probabilities as

$$
\begin{aligned}
& \mathbb{P}_{K}\left(x_{K}, \ldots, x_{1}\right) \\
& \quad=\mathbb{P}_{K}\left(x_{K} \mid x_{K-1}, \ldots, x_{1}\right) \cdots \mathbb{P}_{2}\left(x_{2} \mid x_{1}\right) \mathbb{P}_{1}\left(x_{1}\right) .
\end{aligned}
$$

Importantly, all of the above conditional probabilities generally depend upon all preceding measurement results, in contrast to the Markovian case where they only depend on the most recent outcome. Consequently, two-point transition probabilities of the form $\mathbb{P}\left(x_{j} \mid x_{j-1}\right)$ are not sufficient, in general, to build up all joint probability distributions and thus 
do not completely describe the process. Similarly, two-time propagators $\left\{\Lambda_{t_{j}, t_{j-1}}\right\}$ are generally not sufficient to compute multitime joint probabilities in the quantum case and therefore fail to fully characterize the process $[74,81]$.

For classical statistics, the joint probability distribution $\mathbb{P}_{K}\left(x_{K}, \ldots, x_{1}\right)$ contains all information about the $K$ process since all distributions for fewer times, as well as all conditional probabilities, can be derived once $\mathbb{P}_{K}$ is known. In exactly the same way, a general quantum $K$ process is fully characterized by the joint probabilities for all possible sequences of $K$ measurements (at times $t_{1}, \ldots, t_{K}$ ), including nonprojective and nonorthogonal ones.

As discussed in the previous section, if the complete system-environment dynamics is known, then all joint probability distributions (on times $\left\{t_{j}\right\}_{j=1}^{n}$ ) obtained from sequential measurements of the system can be computed via

$$
\begin{aligned}
& \mathbb{P}_{n}\left(x_{n}, \ldots, x_{1}\right) \\
& \quad=\operatorname{tr}\left\{\left(\mathcal{P}_{x_{n}} \otimes \mathcal{I}^{e}\right) \circ \mathcal{U}_{t_{n}, t_{n-1}} \circ \cdots \circ\left(\mathcal{P}_{x_{1}} \otimes \mathcal{I}^{e}\right)\left[\eta_{t_{1}}^{s e}\right]\right\} .
\end{aligned}
$$

Here, $\left\{\mathcal{P}_{x_{j}}\right\}$ corresponds to a set of projective measurements in the computational basis, but evidently, the same relation can also be used to obtain the correct probabilities when using different probing instruments, e.g., instruments that measure sharply in a different basis or those that perform generalized measurements. More formally, an instrument $\mathcal{J}_{k}=\left\{\mathcal{M}_{x_{k}}\right\}$ (at time $t_{k}$ ) is a collection of CP maps that add up to a CPTP map [60]. For instance, the instrument corresponding to a measurement in the computational basis is given by $\mathcal{J}_{k}=\left\{\mathcal{P}_{x_{k}}\right\}$, and all of its elements add up to the CPTP map $\sum_{x_{k}} \mathcal{P}_{x_{k}}=\Delta_{k}$. Intuitively, each outcome of an instrument corresponds to one of its constituent CP maps, which, in turn, describes how the state of the system changes upon the realization of said measurement outcome. Thus, the probability to obtain the sequence of outcomes $x_{1}, \ldots, x_{K}$, given that the instruments $\mathcal{J}_{1}, \ldots, \mathcal{J}_{K}$ were used to probe the system, is given by

$$
\begin{aligned}
& \mathbb{P}_{K}\left(x_{K}, \ldots, x_{1} \mid \mathcal{J}_{K}, \ldots, \mathcal{J}_{1}\right) \\
& \quad=\operatorname{tr}\left\{\left(\mathcal{M}_{x_{K}} \otimes \mathcal{I}^{e}\right) \circ \mathcal{U}_{t_{K}, t_{K-1}} \circ \cdots \circ\left(\mathcal{M}_{x_{1}} \otimes \mathcal{I}^{e}\right)\left[\eta_{t_{1}}^{s e}\right]\right\} \\
& \quad=: \mathcal{C}_{K}\left[\mathcal{M}_{x_{K}}, \ldots, \mathcal{M}_{x_{1}}\right]
\end{aligned}
$$

indeed, the joint probability distribution for any subset of ordered times $t_{n} \geq \ldots \geq t_{1}$, with $n<K$, can be obtained from the formula above by replacing $\mathcal{M}_{x_{j}}$ with the identity operator, in correspondence with the times $t_{j}$ not contained in the subset of interest.

In what follows, whenever we drop the explicit instrument labels, it is understood that the probabilities are the result of a measurement in the computational basis at each time. The multilinear functional $\mathcal{C}_{K}$ introduced above is a special case [82] of a quantum comb [37,51], which provides a natural generalization to the concept of quantum

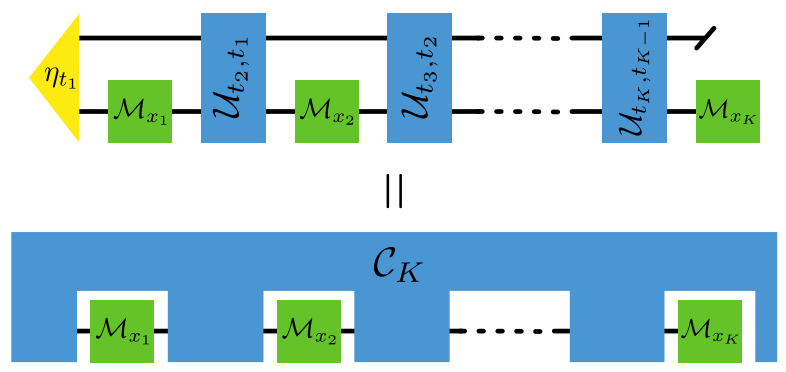

FIG. 5. Comb of a general open quantum evolution. The probabilities characterizing a quantum process can be computed via as the action of a comb $\mathcal{C}_{K}$ on the sequence of $\mathrm{CP}$ maps $\left\{\mathcal{M}_{x_{j}}\right\}$ that correspond to the respective measurement outcomes.

channels that, by construction, allows for the inclusion of memory effects $[38,39,83,84]$ (see Fig. 5 for a graphical representation). It maps any sequence of possible experimental transformations enacted on the system to the corresponding joint probability of their occurrence. In this sense, $\mathcal{C}_{K}$ plays exactly the same role that the joint probability distribution $\mathbb{P}_{K}$ plays in the classical setting and thus allows one to decide on the classicality of the resulting statistics. For example, for the completely memoryless case, i.e, the case of Markovianity with respect to measurements in any basis, the evolution between any two points in time is described solely by a sequence of independent CPTP maps $\left\{\Lambda_{t_{j}, t_{j-1}}\right\}$ that act on the system alone $[39,85]$, and we have

$$
\begin{aligned}
& \mathcal{C}_{K}^{\text {Markov }}\left[\mathcal{M}_{x_{K}}, \ldots, \mathcal{M}_{x_{1}}\right] \\
& \quad=\operatorname{tr}\left\{\mathcal{M}_{x_{K}} \circ \Lambda_{t_{K}, t_{K-1}} \circ \cdots \circ \mathcal{M}_{x_{2}} \circ \Lambda_{t_{2}, t_{1}} \circ \mathcal{M}_{x_{1}}\left[\rho_{t_{1}}\right]\right\} .
\end{aligned}
$$

In general, however, the comb of a $K$ process does not split into independent portions of evolution between times, in contrast to the above. Thus, when analyzing the relation between coherence and classicality in the presence of memory, instead of investigating the properties of individual CPTP maps (or collections of them), one must consider those of the multitime comb $\mathcal{C}_{K}$.

The $\operatorname{comb} \mathcal{C}_{K}$ is an operationally well-defined object that can-just like the joint probability distribution $\mathbb{P}_{K}$-be obtained by means of probing measurements on the system alone, through a generalized tomographic scheme $[38,86]$. Specifically, for its reconstruction, it is not necessary to explicitly know the system-environment dynamics: The comb does not contain direct information about the environment but solely that of its influence on the multitime statistics observed from measurements on the system. As such, it encapsulates all that is out of the control of the experimenter and thereby clearly separates the underlying process at hand from what can be controlled (i.e., the experimental interventions). An explicit example of the comb formalism is provided in Appendix C, where we rephrase Example 1 in terms of the comb description. 


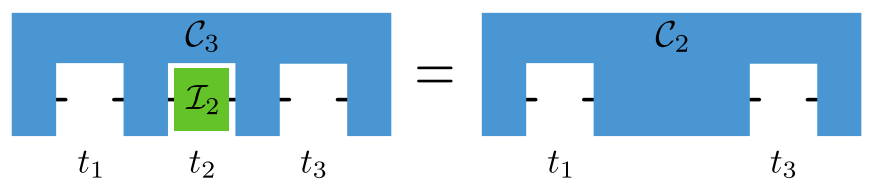

FIG. 6. Generalized marginalization for combs. Letting a comb defined on times $\mathcal{T}$ act on identity maps at a set of times $\mathcal{T} \backslash \mathcal{T}^{\prime}$ (i.e., the set of times in $\mathcal{T}$ but not in $\mathcal{T}^{\prime}$ ) yields the correct comb on $\mathcal{T}^{\prime}$. Depicted is the situation for $\mathcal{T}=\left\{t_{1}, t_{2}, t_{3}\right\}$ and $\mathcal{T}^{\prime}=\left\{t_{1}, t_{3}\right\}$

Crucially, the comb framework allows us to consider what it means for a stochastic process with memory to be classical, thereby permitting an extension of the results of Ref. [34] to the non-Markovian case: Given the $\operatorname{comb} \mathcal{C}_{K}$ of a process on times in $\mathcal{T}$, all combs correctly describing the process on fewer times $\mathcal{T}^{\prime} \subseteq \mathcal{T}$ can be deduced by letting $\mathcal{C}_{K}$ act on the identity map at the appropriate superfluous times $[38,56]$. For example, we have (see also Fig. 6)

$$
\begin{aligned}
& \mathcal{C}_{K-1}\left[\mathcal{M}_{x_{K}}, \ldots, \mathcal{M}_{x_{j+1}}, \mathcal{M}_{x_{j-1}}, \ldots, \mathcal{M}_{x_{1}}\right] \\
& \quad=\mathcal{C}_{K}\left[\mathcal{M}_{x_{K}}, \ldots, \mathcal{M}_{x_{j+1}}, \mathcal{I}_{j}, \mathcal{M}_{x_{j-1}}, \ldots, \mathcal{M}_{x_{1}}\right]
\end{aligned}
$$

As we have discussed in the previous sections, classicality of a process means that the action of the completely dephasing map cannot be distinguished (by means of measurements in the classical basis) from not performing an operation. With the method of "generalized marginalization" given by Eq. (41), we obtain the following characterization of classical combs:

Theorem 2. ( $K$-classical quantum combs) A comb $\mathcal{C}_{K}$ on times $\mathcal{T}$, with $|\mathcal{T}|=K$, yields a $K$-classical process iff it satisfies

$$
\begin{aligned}
\mathcal{C}_{K} & {\left[\underset{t_{j} \in \mathcal{T}^{\prime}}{\otimes} \mathcal{I}_{j}, \underset{t_{k} \in \mathcal{T} \backslash \mathcal{T}^{\prime}}{\otimes} \mathcal{P}_{x_{k}}\right] } \\
& =\mathcal{C}_{K}\left[{\underset{t}{t_{j} \in \mathcal{T}^{\prime}}}_{\boldsymbol{T}_{j}} \Delta_{j}, \underset{t_{k} \in \mathcal{T} \backslash \mathcal{T}^{\prime}}{\otimes} \mathcal{P}_{x_{k}}\right],
\end{aligned}
$$

for all subsets $\mathcal{T}^{\prime} \subseteq \mathcal{T}$ and all possible sequences of outcomes on $\mathcal{T} \backslash \mathcal{T}^{\prime}$.

In a slight abuse of notation, here, the argument $\otimes_{t_{j} \in \mathcal{T}^{\prime}}$ $a_{j}, \bigotimes_{t_{k} \in \mathcal{T} \backslash \mathcal{T}^{\prime}} b_{x_{k}}$ of the comb $\mathcal{C}_{K}$ signifies that it acts on the maps $a_{j}$ at times $t_{j} \in \mathcal{T}^{\prime}$ and on $b_{x_{k}}$ at times $t_{k} \in \mathcal{T} \backslash \mathcal{T}^{\prime}$.

Theorem 2 expresses, in a concise way, that a general process is $K$ classical iff measurements in the computational basis cannot distinguish the action of completely dephasing maps from the action of identity maps. Let us emphasize again that the completely dephasing map not only destroys coherences of the system's reduced state but also quantum correlations between the system and the environment. Therefore, Theorem 2 does not directly link coherence and nonclassicality as Theorem 1 did for the case without memory, where any correlations built up between the system and environment do not affect the subsequent evolution.

Proof.-The proof of Theorem 2 is thus straightforward: If a comb satisfies Eq. (42), then the resulting statistics satisfy Kolmogorov conditions. Conversely, any joint probability distribution on a set of times $\mathcal{T}^{\prime} \subseteq \mathcal{T}$ can either be obtained by direct measurement or by marginalization of the corresponding distribution on $\mathcal{T}$. The former can be computed via the first line of Eq. (42), the latter via the second one. If the statistics of the process appear classical, then both resulting distributions have to coincide, and Eq. (42) must hold.

In the (basis-dependent) Markovian case that we discussed in the previous section, Eq. (42) directly reduces to Eq. (28), i.e., the NCGD property at the level of propagators of populations. Theorem 2 therefore provides the proper generalization of the results of Ref. [34] to the nonMarkovian case. Nonetheless, its consequences for the structural properties of classical combs - and, in particular, the relation of classicality and coherence-remain somewhat opaque in the way Theorem 2 is presently phrased. In order to address these questions, we now introduce a representation of quantum combs that is favorable for the purposes of our work.

\section{B. Choi-Jamiołkowski representation of general quantum processes}

Both the quantum comb describing the $K$ process at hand and the experimental interventions applied at each time are linear maps (the former being a higher-order multilinear map). Any such map can be represented in a variety of ways, but the most natural one for our present purposes makes use of the Choi-Jamiołkowski isomorphism $[87,88]$ between quantum maps and positive semidefinite Hermitian matrices.

A general quantum map-e.g., one that corresponds to a generalized measurement-at time $t_{k}$ is a CP transformation $\mathcal{M}_{x_{k}}: \mathcal{B}\left(\mathcal{H}_{k}^{\mathrm{i}}\right) \rightarrow \mathcal{B}\left(\mathcal{H}_{k}^{\circ}\right)$ that takes bounded linear operators on the (input) Hilbert space $\mathcal{H}_{k}^{i}$ onto bounded linear operators on the (output) Hilbert space $\mathcal{H}_{k}^{\circ}$. Throughout this paper, we consider the input and output spaces of such maps to be isomorphic (and of finite dimension), and the labels $i$ and $\circ$, as well as the time label, are merely introduced for better accounting of the involved spaces. Any such quantum map $\mathcal{M}_{x_{k}}$ can be isomorphically mapped onto a positive semidefinite Hermitian matrix that we call its "Choi state," $M_{x_{k}} \in \mathcal{B}\left(\mathcal{H}_{k}^{\circ} \otimes \mathcal{H}_{k}^{i}\right)$, by letting it act on half of an unnormalized maximally entangled state $\Phi^{+}=\sum_{x_{k}, y_{k}}\left|x_{k} x_{k}\right\rangle\left\langle y_{k} y_{k}\right| \in \mathcal{B}\left(\mathcal{H}_{k}^{i} \otimes \mathcal{H}_{k}^{i}\right)$, i.e.,

$$
M_{x_{k}}:=\left(\mathcal{M}_{x_{k}} \otimes \mathcal{I}\right)\left[\Phi^{+}\right] \in \mathcal{B}\left(\mathcal{H}_{k}^{\circ} \otimes \mathcal{H}_{k}^{i}\right) .
$$

This isomorphism implies, e.g., the following identifications for the identity map, projective measurement map and the completely dephasing map respectively: 


$$
\begin{aligned}
\mathcal{I}_{k} & \Leftrightarrow \Phi_{k}^{+}, \\
\mathcal{P}_{x_{k}} & \Leftrightarrow\left|x_{k}\right\rangle\left\langle x_{k}|\otimes| x_{k}\right\rangle\left\langle x_{k}\right|, \\
\Delta_{k} & \Leftrightarrow \sum_{x_{k}}\left|x_{k} x_{k}\right\rangle\left\langle x_{k} x_{k}\right|:=D_{k} .
\end{aligned}
$$

Here and throughout this article, we typically denote maps with calligraphic uppercase letters (as we have already done above) and their Choi state with the corresponding noncalligraphic variant-with the exception of the identity map [Eq. (44)] and the completely dephasing map [Eq. (46)]. For better orientation, we will continue to denote the respective time at which the maps act by an additional subscript.

Analogously, as a quantum comb $\mathcal{C}_{K}$ is a multilinear map, it can — in a similar way to Eq. (43) — be mapped onto a positive semidefinite Hermitian matrix $C_{K}[35,37,38]$. The action of a quantum comb on a sequence of CP maps $\left\{\mathcal{M}_{x_{K}}, \ldots, \mathcal{M}_{x_{1}}\right\}$ is then equivalently given by [37]

$$
\mathcal{C}_{K}\left[\mathcal{M}_{x_{K}}, \ldots, \mathcal{M}_{x_{1}}\right]=\operatorname{tr}\left[\left(M_{x_{K}}^{\mathrm{T}} \otimes \cdots \otimes M_{x_{1}}^{\mathrm{T}}\right) C_{K}\right],
$$

where $\bullet^{T}$ denotes the transposition with respect to the computational basis. Equation (47) constitutes the Born rule for temporal processes $[89,90]$, where $C_{K}$ plays the role of a quantum state over time and the Choi states $M_{x_{K}}, \ldots, M_{x_{1}}$ play the role that positive operator-valued measure (POVM) elements play in the standard Born rule.

Concretely, given an instrument sequence $\mathcal{J}_{K}, \ldots, \mathcal{J}_{1}$, by combining Eqs. (39) and (47), the joint probability over the sequence of outcomes $x_{K}, \ldots, x_{1}$ is given by

$$
\mathbb{P}_{K}\left(x_{K}, \ldots, x_{1} \mid \mathcal{J}_{K}, \ldots, \mathcal{J}_{1}\right)=\operatorname{tr}\left[\left(M_{x_{K}}^{\mathrm{T}} \otimes \cdots \otimes M_{x_{1}}^{\mathrm{T}}\right) C_{K}\right]
$$

Through this isomorphism, memory effects of the temporal process correspond directly to structural properties of its Choi state [35,40-43]; analogously, the classicality of a process is reflected in the properties of $C_{K}$.

Represented in this way, quantum combs and the channels that they generalize have particularly nice properties. Complete positivity and trace preservation for a quantum channel $\mathcal{M}$ correspond, respectively, to $M \geq 0$ and satisfaction of $\operatorname{tr}_{\circ}[M]=\mathbb{1}_{i}$. Analogously, the Choi state of a quantum comb has to satisfy $C_{K} \geq 0$ as well as a hierarchy of trace conditions that fix the causal ordering of events [37]; i.e., they ensure that later events cannot influence the statistics of earlier ones.

It is important to note that all $K$ processes can be represented through the Choi-Jamiołkowski isomorphism as (unnormalized) quantum states $C_{K}$. In the converse direction, any operator satisfying the aforementioned properties admits an underlying open quantum dynamics description [37,38,51]. Specifically, this means that for every proper comb, there is a (possibly fictitious) environment and a set of system-environment unitaries such that the action of the comb on any sequence of instruments can be written as in Eq. (39). Quantum combs are hence the most general descriptors of open quantum system processes (when the system of interest is probed at fixed times). We call any respective underlying unitary description that includes the environment a "dilation" of the comb. As is the case for quantum channels, any such dilation is nonunique. On the other hand, the comb $\mathcal{C}_{K}$ resulting from some underlying evolution is unique, andjust like the joint probability distribution $\mathbb{P}_{K}$ in the classical case-constitutes the maximal descriptor of the process on the respective set of times.

\section{Structural properties of classical combs}

As a first step to a structural understanding of classical combs, we rephrase Theorem 2 in terms of Choi states:

Theorem $2^{\prime}$. ( $K$-classical quantum combs). A comb $\mathcal{C}_{K}$ on times $\mathcal{T}$, with $|\mathcal{T}|=K$, yields a $K$-classical process iff its Choi state satisfies

$$
\begin{aligned}
& \operatorname{tr}\left[\left(\underset{t_{j} \in \mathcal{T}^{\prime}}{\otimes} \Phi_{j}^{+} \underset{t_{k} \in \mathcal{T} \backslash \mathcal{T}^{\prime}}{\otimes} P_{x_{k}}\right) C_{K}\right] \\
& =\operatorname{tr}\left[\left({\underset{t}{t_{j} \in \mathcal{T}^{\prime}}}_{\boldsymbol{T}^{\prime}} D_{j} \underset{t_{k} \in \mathcal{T} \backslash \mathcal{T}^{\prime}}{\otimes} P_{x_{k}}\right) C_{K}\right] .
\end{aligned}
$$

for all subsets $\mathcal{T}^{\prime} \subseteq \mathcal{T}$ and all possible sequences of outcomes on $\mathcal{T} \backslash \mathcal{T}^{\prime}$.

Using the relations (44)-(46) as well as Eq. (48), it is straightforward to see that this theorem is indeed equivalent to Theorem 2. Importantly, as it is stated in terms of Choi states, Theorem 2' allows one to derive a direct connection between general correlations and the classicality of a $K$ process.

To see how the requirement in Eq. (49) translates to structural constraints on classical combs, first note that any comb that yields the joint probability distribution $\mathbb{P}_{K}\left(x_{K}, \ldots, x_{1}\right)$ when probed in the classical basis can be written as

$$
C_{K}=\tilde{C}_{K}^{\mathrm{Cl}}+\chi,
$$

where the term

$$
\tilde{C}_{K}^{\mathrm{Cl}}=\sum_{x_{K}, \ldots, x_{1}} \mathbb{P}_{K}\left(x_{K}, \ldots, x_{1}\right) P_{x_{K}} \otimes \cdots \otimes P_{x_{1}}
$$

contains the joint probability distribution $\mathbb{P}_{K}$ on its diagonal and $\operatorname{tr}\left[\left(P_{x_{K}} \otimes \cdots \otimes P_{x_{1}}\right) \chi\right]=0$ for all $x_{K}, \ldots, x_{1}[86]$. Intuitively, $\tilde{C}_{K}^{\mathrm{Cl}}$ corresponds to the part of $C_{K}$ that can be probed by measurements in the classical basis alone, while $\chi$ contains all the information about the underlying process that such measurements are blind to. If $\chi=0$, then $C_{K}$ clearly satisfies the conditions of Eq. (42), as $\operatorname{tr}\left[P_{x_{j}} \Phi_{j}^{+}\right]=$ $\operatorname{tr}\left[P_{x_{j}} D_{j}\right]$ for all $x_{j}$ [91]. In other words, for $\chi=0$, the 
corresponding comb is classical, as it is diagonal in the classical product basis. However, the comb need not be diagonal in the classical product basis for Eq. (42) to hold; rather, it suffices if $\chi$ is such that it does not allow one to distinguish between the action of the identity map and the completely dephasing map. We thus arrive at the following lemma:

Lemma 1. Let $C_{K}$ be the comb of a $K$ process on $\mathcal{T}$, with $|\mathcal{T}|=K$, and let $A_{j}:=\Phi_{j}^{+}-D_{j} . C_{K}$ yields a $K$-classical process iff it is of the form

$$
C_{K}=\tilde{C}_{K}^{\mathrm{Cl}}+\chi,
$$

where $\tilde{C}_{K}^{\mathrm{Cl}}$ is obtained from some joint probability distribution $\mathbb{P}_{K}$ via Eq. (51) and $\chi$ satisfies

$$
\operatorname{tr}\left[\left({\underset{t}{t_{j}} \in \mathcal{T}^{\prime}}_{\boldsymbol{T}^{\prime}} A_{j} \underset{t_{k} \in \mathcal{T} \backslash \mathcal{T}^{\prime}}{\otimes} P_{x_{k}}\right) \chi\right]=0
$$

for all subsets $\mathcal{T}^{\prime} \subseteq \mathcal{T}$ and $\mathcal{T}^{\prime}=\varnothing$.

Proof.-It is straightforward to see that a comb of the form of Eq. (52) satisfies Eq. (49), whenever $\chi$ fulfills Eq. (53), and thus yields $K$-classical statistics. Conversely, any comb $C_{K}$ on $K$ times can be written as $C_{K}=\tilde{C}_{K}^{\mathrm{Cl}}+\chi$, where $\tilde{C}_{K}^{\mathrm{Cl}}$ is of the form of Eq. (51) for some $\mathbb{P}_{K}$ and $\operatorname{tr}\left[\left(P_{x_{K}} \otimes \cdots \otimes P_{x_{1}}\right) \chi\right]=0$ [86]. When measuring (in the computational basis) at $K$ times, the resulting joint probability distribution is given by $\mathbb{P}_{K}$. As, by assumption, the process is classical, summation over outcomes obtained at any time $C_{K}$ is defined on must yield the same statistics as letting the comb act on the identity channel at this time. As this has to hold for any collection of times in $\mathcal{T}, \chi$ has to satisfy the additional requirements given by Eq. (53).

Intuitively, Eq. (53) ensures that the action of $\Delta_{j}$ cannot be detected at any point in time by means of measurements in the classical basis. Therefore, Lemma 1 is equivalent to Theorem 2'. However, the former provides an explicit constraint on the structure of such combs that contain coherences that can be present in the process without making the resulting statistics nonclassical.

Indeed, if $\chi=0$, then the corresponding comb $C_{K}$ is diagonal in the classical product basis and, as such, cannot create coherences and destroys any kind of coherences that could be fed into the process (e.g., by performing coherence-creating operations at some time). On the other hand, if $\chi \neq 0$ and the comb contains off-diagonal terms (with respect to the classical basis), then coherences can be created over the course of the process. However, if $\chi$ satisfies Eq. (53), then these coherences-or rather, the invasiveness of the completely dephasing map — cannot be detected at any later time by measurements in the classical basis. This understanding of classical nonMarkovian combs mirrors the intuition that we built in the Markovian setting for the case of NCGD dynamics. Consequently, Lemma 1 fully characterizes the relation between coherences and the nonclassicality of a process

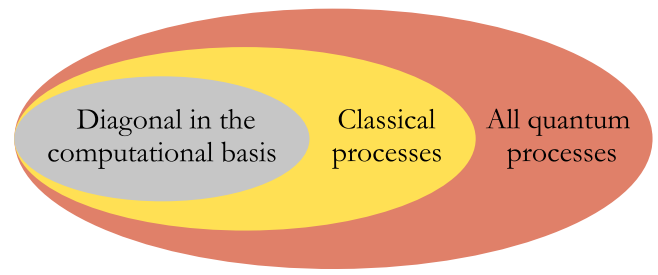

FIG. 7. Nested set of processes. Processes that cannot produce coherence and destroy any coherence that is fed in (i.e., their Choi states are diagonal in the computational basis) form a strict subset of processes that appear classical when sequentially probed in the computational basis. Both of these sets, as well as the set of all quantum processes, are convex.

(see Fig. 7 for a graphical representation of the different sets of processes we consider).

Somewhat unsurprisingly, the above lemma implies that combs leading to classical processes are of measure zero in the set of all combs: While any comb can be written in the form of Eq. (52), Eq. (53) places further linear constraints on the $\chi$ term, which must be satisfied by combs leading to classical processes but not by general combs. The set of combs leading to classical processes is thus confined to a lower-dimensional subset, implying that it is of zero measure (with respect to any reasonable measure in the set of all nonMarkovian combs). This fact falls in line with the intuition built above; for a randomly chosen comb, the action of a completely dephasing map in a given basis will generally be detectable. Furthermore, the vanishing volume of classical combs within the set of all combs mirrors the analogous property in the spatial setting: There, quantum states that display no discord are of measure zero in the set of all bipartite quantum states [92] (the relation between quantum discord and classicality of processes is discussed in detail in Sec. VI).

In the non-Markovian case, the characterization of classical processes comes at a price. In order to decide on the $K$ classicality of a given process, it is no longer sufficient to investigate propagators between pairs of times, but rather, the full part of the comb $C_{K}$ that is relevant for sequential projective measurements must be known, due to the importance of multitime effects. However, this behavior is to be expected, as can already be seen in the case of classical stochastic processes: The full characterization of a nonMarkovian process only happens on the level of the full joint probability distribution $\mathbb{P}_{K}$ and not by way of transition probabilities between adjacent times only. Despite the additional complexity brought in by the presence of memory, as we will see in the following section, measures for classicality that are both experimentally and computationally accessible can be derived based on the characterization of classical processes that we have provided.

\section{Quantifying nonclassicality}

As we have seen above, the set of combs leading to classical processes is of measure zero in the set of all 
combs. Importantly though, this fact does not render our original definition of classicality meaningless, but ratherin conjunction with Lemma 1 -it allows for the derivation of a meaningful measure of nonclassicality that is experimentally accessible and can be formulated by means of a linear program (LP).

More specifically, we can exploit the characterization of classical processes provided by Eqs. (52) and (53) in order to define a measure of nonclassicality with a clear operational meaning. Such a measure not only classifies whether or not a comb is nonclassical but also quantifies the degree to which it is. This quantification is crucial when assessing whether any potential nonclassicality arises from inherently quantum features of the experiment or from experimental errors. In order to clarify its operational interpretation, we formulate our measure in the context of a game with two adversaries, Alice and Bob, and one referee, Rudolph. The task of Alice is to construct a classical stochastic process that is a good model for a comb she receives from Rudolph. The task of Bob is to design a test that distinguishes this model from the original comb. Let $C$ be the given comb in its Choi representation (i.e., a positive operator with some additional causality constraints). The game then proceeds as follows:

(0) Rudolph begins with a given comb $C$ and sends its description to both Alice and Bob.

(A) Alice prepares a classical process $C^{\mathrm{Cl}}$ and sends it to Rudolph.

(R1) Rudolph sends the description of the classical process $C^{\mathrm{Cl}}$ prepared by Alice to Bob.

(B1) Bob prepares a testing sequence $\left\{T_{i}(\vec{x})\right\}_{\vec{x}}$ and sends it to Rudolph.

(R2) Rudolph randomly takes either $C$ or $C^{\mathrm{Cl}}$ and applies the testing sequence chosen by Bob. He yields an outcome $\vec{x}$, which he announces.

(B2) Bob announces whether the comb is $C$ or $C^{\mathrm{Cl}}$.

(R3) Rudolph announces whether Bob is correct or not and hence who wins the game.

Let us recall at this point that our definition of classicality relies exclusively on the statistics obtained by probing the process with projective measurements in fixed, orthonormal bases. Therefore, to only probe what is relevant within our framework, we restrict the testing sequences that Bob is allowed to prepare to only involve such measurements; i.e., the testing sequence must be of the form $T_{i}(\vec{x})=\bigotimes_{t_{j} \in \tau_{i}} \Phi_{j}^{+} \bigotimes_{t_{k} \in \tau_{i}^{c}} P_{x_{k}}$. The figure of merit that we are interested in is the probability for Bob to win if both players play optimally. This operational quantity describes how well said comb can be distinguished from its best classical approximation, given that one only has access to the aforementioned restricted testing strategies that can be used to probe classicality. Making use of the arguments of Lemma 1 to simplify the structure of the classical combs, in Appendix E, we derive this quantity; here, we simply present the main results.
The probability for Bob winning the game is given by

$$
\mathbb{P}_{B}(C)=\frac{1}{2}(1+M(C)),
$$

with $M(C)$ being one-half of the solution of

minimize: $\max _{i} \sum_{\vec{x}}\left|\operatorname{tr}\left[\left(C^{\mathrm{Cl}}-C\right) T_{i}(\vec{x})\right]\right|$

subject to: $C^{\mathrm{Cl}}=\sum_{y_{K}, \ldots, y_{1}} \mathbb{P}_{K}(\vec{y}) P_{y_{K}} \otimes \cdots \otimes P_{y_{1}}$,

$\mathbb{P}_{K}(\vec{y})$ joint prob distribution.

This program can be transformed into the following linear program (and hence can be solved efficiently numerically; the error can be estimated, and one can compute the optimal $C^{\mathrm{Cl}}$ and $\left.T_{i}(\vec{x})[93]\right)$ :

minimize: $a$

$$
\begin{array}{ll}
\text { subject to: } & \sum_{j} b_{i j}-a \leq 0, \\
& \sum_{k} p_{k} \alpha_{i j k}-\beta_{i j}-b_{i j} \leq 0, \\
& -\sum_{k} p_{k} \alpha_{i j k}+\beta_{i j}-b_{i j} \leq 0, \\
& \sum_{k} p_{k}-1=0, \\
& p_{k} \geq 0, a \geq 0, b_{i j} \geq 0,
\end{array}
$$

where we have defined $\alpha_{i j k}:=\operatorname{tr}\left[\left(P_{y_{K}(k)} \otimes \cdots \otimes\right.\right.$ $\left.\left.P_{y_{1}(k)}\right) T_{i}\left(\vec{x}_{j}\right)\right], \beta_{i j}:=\operatorname{tr}\left[C T_{i}\left(\vec{x}_{j}\right)\right]$, and $p_{k}:=\mathbb{P}_{K}(\vec{y}(k))$. For completeness, we also give the dual program, which, by definition, turns a minimization into a maximization. The dual problem is useful to give bounds on the found solution, to solve the problem, and potentially to find different interpretations of the quantity in question. The dual of the program above can be formulated as

$$
\begin{array}{ll}
\text { maximize: } & Z \\
\text { subject to: } & Z \leq \sum_{i j}\left(\alpha_{i j k}-\beta_{i j}\right)\left(2 Y_{i j}-X_{i}\right) \quad \forall k, \\
& \sum_{i} X_{i}=1, \\
& X_{i}, Y_{i j}, X_{i}-Y_{i j} \geq 0, \\
& Z \in \mathbb{R} .
\end{array}
$$

It follows directly from the interpretation as the solution of the game defined above that the quantity $M(C)$ is faithfuli.e., its value is zero if the statistics is classical-and that it measures how difficult it is to simulate the given comb by a classical stochastic process. As such, it provides us with a 
properly motivated quantifier of the degree of nonclassicality of quantum processes, which describes how well the obtained statistics can be simulated by a classical process.

The full evaluation of $M(C)$ would, in principle, require testing over every sequence of projective measurements [to compute the maximization in Eq. (55)] and the comparison with every classical multitime probability distribution [to compute the minimization in Eq. (55)]. Practically, it is then useful to consider bounds to this quantifier of nonclassicality, which can be accessed via a limited number of measurements. In particular, lower bounds can be obtained by using a subset of measurement sequences $T_{i}(\vec{x})$ (in a similar way to how one can use entanglement witnesses to construct bounds on meaningful entanglement measures [94-97]). Finding such a lower bound to be nonzero is already sufficient to conclude that the comb is nonclassical. On the other hand, upper bounds can be attained by restricting our consideration to some classical combs. As a relevant example, for any given comb $C$, one can focus on a single classical comb $\bar{C}^{\mathrm{Cl}}$, realized by applying a dephasing map before and after each measurement. This process yields the statistics resulting from the marginals of the joint statistics one would obtain by measuring at every time. Note that, while this specific choice of a classical comb only provides us with an upper bound on our measure defined above, it is nonetheless faithful. In the simplest case where only two times are involved, $K=2$, one can easily see that by replacing $C^{\mathrm{Cl}}$ with $\bar{C}^{\mathrm{Cl}}$ in Eq. (55), we derive the following upper bound:

$$
M(C) \leq \sum_{x_{2}}\left|\mathbb{P}\left(x_{2}\right)-\sum_{x_{1}} \mathbb{P}\left(x_{2}, x_{1}\right)\right| .
$$

Quantifiers as those on the r.h.s. of Eq. (57) have already been used to investigate coherence properties in transport phenomena [98] and, more recently, to control the departure from any classical random walk via the manipulation of quantum coherence in a time-multiplexed quantum walk experiment [52]. Let us note, at this point, that the experimental data that were used in Ref. [52] to evaluate the right-hand side of Eq. (57) allow one to calculate $M(C)$, too. Hence, $M(C)$ can be evaluated without further acquisition of experimental data, which demonstrates the applicability of our measure to current experiments. In addition, our measure — or lower bounds thereof-can be employed to investigate more complex experiments with $K>2$.

\section{DYNAMICAL PROPERTIES OF $K$-CLASSICAL PROCESSES}

Theorem 2 and Lemma 1 provide a full characterization of processes that yield classical statistics. Together, they allow for the derivation of classically testable quantifiers of nonclassicality. For further clarification, and in order to connect nonclassical processes to the respective underlying evolution, we now discuss some concrete cases of underlying non-Markovian dynamics that lead to classical statistics. Moreover, we connect the classicality of temporal processes to vanishing quantum discord in the joint state of the system and the environment.

\section{A. Discord and classicality}

Recall that in the Markovian case, the classicality of a process can be decided solely in terms of propagators between pairs of times that are defined on the system of interest alone, and it is linked to the ability of those maps to create and detect coherences. In particular, the set of dynamics that does not create coherences on the level of the system is contained in the set of maps that lead to classical statistics [34]. As we have seen above, this fails to hold in the non-Markovian case, where, even if the state of the system is diagonal in the computational basis at all times, i.e., no coherence on the system level is ever generated, the statistics might not satisfy the Kolmogorov conditions.

As soon as memory effects play a non-negligible role, it is both the coherences of the system state and the correlations between the system and its environment that can lead to nonclassical behavior. It is thus desirable to derive a more explicit relation between coherence, correlations, and classicality.

To do so, first recall that while the completely dephasing map leaves the system unchanged if the state of the system is classical at all times, it does not necessarily leave the overall system-environment state-which, at every time $t_{j}$, contains all relevant memory-invariant. Specifically, in this case, we have $\Delta_{j}\left[\rho_{t_{j}}^{s}\right]=\mathcal{I}_{j}\left[\rho_{t_{j}}^{s}\right] \forall t_{j}$ but not necessarily $\Delta_{j} \otimes \mathcal{I}_{j}^{e}\left[\eta_{t_{j}}^{s e}\right]=\mathcal{I}_{j}^{s e}\left[\eta_{t_{j}}^{s e}\right] \forall t_{j}$. While the latter is not necessary for the satisfaction of the Kolmogorov conditions, it is sufficient:

Lemma 2. Let $\left\{p_{t_{i}}^{m}\right\}$ be sets of probabilities that sum to unity, $\left\{\Pi_{j}^{m}\right\}$ orthogonal projectors (not necessarily rank 1) on the system that are diagonal in the computational basis, and $\left\{\xi_{j}^{m}\right\}$ states on the environment. If at all times $t_{j} \in \mathcal{T}$, with $|\mathcal{T}|=K$, the system-environment state is of the form

$$
\eta_{t_{j}}^{s e}=\sum_{m} p_{t_{j}}^{m} \Pi_{j}^{m} \otimes \xi_{j}^{m},
$$

then the underlying process is $K$ classical; i.e., it satisfies the Kolmogorov conditions of Eq. (9).

Note that we assume the computational basis to be the same at every time, so the additional subscript of $\Pi_{j}^{m}$ is somewhat superfluous and merely added to clearly signify the respective time at which the state is defined. In principle, one could define classicality with respect to projective measurements in different bases at each time $t_{j}$, in which case the additional subscript of $\Pi_{j}^{m}$ would denote projectors in different bases, and the above lemma would still hold. Analogously, all other results of this paper can 
straightforwardly be adapted to these more general probing schemes, but for simplicity, we understand classicality with respect to a fixed basis that does not change in time (the only exception being Sec. VII, where we extend the setting to allow for arbitrary measurement schemes in order to examine the nature of genuinely quantum processes.). Naturally, the environment states $\xi_{j}^{m}$ in Eq. (58) can be diagonal in arbitrary bases, as we are only concerned with invasiveness with respect to measurements on the system.

Before we prove Lemma 2, it is insightful to discuss the relation between the concept of classical temporal processes and the classical spatial system-environment correlations it introduces. First, recall that the full system-environment state at each time encapsulates all memory effects. Concretely, in contrast to the state of the system alone, they contain all information that is relevant to predict the future statistics. In particular, for states of the form given in Eq. (58), at each time $t_{j}$, this memory is stored in the probabilities $\left\{p_{t_{j}}^{m}\right\}$ and the environment states $\left\{\xi_{j}^{m}\right\}$. States of said form have vanishing quantum discord [47-50,99]; i.e., they do not display any genuinely quantum correlations between the system and the environment. For a general zero-discord state, the set $\left\{\Pi_{j}^{m}\right\}$ in Eq. (58) could be any set of mutually orthogonal projectors, and the correlations between the system and the environment are considered to be classical since there exists a measurement on the system with perfectly distinguishable outcomes, which, overall, leaves the total state undisturbed $[49,50]$ (see also the proof below).

As we only consider measurements on the system in a fixed basis in our setting, here, vanishing discord at all times does not yet force the resulting statistics to be classical; rather, the discord must vanish in the correct basis, i.e., the one in which the experimenter's measurements act. While discord is often considered as a basis-independent quantity —obtained by a minimization procedure over all possible measurement scenarios [50] here, and throughout the remainder of this article, we always consider its basis-dependent formulation [47$50,61,100]$ and call states of the form in Eq. (58) "discord zero with respect to the classical basis." In other words, whenever we consider a state to be of zero discord, we always implicitly mean that it can be represented as per Eq. (58) with the projectors being diagonal in the classical basis of the measurements. Importantly, this basis dependence mirrors the basis dependence of coherence, which is also always defined with respect to a fixed classical basis.

Proof.-For states of the form in Eq. (58), the completely dephasing map $\Delta$ on the system has the same effect as the "do-nothing" identity channel $\mathcal{I}$, i.e.,

$$
\begin{aligned}
\Delta_{j} & \otimes \mathcal{I}_{j}^{e}\left[\sum_{m} p_{t_{j}}^{m} \Pi_{j}^{m} \otimes \eta_{j}^{m}\right] \\
& =\mathcal{I}_{j}^{s} \otimes \mathcal{I}_{j}^{e}\left[\sum_{m} p_{t_{j}}^{m} \Pi_{j}^{m} \otimes \eta_{j}^{m}\right] .
\end{aligned}
$$

Consequently, if the system-environment state is of this form at all times, the resulting statistics satisfies the Kolmogorov conditions.

It is insightful to reexamine Example 1 in light of Lemma 2. There, we provided an example of a process for which the state of the system never displayed coherence but nonetheless led to nonclassical statistics. Consequently, the system-environment state must have nonzero (basisdependent) discord over the course of the dynamics:

Example 1'. As we discuss in Appendix B, in Example 1 . The system-environment state before the first measurement $\left(t<t_{1}\right)$ is given by

$$
\begin{aligned}
\rho_{s e}(t)= & \frac{1}{4} \sum_{i, j \in\{-,+\}}|i\rangle\langle j| \otimes\left(i \cdot j\left|\psi^{-}(t)\right\rangle\left\langle\psi^{-}(t)\right|\right. \\
& +i(2 \alpha-1)\left|\psi^{-}(t)\right\rangle\left\langle\psi^{+}(t)\right| \\
& +j(2 \alpha-1)\left|\psi^{+}(t)\right\rangle\left\langle\psi^{-}(t)\right| \\
& \left.+\left|\psi^{+}(t)\right\rangle\left\langle\psi^{+}(t)\right|\right),
\end{aligned}
$$

where both

$$
\left|\psi^{+}(t)\right\rangle=\int_{-\infty}^{\infty} d p f(p) e^{i p t}|p\rangle
$$

and

$$
\left|\psi^{-}(t)\right\rangle=\int_{-\infty}^{\infty} d p f(p) e^{-i p t}|p\rangle
$$

are valid quantum states. This state has zero discord with respect to the eigenbasis of $\hat{\sigma}_{x}$ iff

$$
\left|\psi^{+}(t)\right\rangle\left\langle\psi^{+}(t)|-| \psi^{-}(t)\right\rangle\left\langle\psi^{-}(t)\right|=0
$$

and either $\alpha=1 / 2$ or

$$
\left|\psi^{+}(t)\right\rangle\left\langle\psi^{-}(t)|-| \psi^{-}(t)\right\rangle\left\langle\psi^{+}(t)\right|=0 .
$$

In the case of the Lorentzian distribution, it follows from

$$
\left\langle\psi^{-}(t) \mid \psi^{+}(t)\right\rangle=k(t)=e^{-2 \Gamma|t|}
$$

that Eq. (63) cannot be satisfied for $t>0$; i.e., basisdependent discord is created during the evolution (and subsequently destroyed by the measurement at $t_{1}$ ). Since the state of the system itself is not altered by the measurement, but the probabilities to obtain \pm at a later time are (as has been discussed in Ref. [34]), the discord necessarily must be converted into populations by the following portion of evolution. Below, we examine this connection between the creation and detection of basisdependent discord and nonclassicality in a rigorous manner. 
If a state is of zero discord, it displays neither coherences on the level of the system nor nonclassical correlations between the system and the environment, which is, to reiterate, sufficient for the classicality of the resulting process but not necessary. In this sense, Lemma 2 is a direct extension of the analogous statement in the Markovian case; there, the absence of coherence in the system state at all times is also sufficient but not necessary for the process to be classical. Put differently, if all of the individual maps making up a Markovian dynamics are maximally incoherent operations (MIO) $[2,101]$, i.e., they map all incoherent states onto incoherent states, then the resulting dynamics satisfies Kolmogorov conditions. However, MIO operations are a strict subset of NCGD maps [34].

While somewhat intuitive, the above lemma sheds light on the properties that a general non-Markovian dynamics has to satisfy in order to appear classical. For systemenvironment states that are discord zero in the computational basis (with respect to the system), a measurement on the system in the computational basis is noninvasive; i.e., it leaves the full state unchanged (and not just the system state, as would be the case if the system state is incoherent at all times). For comprehensiveness, in Appendix F, we provide a characterization of non-discord-creating processes in terms of their dynamical building blocks.

In general, the absence of discord at all times is not necessary for a process to appear classical. However, what is necessary is that at no time can there be coherences or nonclassical system-environment correlations that can be detected by means of measurements in the computational basis at a later time. This necessity mirrors the requirement for classical processes in the Markovian case, where the individual propagators have to be NCGD; i.e., the propagators must be such that they cannot create coherences whose existence can be picked up at a later time by means of measurements in the classical basis; yet, it is still possible that the individual maps create coherences [34]. NCGD maps are the fundamental building blocks that constitute classical Markovian combs. In what follows, utilizing the connection of classicality and discord discussed above, we provide a characterization of the building blocks that make up classical non-Markovian processes.

\section{B. Non-discord-generating-and-detecting dynamics and classical processes}

In the Markovian case, classicality of a process can be decided on the level of CPTP maps since, in the absence of memory, all higher-order probability distributions can be obtained from the system state $\rho_{t_{1}}$ and the two-time propagators $\left\{\Lambda_{t_{j}, t_{j-1}}\right\}$. It suggests itself to employ this intuition in the non-Markovian case, as every nonMarkovian process corresponds to a Markovian one if enough additional degrees of freedom are taken into account.
In detail, as we discussed, every non-Markovian process can be dilated to a concatenation of a (potentially correlated) system-environment state and unitary total dynamics $[37,38]$, interspersed by the operations of the experimenter on the system alone that are performed at times $\left\{t_{j}\right\}$ (see Fig. 5 for reference). If the experimenter had access to all the degrees of freedom necessary for the dilation, then the underlying process would appear Markovian, and the results of Ref. [34] could be applied on the systemenvironment level for the characterization of a classical process. Here, using the Markovian case as a guideline, we aim for a similar characterization of classical processes when only the system degrees of freedom can be accessed.

To compactify notation and simplify later discussions, we can equivalently consider a general open process as a concatenation of CPTP maps that act on both the system and the environment, interspersed by the operations on the system alone. This way of describing general open system dynamics is simply a notational compression of the general case with global unitaries that allows for an easier connection to the Markovian case but does not lead to a different set of possible combs. In what follows, we denote these CPTP maps by $\Gamma_{t_{j}, t_{j-1}}$ to clearly distinguish them from the memoryless scenario (where the respective maps $\Lambda_{t_{j}, t_{j-1}}$ act only on the system), so Eq. (11) generalizes to

$$
\begin{aligned}
& \mathbb{P}_{n}\left(x_{n}, \ldots, x_{1}\right) \\
& =\operatorname{tr}\left\{\left(\mathcal{P}_{x_{n}} \otimes \mathcal{I}_{n}^{e}\right) \circ \Gamma_{t_{n}, t_{n-1}} \circ \ldots \circ\left(\mathcal{P}_{x_{1}} \otimes \mathcal{I}_{1}^{e}\right)\left[\eta_{t_{1}}^{s e}\right]\right\} .
\end{aligned}
$$

Moreover, for the sake of generality and to ease the comparison with the Markovian case, we allow for the state before the first measurement to be evolved from some other state at an initial reference time $t_{0} \leq t_{1}$, i.e.,

$$
\eta_{t_{1}}^{s e}=\Gamma_{t_{1}, t_{0}} \eta_{t_{0}}^{s e}
$$

of course, if the first measurement occurs at the initial time, then $t_{1}=t_{0}$.

On this dilated level, the dynamics is Markovian - there are no additional external "wires" that can carry memory forward-and all higher-order joint probability distributions could be built up when the individual CPTP maps $\left\{\Gamma_{t_{j}, t_{j-1}}\right\}$ (and the initial system-environment state) are known. Thus, we can define NDGD dynamics:

Definition 3. (NDGD dynamics) A global systemenvironment dynamics with CPTP maps $\left\{\Gamma_{t_{j}, t_{j-1}}\right\}_{j=1}$ is called NDGD if it satisfies

$$
\begin{aligned}
& \Delta_{j+1} \circ \Gamma_{t_{j+1}, t_{j}} \circ \Delta_{j} \circ \Gamma_{t_{j}, t_{j-1}} \circ \Delta_{j-1} \\
& \quad=\Delta_{j+1} \circ \Gamma_{t_{j+1}, t_{j}} \circ \mathcal{I}_{j} \circ \Gamma_{t_{j}, t_{j-1}} \circ \Delta_{j-1}
\end{aligned}
$$

for all $\left\{t_{j-1}, t_{j}, t_{j+1}\right\}$, where the maps $\Gamma_{t_{k}, t_{k-1}}$ act on the system and the environment, while $\Delta_{k}$ acts on the system alone. 


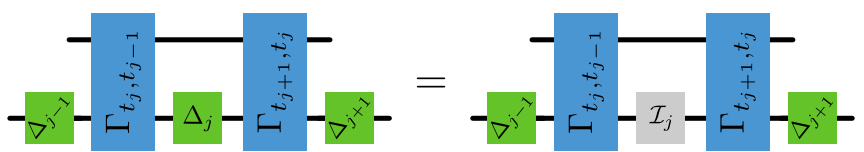

FIG. 8. NDGD system-environment dynamics. From the perspective of a classical observer performing projective measurements in a fixed basis, the identity map at any time $t_{j}$ cannot be distinguished from the completely dephasing map. Any discord (with respect to the classical basis) that is present in the systemenvironment state, and/or created by the system-environment CPTP maps, cannot be detected by such a classical observer.

We provide a graphical representation of this definition in Fig. 8.

Formally, Definition 3 is equivalent to the definition of NCGD dynamics, with the difference that the involved intermediary maps between times are now the systemenvironment maps, instead of the maps $\left\{\Lambda_{t_{j}, t_{j-1}}\right\}$ acting on the system alone in the Markovian case.

Analogously to the case of NCGD, a NDGD dynamics cannot create discord (with respect to the classical basis) that can be detected at the next time (and, as such, at any later time) by means of classical measurements. Or, equivalently, an experimenter who can only perform measurements in the classical basis cannot distinguish between a completely dephasing map and an identity map implemented at any time in $\mathcal{T}$. As such, it provides the natural extension of NCGD to the non-Markovian case. We then have the following theorem:

Theorem 3. (NDGD dynamics and classicality) Consider a general, possibly non-Markovian, process on $\mathcal{T}$, with $|\mathcal{T}|=K$, obtained from a system-environment dynamics as in Eqs. (66) and (67); then, the process is $K$ classical if the initial system-environment state $\eta_{t_{0}}^{\text {se }}$ and the set $\left\{\Gamma_{t_{j}, t_{j-1}}\right\}$ of maps that corresponds to it are zero discord and NDGD, respectively.

The proof of this theorem is provided in Appendix G. It relies on the fact that measurements in the classical basis commute with the completely dephasing map and proceeds along the same lines as the analogous proof for NCGD dynamics in the Markovian setting provided in Ref. [34]. Importantly, though, it is not a necessity for classical statistics that the corresponding maps are NDGD, as we discuss below.

In order to further elucidate the relation of discord and classicality for general quantum stochastic processes, it is insightful to discuss the proximity of Theorem 3 to the corresponding results in Ref. [34] for the Markovian case. Theorem 3 establishes the importance of the role of quantum discord for the classicality of non-Markovian processes. In the memoryless case, it is coherence-or the impossibility of detection thereof-that makes a process classical. Here, this role is played by discord, with the only difference being that instead of describing the process in terms of maps that are solely defined on the system of interest, we are forced to dilate the process to the systemenvironment space, where it is rendered Markovian. Consequently, the classicality of a process cannot be decided based on the master equation or dynamical maps that describe the evolution of the system alone (as has already been pointed out in Ref. [34]). However, given, e.g., a Hamiltonian that generates the corresponding system-environment dynamics, whether or not the resulting process can be simulated classically can be decided by checking the validity of Eq. (68).

It would be desirable for NDGD dynamics to be a sufficient and necessary criterion for the classicality of nonMarkovian processes; however, this is not the case. We provide an example of dynamics that is not NDGD, but nevertheless leads to classical dynamics, in Appendix $\mathrm{H}$. NDGD, as defined in Eq. (68), is a statement about the entire system-environment dynamics and holds for any possible initial state on the environment. However, by means of projective measurements on the system alone, one only has access to the system part, and the systemenvironment dynamics cannot be fully probed. Consequently, the criterion of Eq. (68) will, in general, be too strong for a given experimental scenario. Crucially, though, Theorem 3 allows us to understand the role of the discord generated by the system-environment interaction and subsequently detected via projective measurements on the system in establishing nonclassical statistics.

Nonetheless, even though it is not necessary for the underlying dynamics to be NDGD in order for a nonMarkovian process to display classical statistics, for any $K$ classical process, there always exists a dilation that is NDGD. In other words, there exists a set $\left\{\tilde{\Gamma}_{t_{j}, t_{j-1}}\right\}$ of system-environment CPTP maps that are NDGD and a zero-discord system-environment state $\tilde{\eta}_{t_{0}}^{\text {se }}$ that yields the correct classical family of joint probability distributions when probed in the classical basis. Specifically, we have the following theorem:

Theorem 4. Let $\left\{\mathbb{P}_{n}\left(x_{n}, \ldots, x_{1}\right)\right\}_{n \leq K}$ define a process on $\mathcal{T}$, with $|\mathcal{T}|=K$, coming from an underlying evolution, fixed by the system-environment maps $\left\{\Gamma_{t_{j}, t_{j-1}}\right\}$ and the state $\eta_{t_{0}}^{\text {se }}$, according to Eqs. (66) and (67). The resulting statistics $\left\{\mathbb{P}_{n}\left(x_{n}, \ldots, x_{1}\right)\right\}_{n \leq K}$ is $K$ classical iff there exists a NDGD evolution given by system-environment maps $\left\{\tilde{\Gamma}_{t_{j}, t_{j-1}}\right\}$ defined on times in $\mathcal{T}$ and a zero-discord state $\tilde{\eta}_{t_{0}}^{s e}$ that yield $\mathbb{P}_{n}\left(x_{n}, \ldots, x_{1}\right)$ when probed in the classical basis.

Before we prove this statement, it is important to contrast it with Theorem 1, the analogous result for Markovian processes. There, NCGD propagators of the system dynamics guarantee that the process associated with sequential projective measurements is classical, and classical Markovian processes can be reproduced by a set of NCGD maps (which do not necessarily coincide with the actual dynamical propagators). Analogously, here, the NDGD property of the actual system-environment evolution ensures the classicality 
of the process; while the converse holds for particular dilations, there can be non-NDGD dilations that nonetheless yield classical statistics.

In both cases, the projective measurements in a fixed basis only provide a limited amount of information about the overall evolution underlying the probed statistics. While in the Markovian case the statistics can be traced back to dynamical maps acting on the open system alone, in the more general non-Markovian case, it is the whole system-environment evolution that enters into play. As a consequence, only the former case allows one to establish a one-to-one correspondence between classicality and the properties of the actual evolution by enforcing a proper condition on the dynamics, as discussed at the end of Sec. IVA.

Proof.-As we have already seen in the discussion of Theorem 3, the joint probability distributions obtained from a NDGD dynamics are always classical. We thus only need to prove the opposite direction. Let the underlying system-environment dynamics of the process between times be given by the maps $\left\{\Gamma_{t_{j}, t_{j-1}}\right\}$. As the process is classical, the set of maps $\left\{\tilde{\Gamma}_{t_{j}, t_{j-1}}=\right.$ $\left.\Delta_{j} \circ \Gamma_{t_{j}, t_{j-1}} \circ \Delta_{j-1}\right\}$ together with a state $\tilde{\eta}_{t_{1}}^{s e}=\Delta_{1}\left[\eta_{t_{1}}^{s e}\right]$, where, again, $\Delta_{k}$ only acts on the system degrees of freedom, yields the same joint probability distributions when probed in the classical basis (see Fig. 9 for reference). The process given by this set $\left\{\tilde{\Gamma}_{t_{j}, t_{j-1}}\right\}$ is NDGD by construction, and $\tilde{\eta}_{t_{1}}^{s e}$ has vanishing discord, which means that for every $K$-classical process, there is a NDGD dilation that reproduces it correctly, where we identify the initial time as the time of the first measurement, $t_{0}=t_{1}$.

Theorems 3 and 4 complete our results for the nonMarkovian setting and provide an intuitive connection between nonclassical spatial correlations (i.e., discord) and classical processes.

\section{GENUINELY QUANTUM PROCESSES}

As we have alluded to throughout this article, the classicality of a process depends on the measurement scheme that is employed to probe it; a process that appears classical in one basis - and is thus NDGD with respect to said basis-might display nonclassical correlations when probed differently, thus raising the question of whether nonclassicality is merely a matter of perspective. In principle, for any process, there could exist a probing scheme that yields classical statistics. More concretely, for an experimenter that can perform arbitrary measurements, it might always be possible to "hide" the quantum nature of a process by choosing their respective measurements at the times $\left\{t_{j}\right\}$ such that the resulting statistics are classical.

Naturally, such schemes with (potentially nonprojective) measurements go beyond the discussion of classicality that we have conducted so far. As we will not limit the employed instruments of such schemes to be the same at every time, we

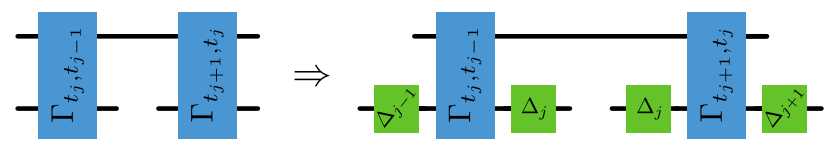

FIG. 9. Transformation to NDGD dilation. Any dilation of an open dynamics can be mapped onto a NDGD one by inserting completely dephasing maps on the level of the system. If the process is classical, then the transformed dilation yields the same statistics as the original one when probed in the classical basis.

call them "unrestricted" in what follows. However, we still assume that the instrument at each time is fixed in advance and is independent of previous measurements - if the choice of instruments depends on previous outcomes, then the employed probing scheme would be temporally correlated and marginalization at a given time would not be well defined.

In this case, our previous results allow us to show that there exist genuinely quantum processes, i.e., processes that display nonclassical statistics with respect to every unrestricted measurement scheme (in the sense described above) that reveals something about the probed process.

To reiterate, up to this point, our discussion of Markovianity has focused on situations where an experimenter measures in the computational basis only, thus employing the same instrument $\mathcal{J}=\left\{\mathcal{P}_{x_{i}}\right\}$ at each time, where all of the (projective) $\mathrm{CP}$ maps $\mathcal{P}_{x_{j}}$ comprising the instrument add up to the completely dephasing map $\Delta_{j}$. More generally, an experimenter could use instruments $\mathcal{J}_{1}=\left\{\mathcal{M}_{x_{1}}\right\}, \mathcal{J}_{2}=\left\{\mathcal{M}_{x_{2}}\right\}, \ldots$, each adding up to the CPTP maps $\mathcal{M}_{1}, \mathcal{M}_{2}, \ldots$, respectively, to sequentially probe the system of interest. Thus, for a process defined on times $\mathcal{T}$, they could collect the joint probability for all subsets $\mathcal{T}^{\prime} \subseteq \mathcal{T}$ and check if Kolmogorov consistency holds. For example, in the simplest case of two times, with $\mathcal{T}=\left\{t_{1}, t_{2}\right\}$ and a given comb $\mathcal{C}_{2}$ on $\mathcal{T}$, an experimenter would consider the process classical, if $\mathbb{P}\left(x_{2} \mid \mathcal{J}_{2}\right)=\sum_{x_{1}} \mathbb{P}\left(x_{2}, x_{1} \mid \mathcal{J}_{2}, \mathcal{J}_{1}\right)$ holds for all $x_{2}$, i.e., if

$$
\mathcal{C}_{2}\left[\mathcal{M}_{x_{2}}, \mathcal{I}_{1}\right]=\mathcal{C}_{2}\left[\mathcal{M}_{x_{2}}, \mathcal{M}_{1}\right] \quad \forall \mathcal{M}_{x_{2}} \in \mathcal{J}_{2} .
$$

Note that, because of causality, the second Kolmogorov condition, i.e., $\mathcal{C}_{2}\left[\mathcal{I}_{2}, \mathcal{M}_{x_{1}}\right]=\mathcal{C}_{2}\left[\mathcal{M}_{2}, \mathcal{M}_{x_{1}}\right]$ for all $\mathcal{M}_{x_{1}} \in$ $\mathcal{J}_{1}$, holds automatically, independent of whether the process is classical or not.

In principle, there could always exist a set of instruments $\left\{\mathcal{J}_{K}, \ldots, \mathcal{J}_{1}\right\}$ for a given process $\mathcal{C}_{K}$ on $\mathcal{T}$, such that the resulting statistics appear classical. Naturally, for this question to make sense, the respective instruments actually have to extract information from the process at hand. In principle, an instrument could consist of a random number generator and a set of CPTP maps that the experimenter implements, depending on the respective output of the random number generator. Considering these outputs as outcomes of the instruments, the experimenter could then collect statistics that are independent of the process at hand 
(they only depend on the statistics of the random number generators) and satisfy Kolmogorov consistency conditions (if the respective random number generators at different times are independent of each other). However, this apparent classicality would not be a statement about the properties of the underlying process, and we thus exclude such pathological instruments. We can do so by demanding that at any time $t_{j}$, none of the elements $\mathcal{M}_{x_{j}}$ of the instrument $\mathcal{J}_{j}$ is proportional to a CPTP map. Under this reasonable assumption, we now show that there are processes that are genuinely quantum; i.e., they violate Kolmogorov conditions for arbitrary choices of instruments.

To this end, in the first step, we argue that genuinely quantum processes can only exist in the non-Markovian setting, while in the memoryless case, there always exists a measurement scheme that yields classical statistics. This conclusion follows from the fact that all features of a Markovian process are governed by the dynamical maps acting on the space of the system alone. Suppose, then, that a Markovian process is deemed to be nonclassical with respect to some basis of measurements, which means that the dynamical maps constituting the process generate and detect coherence with respect to said basis. However, at each point in time throughout the process, the system to be measured is diagonal in some basis (namely, its eigenbasis); thus, in principle, if the experimenter were able to choose an unrestricted measurement scheme that is always diagonal in the same basis as the system, no coherence with respect to this basis will ever be generated and detected, implying that the statistics measured will appear classical. Consequently, in our proposed framework, genuinely quantum processes can only exist in the presence of (quantum) memory.

A similar argument as in the Markovian case holds for the special case of non-Markovian dynamics where the system-environment state at each time is of zero discord in a basis-independent sense, i.e., when there exists a basis with respect to which the joint state at each time has zero discord. Recall that if the system-environment dynamics is NDGD (with respect to a fixed basis), then the statistics observed are classical. Now, if at each time, the systemenvironment state has zero discord, then an experimenter can (in principle) choose the measurement basis at each time to be the one with respect to which the performed measurement is noninvasive. For such a sequence of measurements, the experimenter would not be able to distinguish between having implemented the identity map or the dephasing map (with respect to the chosen basis) at any time since the measurement is noninvasive on the joint system-environment state (due to the lack of discord). Thus, in such a scenario, there always exists some choice of bases in which such a process looks classical. It follows then that no non-Markovian process with zero basis-independent discord between system and environment at every time is genuinely quantum.

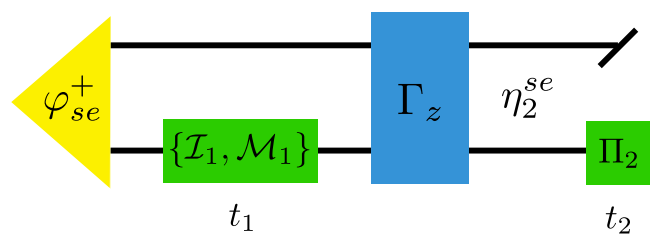

FIG. 10. First two times of a genuinely quantum process. The system and environment begin in a Bell state $\varphi_{s e}^{+}$. Between times $t_{1}$ and $t_{2}$, the map $\Gamma_{z}$ is implemented, which biases the system in the $z$ basis if any CPTP map $\mathcal{M}_{1} \neq \mathcal{I}_{1}$ is performed [see Eq. (70)]. The label $\eta_{2}^{\text {se }}$ refers to the joint system-environment state immediately prior to $t_{2}$ [see Eqs. (71) and (72)]. Classicality implies that the POVM $\Pi_{2}$ must be chosen such that it is unable to detect biases in the $z$ basis. Although this is always possible when only two times are considered, in general, classicality requires satisfaction of a growing number of constraints on the choices of later measurements, which can eventually lead to contradiction, implying the existence of genuinely quantum processes (see Appendix I).

However, the above logic fails in the general setting, which we now show by explicit example. To provide intuition, we first outline the logical implication that is a consequence of the classicality demand for a chosen (twostep) process (depicted in Fig. 10 and described below). While for two times it is always possible to find a measurement scheme such that the statistics appear classical (even in the non-Markovian case), when a non-Markovian process extends over multiple times, finding such a measurement scheme is not possible in general. We show this statement in detail in Appendix I by considering a variant of the process shown in Fig. 10 that is extended over four times, proving the existence of genuinely quantum processes.

The explicit example process we consider begins with an initial system-environment Bell state $\varphi_{s e}^{+}=\frac{1}{2} \sum_{i j}|i i\rangle\langle j j|$. The experimenter can choose to measure the system (in whichever basis, or, more generally, employing any nonpathological instrument they like) at time $t_{1}$. Following this interrogation, the dynamics consists of a systemenvironment CPTP map $\Gamma_{z}: \mathcal{B}\left(\mathcal{H}^{s^{i}} \otimes \mathcal{H}^{e^{i}}\right) \rightarrow \mathcal{B}\left(\mathcal{H}^{s^{\circ}} \otimes \mathcal{H}^{e^{\circ}}\right)$ whose action is to measure its joint inputs in the Bell basis and output the state $\varphi^{+}$if the measurement outcome indeed corresponds to $\varphi^{+}$, or else output a systemenvironment state whose system part is a pure state in the $z$ basis. The action of $\Gamma_{z}$ on a system-environment state $\eta^{\text {se }}$ is thus given by

$$
\begin{aligned}
\Gamma_{z}\left[\eta^{s e}\right]= & \operatorname{tr}\left[\eta^{s e} \varphi_{s e}^{+}\right] \varphi_{s e}^{+} \\
& +\operatorname{tr}\left[\left(\mathbb{1}_{s e}-\varphi_{s e}^{+}\right) \eta^{s e}\right]|0\rangle\left\langle\left. 0\right|_{s} \otimes \tau_{e},\right.
\end{aligned}
$$

where $\tau_{e}$ is some quantum state on the environment. It is straightforward to check that such a map is indeed CPTP. Following this part of the dynamics, the experimenter has access to measure the system at time $t_{2}$. 
For a genuinely quantum process, we demand that the statistics are nonclassical with respect to any possible measurement choices at times $t_{1}$ and $t_{2}$; if this is not the case, then there exists a POVM at $t_{2}$ that cannot distinguish between the experimenter having implemented the identity map $\mathcal{I}_{1}$ or an arbitrary CPTP map $\mathcal{M}_{1}$ at time $t_{1}$, such that the statistics look classical with respect to said measurement scheme. By tracking the joint system-environment state for either choice of operation at $t_{1}$, we first show that for our example, such a POVM always exists, which implies that this is not a genuinely quantum process on two times. However, the POVM that works is constrained by the demand of classicality, as we now detail. Extending the considered process to more times then imposes a number of constraints on the employed measurement devices that must be concurrently satisfied, such that, finally, there is no unrestricted measurement scheme that can yield classical statistics.

Suppose that the experimenter implements $\mathcal{I}_{1}$ at time $t_{1}$; then, the system-environment state at $t_{2}$ is given by

$$
\eta_{2}^{s e}\left(\mathcal{I}_{1}\right):=\Gamma_{z}\left[\left(\mathcal{I}_{1}^{s} \otimes \mathcal{I}^{e}\right)\left[\varphi_{s e}^{+}\right]\right]=\varphi_{s e}^{+},
$$

where the notation $\eta_{2}^{s e}\left(\mathcal{I}_{1}\right)$ refers to the joint state immediately prior to $t_{2}$ given that the experimenter implemented the identity map at $t_{1}$. On the other hand, if the experimenter implements, overall, some CPTP map $\mathcal{M}_{1} \neq \mathcal{I}_{1}$ (corresponding to their instrument $\mathcal{J}_{1}$ at $t_{1}$ ), then the initial Bell pair will be perturbed (as it is only locally invariant under the identity map), and therefore, the system-environment state prior to $t_{2}$ is

$$
\begin{aligned}
\eta_{2}^{s e}\left(\mathcal{M}_{1}\right) & :=\Gamma_{z}\left[\left(\mathcal{M}_{1}^{s} \otimes \mathcal{I}^{e}\right)\left[\varphi_{s e}^{+}\right]\right] \\
& =p \varphi_{s e}^{+}+(1-p)|0\rangle\left\langle\left. 0\right|_{s} \otimes \tau_{e},\right.
\end{aligned}
$$

where $\quad p:=\operatorname{tr}\left[\varphi_{s e}^{+}\left(\mathcal{M}_{1} \otimes \mathcal{I}^{e}\right)\left[\varphi_{s e}^{+}\right]\right]<1$. The statistics observed are gathered by making measurements on only the system, so we are now interested in the reduced system state at $t_{2}$ in either case: From Eq. (71), we have the maximally mixed state $\eta_{2}^{s}\left(\mathcal{I}_{1}\right)=\mathbb{1} / 2$, whereas from Eq. (72), we yield a state that is biased in the $z$ basis, $\eta_{2}^{s}\left(\mathcal{M}_{1}\right)=p 1 / 2+(1-p)|0\rangle\langle 0|$. As previously mentioned, classicality dictates that the POVM implemented at $t_{2}$ must not be able to distinguish between these two states, which leads to the fact that the chosen measurement must be blind to any bias in the $z$ basis. Mathematically, we demand

$$
\mathbb{P}_{2}\left(x_{2} \mid \mathcal{I}_{1}\right) \stackrel{!}{=} \mathbb{P}_{2}\left(x_{2} \mid \mathcal{M}_{1}\right),
$$

which can only be satisfied if the experimenter chooses a POVM $\Pi_{2}=\left\{\Pi_{2}^{\left(x_{2}\right)}\right\}$ such that

$$
\operatorname{tr}\left[\Pi_{2}^{\left(x_{2}\right)} \eta_{2}^{s}\left(\mathcal{I}_{1}\right)\right]=\operatorname{tr}\left[\Pi_{2}^{\left(x_{2}\right)} \eta_{2}^{s}\left(\mathcal{M}_{1}\right)\right] \quad \forall x_{2}
$$

A POVM that satisfies the above equation can be readily constructed: The elements $\left\{\Pi_{2}^{(a)}, \mathbb{1}-\Pi_{2}^{(a)}\right\}$ can always be described by $\Pi_{2}^{(a)}=r_{2}^{(0)} \mathbb{1}+\vec{r}_{2} \cdot \vec{\sigma}$, where $\vec{r}_{2}=\left(r_{2}^{(x)}, r_{2}^{(y)}, r_{2}^{(z)}\right)$ and $\vec{\sigma}=\left(\sigma^{(x)}, \sigma^{(y)}, \sigma^{(z)}\right)$ is the vector of Pauli matrices (note that we have changed notation and use the letter $a$ to label the measurement outcome in order to avoid potential confusion with the $x$-basis direction). Demanding classicality, i.e., Eq. (73), then implies that $r_{2}^{(z)}=0$. In other words, any POVM that is not able to detect biases in the $z$ basis satisfies Eq. (74), and thus, the statistics measured by such a POVM will appear classical. Importantly, here, and in what follows, we can restrict our analysis to the case of POVMs or instruments with only two elements, as any other POVM or instrument (except for the trivial case of single-element ones) can always be coarse grained to a twoelement one. If such a coarse-grained instrument can detect nonclassicality of statistics, then so too can the original one since it necessarily reveals more information about the process upon implementation.

However, although it might be possible to find a basis (or POVM) such that the two-time statistics for a nonMarkovian process look classical, this is not the case in general. Intuitively, demanding that the experimenter cannot distinguish between implementing the identity map and an arbitrary CPTP map at different times leads to a number of constraints (e.g., above, we have the constraint $r_{2}^{(z)}=0$ ) on the later measurement bases. In Appendix I, we consider a process defined across four times that is a logical extension of the two-time process considered here: In each of the first three times, depending on whether or not the system has previously been biased in either the $x, y$, or $z$ basis, the process either performs an identity map (in the affirmative case) or acts to bias the system in one of the bases. In the end, for an arbitrary CPTP map being implemented at each one of the first three times (with the identity map being enacted at the others), the system state at the fourth time is biased in one of the three basis directions, and it is completely unbiased (i.e., maximally mixed) only if three consecutive identity maps are implemented. The only possible POVM at the final time that yields classical statistics must not be able to detect biases in any of the basis vector directions; the only POVM that achieves this is the one with elements proportional to the identity matrix, which corresponds to one of the measurements that we excluded because it reveals nothing about the process. Thus, the process is nonclassical with respect to every possible nonpathological measurement scheme and is therefore genuinely quantum.

A relevant side note seems in order here. Suppose that someone claims that a given process is genuinely quantum. To falsify such a statement, it is enough to probe the process by whatever (nontrivial) devices one chooses; if the statistics one gets is classical, the statement is wrong. The processes that are not genuinely quantum can therefore 
be device-independently verified [102] [103-106]. In turn, this result makes the genuinely quantum processes quite peculiar, as it is impossible to hide their quantumness, and it might be surprising that the set of these processes is nonempty; in fact, we even conjecture that almost all manytime processes are genuinely quantum.

\section{CONCLUSIONS AND OUTLOOK}

\section{A. Conclusions}

In this paper, we have provided an operationally motivated definition of general classical stochastic processes and discussed its structural consequences and relation to quantum coherence in a system's evolution as well as to the generation and activation of nonclassical correlations between the system and the surrounding environment. While we phrased our results predominantly in the language of quantum mechanics, there is - a priori-nothing particularly quantum mechanical about the notion of nonclassicality we introduced. Rather, any process for which the potential invasiveness of performed measurements can be detected by means of said measurements is nonclassical, independent of the underlying theory; as such an invasiveness is experimentally detectable, this is a fully operational notion. The question of whether or not a process is classical can thus be answered on experimentally accessible grounds and is a priori independent of concepts that the experimenter might not be able to check for, like, e.g., coherences in the system of interest.

Nonetheless, our definition allows for the derivation of a direct connection between the classicality of a process and coherences or nonclassical correlations that might be present. While this connection can be formulated in terms of a necessary and sufficient condition for memoryless processes, there are additional subtleties to be considered in the non-Markovian case. In general, it is not sufficient for the state of the system to be diagonal in the classical basis at all times for the resulting multitime statistics to be classical. Rather, it is the interplay of coherences, nonclassical systemenvironment correlations, and the underlying dynamics that is of importance, as we have highlighted through a number of examples presented throughout this paper. Using the comb framework-which can encapsulate this complex interplay - for the description of general quantum processes with memory, we have provided a characterization of quantum processes that yield classical statistics and derived the structural properties of such processes. In principle, analogous structural properties could be derived for processes that display classical statistics when probed by means of different measurements, e.g., nonprojective and/or nonorthogonal ones. However, while still enabling the derivation of structural properties, the clear connection between classicality and quantum discord would be lost as soon as sharp measurements in the computational basis are not the probing mechanism of choice anymore. In this paper, orthogonal projections were chosen as the kind of measurements that come closest to the ideal noninvasiveness displayed by classical measurements. More generally, our results could, in principle, also be extended to postquantum theories. As the definition of classicality we provided is fully operational, the structure of classical processes in such theories could be derived in the same vein as we presented in this paper, with coherence and discord being replaced by the analogous properties of the respective theory.

Unsurprisingly, the set of classical processes turns out to be of measure zero within the set of all quantum processes. The full characterization we have provided equips the set of classical processes with an experimentally accessible measure of nonclassicality that can be formulated as a linear program, thereby providing an operationally clear-cut quantification of the degree of nonclassicality of a given quantum process and a general theoretical framework to define practically useful measures of nonclassicality. As an example, we showed how, within our approach, one can recover and motivate a quantifier of nonclassicality, which is exploited in different contexts [98] and has been used to analyze the properly quantum features of a given experimental setup [52].

Furthermore, we investigated the relation between the nonclassicality of the statistics observed throughout a process and the quantumness of the prevalent spatial system-environment correlations in the underlying dynamics. While the absence of coherence in the state of the system of interest is no longer sufficient in the non-Markovian case to guarantee classicality, the absence of (basis-dependent) discord is. This latter fact is somewhat intuitive, as the absence of discord at all times means that there are neither nonclassical systemenvironment correlations nor coherences in the system that could influence the multitime statistics deduced. Specifically, we have shown that the non-Markovian case, to some extent, mirrors the memoryless one: If the underlying dynamics is NDGD, i.e., any discord that is created at some point in time cannot be detected at a later time, then the process appears classical. While the converse of this statement does not hold, we have further shown that any classical process admits a NDGD dilation.

Finally, we demonstrated that, even if we extend our notion of classicality to the case of unrestricted measurement schemes, there exist processes that display nonclassical statistics independent of how they are probed. This phenomenon can happen only for non-Markovian processes, thus showing that genuine nonclassicality can be seen as a further degree of complexity introduced by the presence of memory effects in the multitime statistics of quantum systems.

As our definition of classicality is tantamount to the assumptions of realism and noninvasiveness that underlie the derivation of Leggett-Garg inequalities, our results furnish experiments that test for the aforementioned properties with a clear interpretation: If the observed statistics 
satisfy a Leggett-Garg inequality, then the underlying process can be assumed to be NDGD. It does not have to be composed of fully classical resources though. On the other hand, violation of a Leggett-Garg inequality implies that quantum discord must have been created (and later detected) over the course of the experiment.

\section{B. Outlook}

While we have provided a comprehensive picture of the interplay between the nonclassical resources that are present in the underlying process and the nonclassicality of the resulting non-Markovian multitime statistics, the mechanisms that lead to the emergence of classical behavior on macroscopic scales remain unclear. Naïvely, the fact that classical processes only constitute a vanishing fraction of the set of all processes renders it puzzling that classical processes can be observed at all. This apparent "puzzle" is reminiscent of the superposition principle, which restricts the set of states that are diagonal in a fixed basis to be of measure zero in the set of all pure states; yet superpositions are generally not observed in the macroscopic domain, where one fixed basis seems to be singled out [107]. While for the latter case, decoherence has been identified as the mechanism that fixes a preferred basis-and as such, leads to the emergence of classicality in the spatial setting $[111,112]$ - an analogous investigation for temporal processes remains outstanding. Our results pave the way towards the analysis of the onset of classicality in general quantum processes when the system and/or environment size increases.

Beyond this foundational perspective, the characterization of the set of classical processes, as well as the measure of nonclassicality we have provided, naturally lends itself to the development of a resource theory of nonclassicality in which processes defined by Eqs. (52) and (53) are free. Additionally, our approach yields a definite theoretical background, which allows one to deal with different quantifiers of the degree of nonclassicality, related to practical situations where different sets of operations are available to investigate the quantumness of physical processes.

On the structural side, we have fully characterized the set of classical processes and have shown that there exist processes that are genuinely quantum. However, the explicit partitioning of the set of quantum processes into classical, nonclassical, and genuinely quantum processes remains opaque and requires further investigation. It suggests itself to assume that the set of genuinely quantum processes is of full measure: As the set of discordant states is of full measure in the set of all states [92], for a randomly chosen process, at any time $t_{j}$, there generally will not exist a measurement that leaves the respective system-environment state invariant, and the subsequent dynamics would have to be highly fine-tuned in order to disguise this invasiveness. More specifically, based on the arguments employed in the explicit construction example of a genuinely quantum process we provided, where four measurement times were necessary to prove the genuine quantumness, we conjecture that almost all processes associated with a $d$-dimensional system are genuinely quantum, if the system is probed $d^{2}$ or more times. A rigorous proof of this statement is the subject of future research. Moreover, since genuinely nonclassical processes lead to nonclassical statistics in a device-independent manner, their quantumness cannot be disguised. It then seems natural to explore if these processes can be used for technological applications.

Finally, the full characterization of general, nonMarkovian quantum processes possessing an equivalent classical description will likely be useful to better understand the different facets of memory effects in the classical and quantum realms. Although the operational framework of quantum combs does not a priori concern any inherent timescales, as the choice of the discrete set of times is arbitrary, from a physical perspective, one expects a connection between some relevant timescales of an underlying system-environment Hamiltonian generating a dynamics and the properties of the corresponding comb that arises upon specification of a set of times. Analogously, the timescalesand number of measurements - over which the nonclassicality of a process can be deduced experimentally will be related to the pertinent timescales of the dynamics. However, determining the properties of an underlying system-environment Hamiltonian that leads to classicality and how the different timescales relate is an interesting, yet multilayered and far from trivial, open problem.

The complexity arises due to the various temporal effects that play a significant role in determining the classicality (or absence thereof) of a given process and the relevant timescales over which it can be detected. For instance, we have already seen that the presence of multitime memory effects is one such property; however, the connection between memory and classicality is a subtle one. One of the key differences between classical and quantum memory effects arises from the generically invasive nature of measurements in quantum mechanics, which leads to an inherent dependence of memory effects on the probing instruments employed [43]. The very notion of relevant memory timescales associated with the evolution of a quantum system therefore crucially depends on whether one wants to infer such timescales via sequential measurements over the course of the evolution or only at some final (possibly varying) time, as is done, e.g., in master equation approaches. In the latter case, the memory of the final statistics on the previous states of the system is dictated by the interplay of different timescales, related to the system of interest, its environment, and their mutual interaction [29]. Such a memory ultimately determines the complexity of the description of the system evolution, as provided, e.g., by memory kernels [113,114], Green functions [115], or path integrals [116]. 
In the case where the temporal correlations of the environment rapidly decay, the process can often be approximated as a Markovian one. When the process is indeed Markovian, i.e., described by a sequence of individual channels between times, as we have shown, it is the NCGD property of the evolution that is necessary and sufficient for classicality; however, this is not easy to relate to the relevant timescales. A property that would be sufficient for classicality, and more straightforwardly related to the inherent timescales of a Hamiltonian generating the evolution, is the forgetfulness of any initial system state.

For instance, suppose one has a Markovian process generated by some Hamiltonian, which has a natural timescale of system forgetfulness, e.g., one that leads to an exponential decay of correlations between any preparations and final measurements. Then, if one probes such a process at sufficiently spaced time instants, one should expect to see classicality: The Markovianity property means that all relevant information can be determined solely on the system level, and forgetfulness ensures that any temporal correlations - in particular, the ability to detect a distinction between a complete dephasing and an identity map-between adjacent times vanish. Strictly speaking, in the standard setting of testing for classicality, where a choice of measurement basis is fixed, one only requires forgetfulness with respect to projective measurements in said basis, rather than complete forgetfulness, for this argument to hold; however, besides being too strict a requirement, connecting such an instrument-specific forgetfulness to the relevant timescales is-like in the NCGD case-a difficult task.

In the presence of memory, the connection between classicality and the relevant timescales of the evolution is more involved yet. Here, we have a subtle interplay between the question concerning the forgetfulness of the system of any initial nonclassicality and how much any nonclassical effects can be transmitted through the environment via the memory mechanism. The fact that forgetfulness of the system alone here is insufficient to imply classicality is related to the crucial point that all multitime effects must be captured in order to properly describe processes with memory. Thus, in the non-Markovian setting, the relevant timescales must typically be determined via sequential measurements over the course of the evolution.

However, different interrogation procedures will lead to the exhibition of different multitime memory effects. For instance, when the system is left unperturbed, the memory can be solely attributed to properties of the underlying Hamiltonian (e.g., those leading to the decay of environmental correlations), whereas when the system is measured, the effect of conditioning the environment state also plays a role. Similarly to the Markovian setting discussed above, the question of classicality of a non-Markovian process does not necessarily concern all such temporal correlations in the process (both those transmitted on the level of the system itself and the genuine memory effects due to the environment) but rather only those that can distinguish between the completely dephasing instrument and the identity map applied to the system. These memory effects are, in turn, a special case of instrument-specific quantum Markov order, which has recently been introduced using the quantum comb formalism $[40,41]$. Connecting such memory effects of the process, and their subsequent impact on the classicality of observed statistics, with the timescales associated with the corresponding Hamiltonian that generates a given process poses a promising avenue for future research.

While we anticipate that the above open questions will generate much theoretical interest, we also expect our results to find immediate application in a broad range of situations where it is relevant to assess whether experimental outcomes are not amenable to a classical description in order to certify some type of quantum advantage or benchmark some genuinely quantum behavior. The former include metrological schemes operating beyond the standard quantum limit [117-120], while the latter can refer to the simulation of many-body quantum systems [121-126]. Also, the role that the emergence of classicality plays in system thermalization and homogenization can be investigated in a systematic and quantitatively tractable manner within our proposed approach.

\section{ACKNOWLEDGMENTS}

Independently from the present work, Philipp Strasberg and María García Díaz derived related results in Ref. [127], where, in particular, the case of non-rank-1 projectors is considered, and a definition of classicality for temporal quantum processes similar to our Theorem 2 is put forward. We thank Konstantin Beyer, Benjamin Desef, María García Díaz, Nana Liu, Kimmo Luoma, Kavan Modi, Felix A. Pollock, Philipp Strasberg, and Walter Strunz for valuable discussions. S. M. is grateful to the Monash Postgraduate Publication Award for financial support. This work was supported by the Swiss National Science Foundation (SNSF, Grant No. P2SKP2_184068); the Austrian Science Fund (FWF): ZK3 (Zukunftkolleg) and Y879N27 (START project); the European Union's Horizon 2020 Research and Innovation Programme under the Marie Skłodowska Curie Grant Agreement No. 801110; the Austrian Federal Ministry of Education, Science and Research (BMBWF); and the ERC Synergy grant BioQ.

\section{APPENDIX A: CONNECTION TO PREVIOUS RESULTS}

In this section, we show that the result derived in the main text for the Markovian case (that is, Theorem 1) implies the preceding one in Ref. [34]. For the ease of the reader, we restate both results here (slightly changing the terminology of the latter to the one used here). 
Theorem 1'. Let $\left\{\mathbb{P}_{n}\left(x_{n}, \ldots, x_{1}\right)\right\}_{n \leq K} \quad$ be a $K$ Markovian process (Definition 2). Then, the process is also $K$ classical (Definition 1 ) if and only if there exists a system state $\rho_{t_{0}}$ (at a time $t_{0} \leq t_{1}$ ), which is diagonal in the computational basis $\{|x\rangle\}_{x \in \mathcal{X}}$, and a set of propagators $\left\{\Lambda_{t_{j}, t_{j-1}}\right\}_{j=1, \ldots, K}$, which are NCGD with respect to $\{|x\rangle\}_{x \in \mathcal{X}}$, such that $\rho_{t_{0}}$ and $\left\{\Lambda_{t_{j}, t_{j-1}}\right\}_{j=1, \ldots, K}$ yield $\left\{\mathbb{P}_{n}\left(x_{n}, \ldots, x_{1}\right)\right\}_{n \leq K}$ via Eq. (16).

Theorem 5. (Theorem 2 of Ref. [34]) Let $\left\{\mathbb{P}_{n}\left(x_{n}, \ldots, x_{1}\right)\right\}_{n \leq K}$ be the process fixed by the QRF, Eq. (16), with respect to a set of propagators forming a CPTP semigroup, i.e., $\Lambda_{t_{l}, t_{j}}=e^{\mathcal{L}\left(t_{l}-t_{j}\right)}$ for any $t_{l} \geq t_{j}$, with $\mathcal{L}$ a Lindblad generator [65,66], and an initial state $\rho_{t_{0}}$. Then, the process $\left\{\mathbb{P}_{n}\left(x_{n}, \ldots, x_{1}\right)\right\}_{n \leq K}$ is $K$ classical (Definition 1) for any $\rho_{t_{0}}$ diagonal in the computational basis if and only if the family of propagators is NCGD in the sense that

$$
\begin{aligned}
& \Delta \circ \Lambda_{s_{3}, s_{2}} \circ \Delta \circ \Lambda_{s_{2}, s_{1}} \circ \Delta \\
& \quad=\Delta \circ \Lambda_{s_{3}, s_{1}} \circ \Delta \quad \forall s_{3} \geq s_{2} \geq s_{1} \geq t_{0} .
\end{aligned}
$$

While the two theorems are clearly related, there are two relevant differences. The new result is more operational in the sense that the statements only depend on the statistics one obtains by making the measurements in the classical basis at the specified times, whereas the statement in Ref. [34] relies on two underlying assumptions on the Markovianity of the quantum dynamics. The first of these assumptions is that the system multitime statistics satisfy the QRF [Eq. (16)], and the second is that the dynamics forms a semigroup. As we see below, the second of these assumptions can be relaxed, but the first is crucial if one wants to have the benefit of the statement in Ref. [34], which not only relates possible models for the statistics [128] but also makes a statement about how the possibility of modeling a process classically implies that the propagators referred to the actual underlying evolution have to satisfy NGCD. To be able to make this connection between the statistics and the underlying quantum evolution, we need to restrict, by assumption, the types of evolutions we are considering. For the Markov case considered here, the natural choice is the QRF [Eq. (16)], as we discussed in the main text that they are closely related.

To prove the connection between the two theorems, it is useful to consider the following corollary to Theorem 1 of the main text:

Corollary 1. Let $\left\{\mathbb{P}_{n}\left(x_{n}, \ldots, x_{1}\right)\right\}_{n \leq K}$ be the process fixed by the QRF, Eq. (16), with respect to a set of divisible propagators and an initial state $\rho_{t_{0}}$.

Let the classical dynamics of this process be invertible, that is, $\mathbb{P}_{1}\left(x_{j}\right) \neq 0$ for an initial diagonal state that is full rank, for any $t_{j}<\infty$. Then, the process $\left\{\mathbb{P}_{n}\left(x_{n}, \ldots, x_{1}\right)\right\}_{n \leq K}$ is $K$ classical (Definition 1) for any $\rho_{t_{0}}$ diagonal in the computational basis if and only if the family of propagators is NCGD, see Eq. (A1).

Proof.-Let $\left\{\mathbb{P}_{n}\left(x_{n}, \ldots, x_{1}\right)\right\}_{n \leq K}$ be a process satisfying the QRF, Eq. (16), with respect to a set of divisible propagators satisfying Eq. (A1). Since the latter implies Eq. (21) and the QRF implies that the process is $K$ Markovian, for any initial diagonal state in the computational basis, $K$ classicality follows from Theorem 1 .

Conversely, let the assumptions hold and the process be $K$ classical, in particular, for an initial diagonal full-rank state. NCGD follows from the equation

$$
\begin{aligned}
& \operatorname{tr}\left\{\mathcal{P}_{x_{3}} \circ \Lambda_{s_{3}, s_{2}} \circ \Delta \circ \Lambda_{s_{2}, s_{1}} \circ \mathcal{P}_{x_{1}} \circ \Lambda_{s_{1}}\left[\rho_{0}\right]\right\} \\
& =\sum_{x_{2}} \operatorname{tr}\left\{\mathcal{P}_{x_{3}} \circ \Lambda_{s_{3}, s_{2}} \circ \mathcal{P}_{x_{2}} \circ \Lambda_{s_{2}, s_{1}} \circ \mathcal{P}_{x_{1}} \circ \Lambda_{s_{1}}\left[\rho_{0}\right]\right\} \\
& =\sum_{x_{2}} \mathbb{P}_{3}\left(x_{3}, x_{2}, x_{1}\right) \\
& =\mathbb{P}_{2}\left(x_{3}, x_{1}\right) \\
& =\operatorname{tr}\left\{\mathcal{P}_{x_{3}} \circ \Lambda_{s_{3}, s_{1}} \circ \mathcal{P}_{x_{1}} \circ \Lambda_{s_{1}}\left[\rho_{0}\right]\right\}
\end{aligned}
$$

(for $s_{3} \geq s_{2} \geq s_{1}$ in $\mathcal{T}$ ) by linearity since, from the assumptions (invertibility of the classical dynamics and taking a diagonal, full-rank initial state), we have that $\mathcal{P}_{x_{1}} \circ \Lambda_{s_{1}}\left[\rho_{0}\right] \neq 0 \forall x_{1}, s_{1}<\infty$ (for $s_{1}, s_{2}, s_{3} \rightarrow \infty, \Lambda_{s_{i}, s_{j}} \rightarrow \mathbb{1}$ and NCGD holds trivially).

The only difference between this corollary and Theorem 2 of Ref. [34] is that here we have the divisibility of the "full" propagators and invertibility of the classical propagators in the assumptions, while there, the dynamics was assumed to be of Lindblad type. However, this latter assumption is strictly stronger, as it implies divisibility and that $\mathcal{P}_{x_{j}} \circ e^{\mathcal{L} t_{j}}[\rho] \neq 0 \forall x_{j}, t_{j}<\infty$ and for any fullrank $\rho$, since (finite-dimensional) semigroup evolutions cannot decrease the rank of a state on a finite time [129].

In total, we have shown, in this section, that Theorem 2 of Ref. [34] can be interpreted as a corollary of Theorem 1 by using the connection between the QRF and Markovianity and further restricting to the case of Lindblad evolution. Moreover, Corollary 1 shows how, by relaxing such a restriction and assuming a proper invertibility condition on the classical dynamics, it is possible to establish a oneto-one correspondence between the classicality of a process satisfying the QRF and the NCGD property, where the latter concerns the propagators of the actual dynamics.

\section{APPENDIX B: ABSENCE OF COHERENCE FOR A MODEL SYSTEM: QUBIT COUPLED TO A CONTINUOUS DEGREE OF FREEDOM}

In this Appendix, we provide the mathematical details missing in the main text for Example 1. We begin with the expression of the global state at time $t_{1}$, immediately before the first measurement: 


$$
\begin{aligned}
\rho_{s e}\left(t_{1}\right)= & \int_{-\infty}^{\infty} d p d p^{\prime} f(p) f^{*}\left(p^{\prime}\right)\left(\rho_{00} e^{i\left(p-p^{\prime}\right) t_{1}}|0 p\rangle\left\langle 0 p^{\prime}\right|\right. \\
& +\rho_{01} e^{i\left(p+p^{\prime}\right) t_{1}}|0 p\rangle\left\langle 1 p^{\prime}\left|+\rho_{10} e^{-i\left(p+p^{\prime}\right) t_{1}}\right| 1 p\right\rangle\left\langle 0 p^{\prime}\right| \\
& \left.+\rho_{11} e^{-i\left(p-p^{\prime}\right) t_{1}}|1 p\rangle\left\langle 1 p^{\prime}\right|\right) .
\end{aligned}
$$

After a measurement at time $t_{1}$ with outcome \pm , the state is subsequently given by

$\rho_{s e}^{( \pm)}\left(t_{1}\right)=| \pm\rangle\left\langle \pm\left|\otimes \int_{-\infty}^{\infty} d p d p^{\prime} f_{1 ; t_{1}}^{( \pm)}\left(p, p^{\prime}\right)\right| p\right\rangle\left\langle p^{\prime}\right|$,

where we emphasize that we have a tensor product state and have introduced the amplitude

$$
\begin{aligned}
f_{1 ; t_{1}}^{( \pm)}\left(p, p^{\prime}\right) \equiv & \frac{1}{C_{t_{1}}^{( \pm)}} f(p) f^{*}\left(p^{\prime}\right)\left(\rho_{00} e^{i\left(p-p^{\prime}\right) t_{1}}\right. \\
& \left. \pm \rho_{01} e^{i\left(p+p^{\prime}\right) t_{1}} \pm \rho_{10} e^{-i\left(p+p^{\prime}\right) t_{1}}+\rho_{11} e^{-i\left(p-p^{\prime}\right) t_{1}}\right)
\end{aligned}
$$

as well as the normalization factor $C_{t_{1}}^{( \pm)}=\int_{-\infty}^{\infty} d p|f(p)|^{2} \times$ $\left(1 \pm 2 \operatorname{Re}\left(\rho_{01} e^{2 i p t_{1}}\right)\right)$. Note that no $\hat{\sigma}_{x}$ coherence is present at this stage.

If we now let the system and environment evolve up to a certain time $\tau>t_{1}$, the global state will be

$$
\begin{aligned}
\rho_{s e}^{( \pm)}(\tau)= & \frac{1}{2} \int_{-\infty}^{\infty} d p d p^{\prime} f_{1 ; t_{1}}^{( \pm)}\left(p, p^{\prime}\right)\left(e^{i\left(p-p^{\prime}\right)\left(\tau-t_{1}\right)}|0 p\rangle\left\langle 0 p^{\prime}\right|\right. \\
& \left. \pm e^{i\left(p+p^{\prime}\right)\left(\tau-t_{1}\right)}|0 p\rangle\left\langle 1 p^{\prime}\right| \pm e^{-i\left(p+p^{\prime}\right)\left(\tau-t_{1}\right.}\right)|1 p\rangle\left\langle 0 p^{\prime}\right| \\
& \left.+e^{-i\left(p-p^{\prime}\right)\left(\tau-t_{1}\right)}|1 p\rangle\left\langle 1 p^{\prime}\right|\right),
\end{aligned}
$$

where the superscript \pm refers to the outcome of the first measurement at time $t_{1}$. The corresponding system state at time $\tau$ is then given by tracing out the environmental degrees of freedom, resulting in

$$
\rho_{s}^{( \pm)}(\tau)=\frac{1}{2}\left(\begin{array}{cc}
1 & \pm k^{( \pm)}\left(\tau, t_{1}\right) \\
\pm k^{( \pm) *}\left(\tau, t_{1}\right) & 1
\end{array}\right),
$$

with

$$
\begin{aligned}
k^{( \pm)}\left(\tau, t_{1}\right)= & \int_{-\infty}^{\infty} d p f_{1 ; t_{1}}^{( \pm)}(p, p) e^{2 i p\left(\tau-t_{1}\right)} \\
= & \frac{1}{C_{t_{1}}^{( \pm)}} \int_{-\infty}^{\infty} d p|f(p)|^{2}\left(1 \pm \rho_{01} e^{2 i p t_{1}}\right. \\
& \left. \pm \rho_{10} e^{-2 i p t_{1}}\right) e^{2 i p\left(\tau-t_{1}\right)} \\
= & \frac{1}{C_{t_{1}}^{( \pm)}}\left(k\left(\tau-t_{1}\right) \pm \rho_{01} k(\tau) \pm \rho_{10} k\left(\tau-2 t_{1}\right)\right) .
\end{aligned}
$$

Once again, we see that if the initial system state is a convex mixture of $|+\rangle$ and $|-\rangle$ and $k(t)$ is real (e.g., a Lorentzian distribution centered at 0 ), then no $\hat{\sigma}_{x}$ coherence is present at any time $\tau$. This fact can be seen because the reduced state can be written as in Eq. (34) for the real $\alpha=\left( \pm k^{( \pm)}\left(\tau, t_{1}\right)+1\right) / 2$. As a side remark, we note that even if the initial state had some coherences with respect to $\hat{\sigma}_{x}$, these would have been destroyed after the first measurement at time $t_{1}$ and, as long as $\rho_{01} \in \mathbb{R}$, would not have been "regenerated" by the subsequent evolution.

Indeed, the argument above can be reiterated for the subsequent measurements; for instance, if we consider the global state after the second measurement at time $t_{2}$, we find

$$
\rho_{s e}^{(s)}\left(t_{2}\right)=| \pm\rangle\left\langle \pm\left|\otimes \int_{-\infty}^{\infty} d p d p^{\prime} f_{2 ; t_{2}, t_{1}}^{(s)}\left(p, p^{\prime}\right)\right| p\right\rangle\left\langle p^{\prime}\right|
$$

with

$$
\begin{aligned}
f_{2 ; t_{2}, t_{1}}^{(s)}\left(p, p^{\prime}\right)= & \frac{1}{C_{t_{2}, t_{1}}^{(s)}} f_{1 ; t_{1}}^{( \pm)}\left(p, p^{\prime}\right)\left(e^{i\left(p-p^{\prime}\right)\left(t_{2}-t_{1}\right)}\right. \\
& +s g(s) e^{i\left(p+p^{\prime}\right)\left(t_{2}-t_{1}\right)}+s g(s) e^{-i\left(p+p^{\prime}\right)\left(t_{2}-t_{1}\right)} \\
& \left.+e^{-i\left(p-p^{\prime}\right)\left(t_{2}-t_{1}\right)}\right)
\end{aligned}
$$

where $s$ denotes the sequence of + and - obtained in the measurements and $s g(s)$ the sign of the corresponding product. The entire procedure can be iterated, by replacing $f_{1 ; t_{1}}^{( \pm)}\left(p, p^{\prime}\right)$ with $f_{2 ; t_{2}, t_{1}}^{(s)}\left(p, p^{\prime}\right)$, so that the state at any subsequent time would remain in the form of Eq. (34), with the off-diagonal elements given by a linear combination with real coefficients of the real function $k(t)$ evaluated at different times. In Appendix C, we show how Example 1 can be described using a comb representation as introduced in Sec. V.

\section{APPENDIX C: COMB REPRESENTATION OF A MODEL SYSTEM: QUBIT COUPLED TO A CONTINUOUS DEGREE OF FREEDOM}

In Appendix B, we showed the absence of coherence in the state of the system at all times for the dynamics of Example 1. To do so, we computed the full systemenvironment dynamics; however, the full knowledge of the system-environment dynamics is not necessary to understand the multitime probabilities of observables of the system alone. Moreover, the state of the environment is often not experimentally accessible in practice, as it is typically highly complex. Therefore, it is convenient to only describe the influence that the environment has on the multitime probabilities. Importantly, this influence, and the 


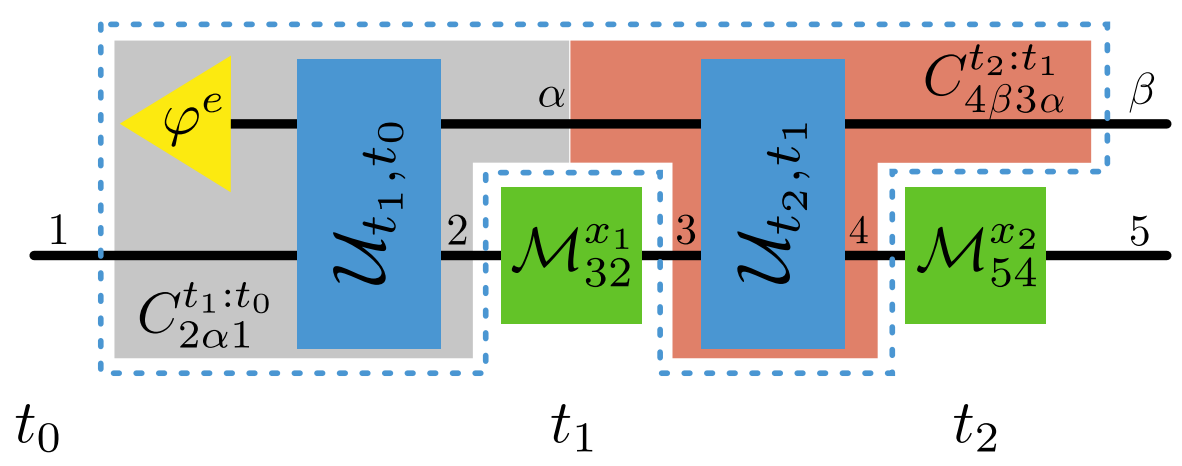

FIG. 11. Labeling of Hilbert spaces used for the comb description of Example 1. The grey box contains the comb $C_{2 \alpha 1}^{t_{1}: t_{0}}$ and the red box the comb $C_{4 \beta 3 \alpha}^{t_{2}: t_{1}}$. The comb $C_{4 \beta 321}^{t_{2}: t_{1}: t_{0}}$ corresponds to everything inside the dashed box and consists of the contraction of the two combs $C_{2 \alpha 1}^{t_{1}: t_{0}}$ and $C_{4 \beta 3 \alpha}^{t_{2}: t_{1}}$.

resulting correct descriptor of the underlying process, can be deduced by probing the system alone.

Such a descriptor can be derived using the concept of quantum combs [37,51], which we briefly reviewed in Sec. V. A quantum comb contains all statistical information that can be inferred about the process it describes (on the set of times upon which it is defined). While here we construct the comb for Example 1 by explicitly solving the systemenvironment dynamics, it is important to note that it could be reconstructed experimentally by means of measurements on the system alone, without any access to or knowledge of the environmental degrees of freedom, through a generalized tomographic scheme [38].

In a slight deviation from the notation of the main text, in this Appendix, for better orientation, here we explicitly write the labels of the Hilbert spaces a comb acts on, and the times it is defined upon, as subscripts and superscripts, respectively.

As described in Example 1, we start with a systemenvironment state $\eta_{s e}\left(t_{0}=0\right)=\rho_{s}\left(t_{0}=0\right) \otimes\left|\varphi^{e}\right\rangle\left\langle\varphi^{e}\right|$, where $\left|\varphi^{e}\right\rangle$ is fixed. As shown in Fig. 11, the initial system state $\rho_{s}\left(t_{0}\right)$ is associated with the Hilbert space with label 1. The channel

$$
\mathcal{C}^{t_{1}: t_{0}}\left(\rho_{s}\right)=\mathcal{U}_{t_{1}, t_{0}} \rho_{s} \otimes\left|\varphi^{e}\right\rangle\left\langle\varphi^{e}\right|
$$

maps the initial system state to the full system-environment state at time $t_{1}$ directly before the intervention. The corresponding channel in the comb description is given by

$$
\begin{aligned}
C_{2 \alpha 1}^{t_{1}: t_{0}} & =\sum_{i, j} U_{t_{1}, t_{0}}\left(| i \rangle \langle j | _ { 2 } \otimes \int _ { - \infty } ^ { \infty } d p \int _ { - \infty } ^ { \infty } d q f ( p ) f ^ { * } ( q ) | p \rangle \langle q | _ { \alpha } ) U _ { t _ { 1 } , t _ { 0 } } ^ { \dagger } \otimes | i \rangle \left\langle\left.j\right|_{1}\right.\right. \\
& =\sum_{i, j} \int_{-\infty}^{\infty} d p \int_{-\infty}^{\infty} d q f(p) f^{*}(q) e^{i\left(\phi_{i} p-\phi_{j} q\right) t_{1}}|i\rangle\left\langle\left. j\right|_{2} \otimes \mid p\right\rangle\left\langle\left. q\right|_{\alpha} \otimes \mid i\right\rangle\left\langle\left. j\right|_{1},\right.
\end{aligned}
$$

where the superscripts denote the intervention times and the subscripts the Hilbert spaces on which the comb is acting. The object $C_{2 \alpha 1}^{t_{1}: t_{0}}$ above is nothing other than the Choi state associated with the channel. The dynamics from time $t_{1}$ to time $t_{2}$ is similarly given by the channel

$$
\mathcal{C}^{t_{2}: t_{1}}\left(\rho_{s e}\right)=\mathcal{U}_{t_{2}, t_{1}} \rho_{s e}
$$

applied to the combined system-environment state directly after the first intervention. Again, this channel admits a Choi state description

$$
C_{4 \beta 3 \alpha}^{t_{2}: t_{1}}=\sum_{i, j} \int_{-\infty}^{\infty} d p \int_{-\infty}^{\infty} d q e^{i\left(\phi_{i} p-\phi_{j} q\right)\left(t_{2}-t_{1}\right)}|i p i p\rangle\left\langle\left. j q j q\right|_{4 \beta 3 \alpha} .\right.
$$

The next step is to eliminate the explicit description of the environment state on Hilbert space $\alpha$. To do this, we contract the Choi states of the two channels described above using the link product $\star$ described in Refs. [37,51]. This process leaves us with the comb describing the dynamics on both times, 


$$
\begin{aligned}
& C_{4 \beta 321}^{t_{2}: t_{1}: t_{0}}=C_{4 \beta 3 \alpha}^{t_{2}: t_{1}} \star C_{2 \alpha 1}^{t_{1}: t_{0}} \\
& =\operatorname{tr}_{\alpha}\left[\left(\mathbb{1}_{4 \beta 3} \otimes C_{2 \alpha 1}^{t_{1}: t_{0} T_{\alpha}}\right)\left(C_{4 \beta 3 \alpha}^{t_{2}: t_{1}} \otimes \mathbb{1}_{21}\right)\right] \\
& =\int_{-\infty}^{\infty} d s\left\langle\left. s\right|_{\alpha} \sum_{i, j} \int_{-\infty}^{\infty} d p \int_{-\infty}^{\infty} d q f(p) f^{*}(q) e^{i\left(\phi_{i} p-\phi_{j} q\right) t_{1}} \mid i q i\right\rangle\left\langle\left. j p j\right|_{2 \alpha 1}\right. \\
& \times \sum_{k, l} \int_{-\infty}^{\infty} d r \int_{-\infty}^{\infty} d t e^{i\left(\phi_{k} r-\phi_{l} t\right)\left(t_{2}-t_{1}\right)}|k r k r\rangle\left\langle\left. l t l t\right|_{4 \beta 3 \alpha} \mid s\right\rangle_{\alpha} \\
& =\sum_{i, j, k, l} \int \ldots \int_{-\infty}^{\infty} d s d p d q d r d t \delta(s-q) \delta(s-t) \delta(p-r) f(p) f^{*}(q) e^{i\left(\phi_{i} p-\phi_{j} q\right) t_{1}}|i i\rangle\left\langle\left. j j\right|_{21}\right. \\
& \times e^{i\left(\phi_{k} r-\phi_{l} t\right)\left(t_{2}-t_{1}\right)}|k r k\rangle\left\langle\left. l t l\right|_{4 \beta 3}\right. \\
& =\sum_{i, j, k, l} \iint_{-\infty}^{\infty} d s d p f(p) f^{*}(s) e^{i\left(\phi_{i} p-\phi_{j} s\right) t_{1}}|i i\rangle\left\langle\left. j j\right|_{21} e^{i\left(\phi_{k} p-\phi_{l} s\right)\left(t_{2}-t_{1}\right)} \mid k p k\right\rangle\left\langle\left. l s\right|_{4 \beta 3}\right. \\
& =\sum_{i, j, k, l} \iint_{-\infty}^{\infty} d s d p f(p) f^{*}(s) e^{i\left(\phi_{i} p-\phi_{j} s\right) t_{1}} e^{i\left(\phi_{k} p-\phi_{l} s\right)\left(t_{2}-t_{1}\right)}|k p k i i\rangle\left\langle\left. l s l j j\right|_{4 \beta 321} .\right.
\end{aligned}
$$

We can also describe the projectors corresponding to the observed measurement outcomes using Choi states, e.g., if we measured in the eigenbasis of $\hat{\sigma}_{x}$ and obtained outcome + , the corresponding Choi state is given by

$$
M^{+}=|+\rangle\left\langle+\left|\otimes \mathbb{1} \sum_{i, j}\right| i i\right\rangle\langle j j||+\rangle\left\langle+\left|\otimes \mathbb{1}=\frac{1}{4} \sum_{i, j, k, l}\right| i j\right\rangle\langle l k| .
$$

Again, using the link product, we can obtain the unnormalized joint system-environment state directly after the second intervention at time $t_{2}$, conditioned on the initial state of the system $\rho_{s}(0)$ and the interventions $M^{x_{1}}, M^{x_{2}}$ (where the superscripts $x_{i}$ refer to the outcomes) as follows:

$$
\begin{aligned}
\rho_{s e}^{\left(x_{2}, x_{1}\right)}\left(t_{2}\right)_{5 \beta} & =C_{4 \beta 321}^{t_{2}: t_{1}: t_{0}} \star \rho_{s}\left(t_{0}\right)_{1} \star M_{32}^{x_{1}} \star M_{54}^{x_{2}} \\
& =\operatorname{tr}_{4321}\left[\rho_{s}\left(t_{0}\right)_{1}^{T} \otimes M_{32}^{x_{1} T_{2}} \otimes M_{54}^{x_{2} T_{4}} C_{4 \beta 321}^{t_{2}: t_{1}: t_{0}}\right] .
\end{aligned}
$$

For instance, if we observed the outcome + twice, the joint state after the second intervention is given by

$$
\begin{aligned}
\rho_{s e}^{(+,+)}\left(t_{2}\right)_{5 \beta}= & \sum_{i, j, k, l, m, n, x, y, a, b, c, d, f, g, h, o}\left\langle\left. f g h o\right|_{4321} \rho_{m n} \mid n\right\rangle\left\langle\left. m\right|_{1} \otimes \frac{1}{4} \mid c x\right\rangle\left\langle\left. d y\right|_{32} \otimes \frac{1}{2} \mid+\right\rangle\left\langle+\left.\right|_{5} \otimes \mid a\right\rangle\left\langle\left. b\right|_{\beta}\right. \\
& \times \iint_{-\infty}^{\infty} d s d p f(p) f^{*}(s) e^{i\left(\phi_{i} p-\phi_{j} s\right) t_{1}}|i i\rangle\left\langle\left. j j\right|_{21} e^{i\left(\phi_{k} p-\phi_{l} s\right)\left(t_{2}-t_{1}\right)} \mid k p k\right\rangle\left\langle\left. l s\right|_{4 \beta 3} \mid f g h o\right\rangle_{4321} \\
= & \frac{1}{8}|+\rangle\left\langle+\left.\right|_{5} \otimes \sum_{i, j, k, l} \rho_{i j} \iint_{-\infty}^{\infty} d s d p f(p) f^{*}(s) e^{i\left(\phi_{i} p-\phi_{j} s\right) t_{1}} e^{i\left(\phi_{k} p-\phi_{l} s\right)\left(t_{2}-t_{1}\right)} \mid p\right\rangle\left\langle\left. s\right|_{\beta}\right. \\
= & \frac{1}{8}|+\rangle\left\langle+\left.\right|_{5} \otimes \iint_{-\infty}^{\infty} d p d s \tilde{f}_{2 ; t_{2}, t_{1}}^{(+,+)}(p, s) \mid p\right\rangle\left\langle\left. s\right|_{\beta},\right.
\end{aligned}
$$

where we have introduced

$$
\begin{aligned}
\tilde{f}_{2 ; t_{2}, t_{1}}^{(+,+)}(p, s)= & \sum_{i, j, k, l} \rho_{i j} f(p) f^{*}(s) e^{i\left(\phi_{i} p-\phi_{j} s\right) t_{1}} e^{i\left(\phi_{k} p-\phi_{l} s\right)\left(t_{2}-t_{1}\right)} \\
= & f(p) f^{*}(s)\left(\rho_{00} e^{i(p-s) t_{1}}+\rho_{01} e^{i(p+s) t_{1}}+\rho_{10} e^{-i(p+s) t_{1}}+\rho_{11} e^{-i(p-s) t_{1}}\right) \\
& \times\left(e^{i(p-s)\left(t_{2}-t_{1}\right)}+e^{i(p+s)\left(t_{2}-t_{1}\right)}+e^{-i(p+s)\left(t_{2}-t_{1}\right)}+e^{-i(p-s)\left(t_{2}-t_{1}\right)}\right) \\
= & f_{2 ; t_{2}, t_{1}}^{(+,+)}(p, s)
\end{aligned}
$$

and checked the consistency with the direct description in Appendix B. 


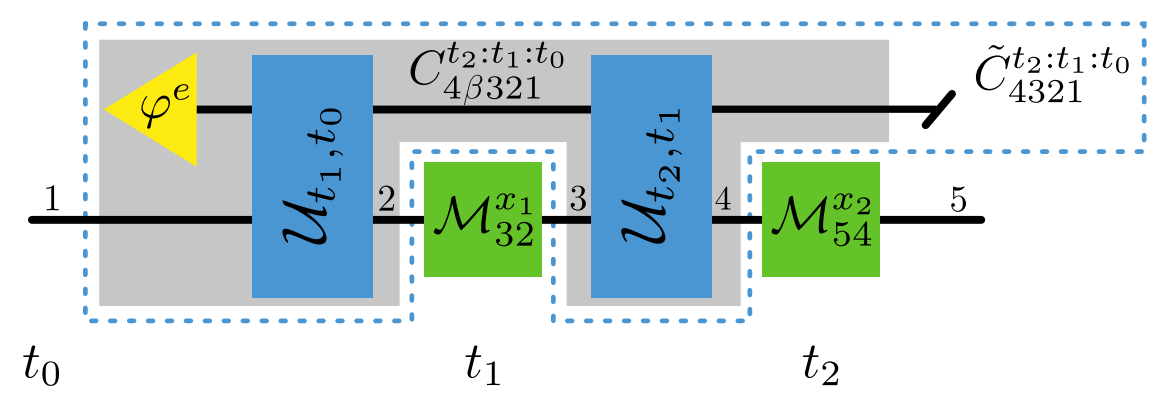

FIG. 12. Dilation for Example 1. Pictorial representation of the quantum combs describing Example 1 with two interventions.

Since we are mainly interested in the question of whether the obtained measurement statistics can be explained classically, we restrict our attention to the unnormalized state of the system alone because the probability of obtaining a specific sequence of measurement outcomes is encoded in the trace of the corresponding system state. Therefore, we eliminate the description of the environment by tracing over the Hilbert space $\beta$, which we can do directly at the level of the comb itself,

$$
\begin{aligned}
\tilde{C}_{4321}^{t_{2}: t_{1}: t_{0}}= & \operatorname{tr}_{\beta}\left[C_{4 \beta 321}^{t_{2}: t_{1}: t_{0}}\right] \\
= & \sum_{i, j, k, l} \int_{-\infty}^{\infty} d q|f(q)|^{2} e^{i\left(\phi_{i}-\phi_{j}\right) q t_{1}} \\
& \times e^{i\left(\phi_{k}-\phi_{l}\right) q\left(t_{2}-t_{1}\right)}|k k i i\rangle\left\langle\left. l l j j\right|_{4321} .\right.
\end{aligned}
$$

Following the same procedure as above, we then obtain the system state after the second intervention,

$$
\rho_{s}^{\left(x_{2}, x_{1}\right)}\left(t_{2}\right)_{5}=\tilde{C}_{4 \beta 321}^{t_{2}: t_{1}: t_{0}} \star \rho_{s}\left(t_{0}\right)_{1} \star M_{32}^{x_{1}} \star M_{54}^{x_{2}} .
$$

Similarly, the probability to obtain, e.g., twice the measurement result + is given by

$$
\mathbb{P}_{2}\left(+, t_{2} ;+, t_{1}\right)=\operatorname{tr}\left[\rho_{s}^{(+,+)}\left(t_{2}\right)_{5}\right]
$$

If we introduce $\tau_{n}:=t_{n}-t_{n-1}$, by way of induction, we find that

$$
\begin{aligned}
C^{t_{n}}: t_{0} & =\sum_{i_{2 n} \ldots i_{1}, j_{2 n} \ldots j_{1}} \iint_{-\infty}^{\infty} d p d q f(p) f^{*}(q)|p\rangle\left\langle q\left|\bigotimes_{a=1}^{2 n} e^{i\left(\phi_{i_{a}} p-\phi_{j_{a}} q\right) \tau_{a}}\right| i_{a} i_{a}\right\rangle\left\langle\left. j_{a} j_{a}\right|_{2 a, 2 a-1},\right. \\
\tilde{C}^{t_{n}:: t_{0}} & =\sum_{i_{2 n} \ldots i_{1}, j_{2 n} \ldots j_{1}} \int_{-\infty}^{\infty} d p|f(p)|^{2} \bigotimes_{a=1}^{2 n} e^{i\left(\phi_{i_{a}}-\phi_{j_{a}}\right) p \tau_{a}}\left|i_{a} i_{a}\right\rangle\left\langle\left. j_{a} j_{a}\right|_{2 a, 2 a-1},\right.
\end{aligned}
$$

where we suppressed the subscripts of the combs. As above, $C^{t_{n}:: t_{0}}$ denotes the comb including the outgoing environment and $\tilde{C}^{t_{n}:: t_{0}}$ the comb describing the system alone; see Fig. 12 for a pictorial representation. Therefore, the joint probability distribution for sequences of measurement outcomes is given by

$$
\mathbb{P}_{n}\left(x_{n}, t_{n} ; \ldots ; x_{1}, t_{1}\right)=\operatorname{tr}\left[\rho_{s}(0)_{1}^{T} \bigotimes_{a=1}^{n}\left(M_{2 a+1,2 a}^{x_{a}}\right)^{T} \tilde{C}^{t_{n}:: t_{0}}\right]
$$

\section{APPENDIX D: ALTERNATIVE EXAMPLE FOR NONCLASSICAL DYNAMICS THAT DO NOT CREATE COHERENCES}

Here, we provide an alternative example of a process where the state of the system is diagonal in the computational basis at all times but does not yield classical statistics.
To this end, consider the following circuit (see Fig. 13): Let the initial system-environment state at time $t_{0}$ be a maximally entangled two-qubit state $\varphi^{+}$that undergoes trivial evolution between $t_{0}$ and $t_{1}$. At $t_{1}$, the system alone is thus in a maximally mixed state $\rho_{t_{1}}=\mathbb{1} / 2$. Between $t_{1}$ and $t_{2}$, the system and the environment undergo a CPTP map $\mathcal{E}_{t_{2}, t_{1}}$ (which could, in principle, be dilated to a unitary map [130], but for conciseness, we restrict ourselves to the relevant part of it), which yields output $|0\rangle$ on the system, if the system and environment are in the state $\varphi^{+}$, and $|1\rangle$ otherwise, i.e., when the system-environment state is orthogonal to $\varphi^{+}$. Consequently, its action can be written as

$$
\mathcal{E}_{t_{2}, t_{1}}[\eta]=\operatorname{tr}\left(\varphi^{+} \eta\right)|0\rangle\left\langle 0\left|+\operatorname{tr}\left[\left(\mathbb{1}-\varphi^{+}\right) \eta\right]\right| 1\right\rangle\langle 1| .
$$

It is easy to check that $\mathcal{E}_{t_{2}, t_{1}}$ is indeed CPTP, and the state of the system at $t_{2}$ is a convex mixture of $|0\rangle\langle 0|$ and $|1\rangle\langle 1|$ for 


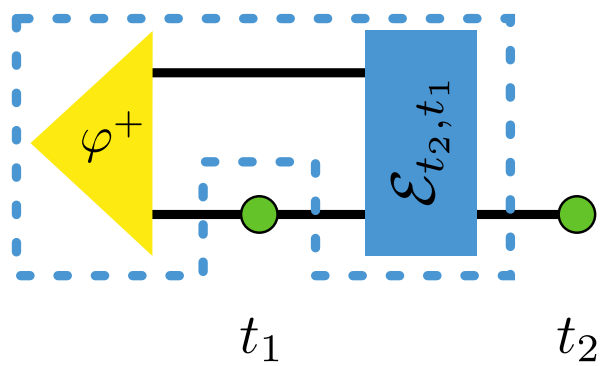

FIG. 13. Nonclassical process that does not display coherences. The state of the system is classical; i.e., it does not contain coherences with respect to the classical basis, at any step of the process. However, the corresponding statistics do not satisfy the Kolmogorov conditions. Potential measurements are depicted as green circles. The blue dotted line signifies the comb of the process (see Sec. V).

all possible experimental interventions at $t_{1}$; there are thus no coherences in the state of the system at any of the times $\left\{t_{1}, t_{2}\right\}$. However, this process does not satisfy the Kolmogorov consistency.

To see this, consider the probabilities for a measurement in the computational basis at $t_{2}$, with no operation performed at $t_{1}$. In this case, the system-environment state before the action of $\mathcal{E}_{t_{2}, t_{1}}$ is equal to $\varphi^{+}$, which means that we have $\rho_{t_{2}}=|0\rangle\langle 0|$. Consequently, a measurement in the computational basis at $t_{2}$ yields the probabilities

$$
\mathbb{P}_{1}\left(0, t_{2}\right)=1 \quad \text { and } \quad \mathbb{P}_{1}\left(1, t_{2}\right)=0
$$

On the other hand, performing a measurement at $t_{1}$ and discarding the outcomes amounts to performing the completely dephasing map $\Delta_{1}$. Immediately after this map, i.e., right before $\mathcal{E}_{t_{2}, t_{1}}$, the system-environment state is of the form

$$
\eta_{t_{1}}^{s e}=\frac{1}{2} \sum_{x_{1}}\left|x_{1}\right\rangle\left\langle x_{1}|\otimes| x_{1}\right\rangle\left\langle x_{1}\right|=\frac{1}{2}\left(\varphi^{+}+\varphi^{-}\right)
$$

where $\varphi^{-}=\left(\sigma_{z} \otimes \mathbb{1}\right) \varphi^{+}\left(\sigma_{z} \otimes \mathbb{1}\right) \quad$ is a $\quad$ Bell state. Consequently, in this case, the final system state $\rho_{t_{2}}$ is of the form $\rho_{t_{2}}=\frac{1}{2}(|0\rangle\langle 0|+| 1\rangle\langle 1|)$. Finally, the obtained probabilities for a measurement in the computational basis at $t_{2}$ are

$$
\begin{aligned}
\mathbb{P}_{1}^{\Delta_{1}}\left(0, t_{2}\right) & =\sum_{x_{1}} \mathbb{P}_{2}\left(x_{1}, t_{1} ; 0, t_{2}\right)=\frac{1}{2} \\
\text { and } \quad \mathbb{P}_{1}^{\Delta_{1}}\left(1, t_{2}\right) & =\sum_{x_{1}} \mathbb{P}_{2}\left(x_{1}, t_{1} ; 1, t_{2}\right)=\frac{1}{2},
\end{aligned}
$$

which do not coincide with Eq. (D2). Even though the state of the system is incoherent at all considered times, i.e., the state itself appears to be classical, the multitime statistics do not satisfy the Kolmogorov condition.

\section{APPENDIX E: MEASURE OF NONCLASSICALITY}

In this Appendix, we derive the optimal solution of the game, which defines our measure of nonclassicality $M(C)$, and show that it can be formulated as a linear program. We also derive the dual of this problem for completeness.

In our game, Bob can choose the points in time at which he wants Rudolph to perform projective measurements and those for which Rudolph should not interfere with the natural evolution of the system. Bob's choices define a sequence of measurements $T_{i}(\vec{x})=\bigotimes_{t_{j} \in \tau_{i}} \Phi_{j}^{+}$ $\otimes_{t_{k} \in \tau_{i}^{c}} P_{x_{k}}$. Given the choice of any sequence of this form and labeling the obtained outcome sequence of the experiment by $\vec{x}$, the best strategy for Bob is to announce that the comb that was tested is $C$ if the probability for measuring outcome $\vec{x}$ with said sequence $T_{i}(\vec{x})$ is higher for $C$ than for $C^{\mathrm{Cl}}$ [i.e., if $\left.\operatorname{tr}\left[\left(C^{\mathrm{Cl}}-C\right) T_{i}(\vec{x})\right]<0\right]$ and announcing $C^{\mathrm{Cl}}$ otherwise. The probability that he is correct when announcing $C$, given that the outcome obtained was $\vec{x}$, is given by

$$
\mathbb{P}(C \mid \vec{x})=\frac{\mathbb{P}(C, \vec{x})}{\mathbb{P}(\vec{x})}=\frac{\mathbb{P}(\vec{x} \mid C)}{\mathbb{P}(\vec{x})} \mathbb{P}(C),
$$

where the prior probability is $\mathbb{P}(C)=1 / 2$. Denoting by $S^{\mathrm{Cl}}$ the set of all $\vec{x}$ such that $\operatorname{tr}\left[\left(C^{\mathrm{Cl}}-C\right) T_{i}(\vec{x})\right]>0$ and $S_{c}^{\mathrm{Cl}}$ its complement, the probability that Bob wins the game is given by

$$
\begin{aligned}
& \sum_{\vec{x} \in S_{c}^{\mathrm{Cl}}} \mathbb{P}(C \mid \vec{x}) \mathbb{P}(\vec{x})+\sum_{\vec{x} \in S^{\mathrm{Cl}}} \mathbb{P}\left(C^{\mathrm{Cl}} \mid \vec{x}\right) \mathbb{P}(\vec{x}) \\
& =\frac{1}{2}\left(\sum_{\vec{x} \in S_{c}^{\mathrm{Cl}}} \mathbb{P}(\vec{x} \mid C)+\sum_{\vec{x} \in S^{\mathrm{Cl}}} \mathbb{P}\left(\vec{x} \mid C^{\mathrm{Cl}}\right)\right) \\
& =\frac{1}{2}\left(\sum_{\vec{x} \in S_{c}^{\mathrm{Cl}}} \operatorname{tr}\left[C T_{i}(\vec{x})\right]+\sum_{\vec{x} \in S^{\mathrm{Cl}}} \operatorname{tr}\left[C^{\mathrm{Cl}} T_{i}(\vec{x})\right]\right) \\
& =\frac{1}{2}\left(1+\sum_{\vec{x} \in S^{\mathrm{Cl}}}\left(-\operatorname{tr}\left[C T_{i}(\vec{x})\right]+\operatorname{tr}\left[C^{\mathrm{Cl}} T_{i}(\vec{x})\right]\right)\right) .
\end{aligned}
$$

Assuming that both Alice and Bob play ideally, using Lemma 1 , the probability $\mathbb{P}_{B}(C)$ that Bob wins is given by

$$
\mathbb{P}_{B}(C)=\frac{1}{2}(1+M(C)),
$$

where $M(C)$ is the solution of 


$$
\text { minimize: } \max _{i} \sum_{\vec{x} \in S^{\mathrm{Cl}}} \operatorname{tr}\left[\left(C^{\mathrm{Cl}}-C\right) T_{i}(\vec{x})\right]
$$

subject to: $S^{\mathrm{Cl}}=\left\{\vec{x} \mid \operatorname{tr}\left[\left(C^{\mathrm{Cl}}-C\right) T_{i}(\vec{x})\right] \geq 0\right\}$,

$$
\begin{aligned}
& C^{\mathrm{Cl}}=\sum_{y_{K}, \ldots, y_{1}} \mathbb{P}_{K}(\vec{y}) P_{y_{K}} \otimes \cdots \otimes P_{y_{1}}+\chi, \\
& \operatorname{tr}\left[\chi \cdot\left(\otimes_{t_{j} \in \tau_{i}} A_{j} \otimes_{t_{k} \in \tau_{i}^{c}} P_{z_{k}}\right)\right]=0, \\
& C^{\mathrm{Cl}} \geq 0, \\
& \operatorname{tr}_{K^{\mathrm{i}}}\left[C^{\mathrm{Cl}}\right]=\mathbb{1}_{(K-1)^{\circ}} \otimes \Theta_{K-1}, \\
& \vdots \\
& \operatorname{tr}_{2^{\mathrm{i}}}\left[\Theta_{2}\right]=\mathbb{1}_{1^{\circ}} \otimes \rho_{1^{\mathrm{i}}}, \\
& \mathbb{P}_{K}(\vec{y}) \text { joint prob distribution, }
\end{aligned}
$$

where we defined $A_{j}:=\Phi_{j}^{+}-D_{j}$ and $\rho_{1^{\mathrm{i}}}$ is a valid quantum state. The hierarchy of partial trace conditions on the comb written above ensures that the overall action of any instrument at a later time cannot influence previous statistics [36,37].

Starting from the above program, we see that $\chi$ does not contribute to the trace, as $\operatorname{tr}\left[\chi T_{i}(\vec{x})\right]$ is, by definition, a marginal of a zero distribution (due to the third constraint above); see also the proof of Lemma 1 . This result leaves us with contributions only from the diagonal parts of the operator $C^{\mathrm{Cl}}$, where the nonzero entries are those that correspond to $\mathbb{P}_{K}(\vec{y}) P_{y_{K}} \otimes \cdots \otimes P_{y_{1}}$, which must satisfy $\operatorname{tr}\left[C^{\mathrm{Cl}}\right]=1$ and $C^{\mathrm{Cl}} \geq 0$ due to the requirement that $\mathbb{P}_{K}(\vec{y})$ is a valid probability distribution. Note that for any such operator, there exists a $\chi$ such that the total operator satisfies the additional requirements in the above program since one simply must add terms of the form $\sum_{y_{K}, \ldots, y_{1}} \mathbb{P}_{K}(\vec{y}) P_{y_{K}, z_{K}} \otimes$ $\cdots \otimes P_{y_{1}, z_{1}}$, where the $P_{y_{j}, z_{j}}$ are projectors up to a permutation on the input basis (i.e., $P_{y_{j}, z_{j}}=\left|y_{j}\right\rangle\left\langle\left. y_{j}\right|_{\circ} \otimes \mid z_{j}\right\rangle\left\langle\left. z_{j}\right|_{i}\right.$ ). We are then left with

$$
\begin{aligned}
& \text { minimize: } \max _{i} \sum_{\vec{x} \in S^{\mathrm{Cl}}} \operatorname{tr}\left[\left(C^{\mathrm{Cl}}-C\right) T_{i}(\vec{x})\right] \\
& \text { subject to: } S^{\mathrm{Cl}}=\left\{\vec{x} \mid \operatorname{tr}\left[\left(C^{\mathrm{Cl}}-C\right) T_{i}(\vec{x})\right] \geq 0\right\}, \\
& C^{\mathrm{Cl}}=\sum_{y_{K}, \ldots, y_{1}} \mathbb{P}_{K}(\vec{y}) P_{y_{K}} \otimes \cdots \otimes P_{y_{1}}, \\
& \mathbb{P}_{K}(\vec{y}) \text { joint prob distribution. }
\end{aligned}
$$

Since both $C$ and $C^{\mathrm{Cl}}$ represent (up to a noncontributing $\chi$ ) deterministic quantum combs, we have

$$
\sum_{\vec{x}} \operatorname{tr}\left[\left(C^{\mathrm{Cl}}-C\right) T_{i}(\vec{x})\right]=0
$$

and thus

$$
\sum_{\vec{x}}\left|\operatorname{tr}\left[\left(C^{\mathrm{Cl}}-C\right) T_{i}(\vec{x})\right]\right|=2 \sum_{\vec{x} \in S^{\mathrm{Cl}}} \operatorname{tr}\left[\left(C^{\mathrm{Cl}}-C\right) T_{i}(\vec{x})\right]
$$

This result allows us to express $M(C)$ as half of the solution of

$$
\begin{aligned}
& \text { minimize: } \max _{i} \sum_{\vec{x}}\left|\operatorname{tr}\left[\left(C^{\mathrm{Cl}}-C\right) T_{i}(\vec{x})\right]\right| \\
& \text { subject to: } C^{\mathrm{Cl}}=\sum_{y_{K}, \ldots, y_{1}} \mathbb{P}_{K}(\vec{y}) P_{y_{K}} \otimes \cdots \otimes P_{y_{1}}, \\
& \mathbb{P}_{K}(\vec{y}) \text { joint prob distribution. }
\end{aligned}
$$

In order to transform this program into a $\mathrm{LP}$, for every testing sequence $\left\{T_{i}(\vec{x})\right\}_{\vec{x}}$, we define an arbitrary order of the outcomes $\vec{x}$; i.e, we label them as $\vec{x}_{j}$. Then,

$$
\max _{i} \sum_{\vec{x}}\left|\operatorname{tr}\left[\left(C^{\mathrm{Cl}}-C\right) T_{i}(\vec{x})\right]\right|
$$

is the solution of

minimize: $a$

subject to: $a \geq \sum_{j}\left|\operatorname{tr}\left[\left(C^{\mathrm{Cl}}-C\right) T_{i}\left(\vec{x}_{j}\right)\right]\right|$,

which is equivalent to

minimize: $a$

subject to: $a \geq s_{i}$,

$$
\begin{aligned}
s_{i} & =\sum_{j} b_{i j}, \\
b_{i j} & \geq c_{i j} \geq-b_{i j}, \\
c_{i j} & =\operatorname{tr}\left[\left(C^{\mathrm{Cl}}-C\right) T_{i}\left(\vec{x}_{j}\right)\right] .
\end{aligned}
$$

Combining this solution with the outer minimization, we finally have that $M(C)$ is half of the solution of

minimize: $a$

subject to: $a \geq s_{i}$,

$$
\begin{aligned}
& s_{i}=\sum_{j} b_{i j}, \\
& b_{i j} \geq c_{i j} \geq-b_{i j}, \\
& c_{i j}=\operatorname{tr}\left[\left(C^{\mathrm{Cl}}-C\right) T_{i}\left(\vec{x}_{j}\right)\right], \\
& C^{\mathrm{Cl}}=\sum_{y_{K}, \ldots, y_{1}} \mathbb{P}_{K}(\vec{y}) P_{y_{K}} \otimes \cdots \otimes P_{y_{1}}, \\
& \mathbb{P}_{K}(\vec{y}) \text { joint prob distribution, }
\end{aligned}
$$

which is a linear program.

In order to simplify the numerical implementation and the derivation of the dual program, we also order the 
vectors $\vec{y}$ (arbitrarily), identify $p_{k}$ with $\mathbb{P}_{K}(\vec{y}(k))$, and define $\alpha_{i j k}$,

$$
\operatorname{tr}\left[C^{\mathrm{Cl}} T_{i}\left(\vec{x}_{j}\right)\right]=\sum_{k} p_{k} \alpha_{i j k}
$$

for all $p_{k}$, i.e.,

$$
\alpha_{i j k}=\operatorname{tr}\left[P_{y_{K}(k)} \otimes \cdots \otimes P_{y_{1}(k)} T_{i}\left(\vec{x}_{j}\right)\right]
$$

for the sequence $y_{K}(k), \ldots, y_{1}(k)$ corresponding to $\vec{y}(k)$. In addition, we define

$$
\beta_{i j}=\operatorname{tr}\left[C T_{i}\left(\vec{x}_{j}\right)\right],
$$

which allows us to write

$$
\begin{aligned}
c_{i j} & =\operatorname{tr}\left[\left(C^{\mathrm{Cl}}-C\right) T_{i}\left(\vec{x}_{j}\right)\right] \\
& =\sum_{k} p_{k} \alpha_{i j k}-\beta_{i j} .
\end{aligned}
$$

Then, the above optimization problem is equivalent to

minimize: $a$

$$
\begin{aligned}
& \text { subject to: } \sum_{j} b_{i j}-a \leq 0, \\
& \sum_{k} p_{k} \alpha_{i j k}-\beta_{i j}-b_{i j} \leq 0, \\
& -\sum_{k} p_{k} \alpha_{i j k}+\beta_{i j}-b_{i j} \leq 0, \\
& \sum_{k} p_{k}-1=0, \\
& p_{k}, a, b_{i j} \geq 0 .
\end{aligned}
$$

The Lagrangian corresponding to this problem is

$$
\begin{aligned}
& L\left(a, p_{k}, b_{i j}, X_{i}, Y_{i j}, Z_{i j}, W\right) \\
& =a\left[1-\sum_{i} X_{i}\right]+\sum_{i j} b_{i j}\left[X_{i}-Y_{i j}-Z_{i j}\right] \\
& \quad+\sum_{k} p_{k}\left[\sum_{i j} \alpha_{i j k}\left(Y_{i j}-Z_{i j}\right)-W\right] \\
& \quad+W+\sum_{i j} \beta_{i j}\left(Z_{i j}-Y_{i j}\right),
\end{aligned}
$$

and the dual function is explicitly written

$$
\begin{aligned}
& q\left(X_{i}, Y_{i j}, Z_{i j}, W\right) \\
& \quad=\inf _{p_{k} \geq 0, a, b_{i j}} L\left(a, p_{k}, b_{i j}, X_{i}, Y_{i j}, Z_{i j}, W\right),
\end{aligned}
$$

where we used that $a, b_{i j} \geq 0$ is implicit in the remaining conditions. The dual problem is then given by

$$
\begin{aligned}
\text { maximize: } & W+\sum_{i j} \beta_{i j}\left(Z_{i j}-Y_{i j}\right) \\
\text { subject to: } & \sum_{i} X_{i}=1, \\
& X_{i}-Y_{i j}-Z_{i j}=0 \quad \forall i j, \\
& \sum_{i j} \alpha_{i j k}\left(Y_{i j}-Z_{i j}\right)-W \geq 0 \quad \forall k, \\
& X_{i}, Y_{i j}, Z_{i j} \geq 0, \\
& W \in \mathbb{R},
\end{aligned}
$$

which can straightforwardly be reformulated as

maximize: $\Omega$

$$
\begin{array}{cl}
\text { subject to: } & \Omega \leq \sum_{i j}\left(\alpha_{i j k}-\beta_{i j}\right)\left(2 Y_{i j}-X_{i}\right) \quad \forall k, \\
& \sum_{i} X_{i}=1, \\
& X_{i}, Y_{i j}, X_{i}-Y_{i j} \geq 0, \\
& \Omega \in \mathbb{R} .
\end{array}
$$

Evidently, the above considerations are amenable to many extensions, but that is a matter of future work.

\section{APPENDIX F: NON-DISCORD-CREATING MAPS}

Here, for comprehensiveness, we characterize the set of maps $\Gamma: \mathcal{B}\left(\mathcal{H}_{s}^{i} \otimes \mathcal{H}_{e}^{i}\right) \rightarrow \mathcal{B}\left(\mathcal{H}_{s}^{\circ} \otimes \mathcal{H}_{e}^{\circ}\right)$ that map discordzero states to discord-zero states, where we mean discord zero with respect to the classical basis. Such systemenvironment maps form a subset of the NDGD maps of Definition 3 [in the sense that a set of them would satisfy Eq. (68)] and would thus lead to classical statistics on the level of the system. However, for classical statistics, it is not necessary that the underlying maps do not create discord.

To facilitate notation, throughout this Appendix, we denote discord-zero states as DØ states and maps that do not create discord as $\mathrm{D} \emptyset$ maps. We have the following lemma:

Lemma 3. (Structure of $\mathrm{D} \emptyset$ maps) The Choi state $G$ of a DØ map $\Gamma: \mathcal{B}\left(\mathcal{H}_{s}^{i} \otimes \mathcal{H}_{e}^{i}\right) \rightarrow \mathcal{B}\left(\mathcal{H}_{s}^{\circ} \otimes \mathcal{H}_{e}^{\circ}\right)$ is of the form

$$
G=\sum_{k, j=1}^{d_{s}} p_{k \mid j} \Pi_{k}^{\circ} \otimes \Pi_{j}^{i} \otimes O_{j k}^{\circ i}+G^{\perp},
$$

where $\left\{\Pi_{l}^{\mathrm{i} / \circ}\right\}$ are orthogonal rank-1 projectors on $\mathcal{H}_{s}^{\mathrm{i} / \circ}$ that are diagonal in the computational basis, $O_{j k}^{\circ i} \in$ $\mathcal{B}\left(\mathcal{H}_{e}^{\circ} \otimes \mathcal{H}_{e}^{i}\right)$ is the Choi state of a CPTP map $\Omega_{j k}: \mathcal{B}\left(\mathcal{H}_{e}^{i}\right) \rightarrow \mathcal{B}\left(\mathcal{H}_{e}^{\circ}\right), p_{k \mid j}$ is a conditional probability distribution, i.e., $\sum_{k} p_{k \mid j}=1$ and $p_{k \mid j} \geq 0$, and $G^{\perp} \in$ $\mathcal{B}\left(\mathcal{H}_{s}^{\circ} \otimes \mathcal{H}_{e}^{\circ} \otimes \mathcal{H}_{s}^{i} \otimes \mathcal{H}_{e}^{i}\right)$ is orthogonal to the set of 
$\mathrm{D} \varnothing$ states, i.e., $\operatorname{tr}\left[(\mathbb{1} \otimes \rho) G^{\perp}\right]=0$ for all $\mathrm{D} \emptyset$ states $\rho \in \mathcal{B}\left(\mathcal{H}_{s}^{\mathrm{i}} \otimes \mathcal{H}_{e}^{\mathrm{i}}\right)$.

Before we prove this lemma, we emphasize its structural relation to the representation of MIOs, i.e., the structure of maps $\mathcal{F}: \mathcal{B}\left(\mathcal{H}_{s}^{\mathrm{i}}\right) \rightarrow \mathcal{B}\left(\mathcal{H}_{s}^{\circ}\right)$ that map incoherent states $\rho \in$ $\Xi \subset \mathcal{B}\left(\mathcal{H}_{s}^{\mathrm{i}}\right)$ onto incoherent states $\rho^{\prime}=\mathcal{F}[\rho] \in \Xi \subset \mathcal{B}\left(\mathcal{H}_{s}^{\circ}\right)$, where $\Xi$ denotes the set of incoherent states with respect to the computational basis. The Choi state $F$ of the map $\mathcal{F}$ is a positive element of $\mathcal{B}\left(\mathcal{H}_{s}^{\circ} \otimes \mathcal{H}_{s}^{i}\right)$. Choosing a basis $\left\{\tau_{k}^{\circ}\right\}_{k=1}^{d_{s}^{2}}$ and $\left\{\omega_{j}^{\mathrm{i}}\right\}_{j=1}^{d_{s}^{2}}$ for $\mathcal{B}\left(\mathcal{H}_{s}^{\circ}\right)$ and $\mathcal{B}\left(\mathcal{H}_{s}^{\mathrm{i}}\right)$, respectively, any $F$ can be written as

$$
F=\sum_{j, k} f_{j k} \tau_{k}^{\circ} \otimes \omega_{j}^{i},
$$

where $f_{j k} \in \mathbb{R}$. We can choose the basis $\left\{\omega_{j}^{i}\right\}$ to consist of the $d_{s}$ rank-1 projectors $\Pi_{j}^{i}$ in the computational basis and $d_{s}\left(d_{s}-1\right)$ elements $\tilde{\Pi}_{s}^{\mathrm{i}}$ that are orthogonal to these projectors, i.e., such that $\operatorname{tr}\left(\Pi_{j}^{i} \tilde{\Pi}_{s}^{i}\right)=0$ [e.g., one could choose the off-diagonal elements $|m\rangle\langle n|+| n\rangle\langle m|$ and $₫(|m\rangle\langle n|-$ $|n\rangle\langle m|)]$. With this choice of basis elements, Eq. (F2) reads

$$
F=\sum_{j, k} f_{k j} \tau_{k}^{\circ} \otimes \Pi_{j}^{i}+\sum_{r, s} \tilde{f}_{r s} \tau_{r}^{\circ} \otimes \tilde{\Pi}_{s}^{i} .
$$

Imposing the requirement that $\mathcal{F}$ does not create coherences with respect to the classical basis then yields

$$
F=\sum_{j, k} p_{k \mid j} \Pi_{k}^{\circ} \otimes \Pi_{j}^{\mathrm{i}}+\sum_{r, s} \tilde{f}_{r s} \tau_{r}^{\circ} \otimes \tilde{\Pi}_{s}^{\mathrm{i}},
$$

where $p_{k \mid j} \geq 0, \sum_{k} p_{k \mid j}=1$, and $\tau_{r}^{\circ} \in \mathcal{B}\left(\mathcal{H}_{s}^{\circ}\right)$. Indeed, an $F$ of the form of Eq. (F4) yields an incoherent output state for any incoherent input state $\rho_{\mathrm{cl}}=\sum_{r=1}^{d_{s}} q_{r} \Pi_{r}^{i} \in \Xi$ :

$$
\mathcal{F}\left[\rho_{\mathrm{cl}}\right]=\operatorname{tr}_{\dot{i}}\left[\left(\mathbb{1}^{\circ} \otimes \rho_{\mathrm{cl}}^{\mathrm{T}}\right) F\right]=\sum_{k r} p_{k \mid r} q_{r} \Pi_{k}^{\circ} .
$$

Importantly, Eq. (F4) constitutes a decomposition of the form $F=F^{\|}+F^{\perp}$, where $F^{\|}=\sum_{j, k} p_{k \mid j} \Pi_{k}^{\circ} \otimes \Pi_{j}^{i}$ encapsulates the action of $\mathcal{F}$ on incoherent states, and $F^{\perp}$ is such that all incoherent states lie in its kernel, i.e., $\operatorname{tr}\left(\rho F^{\perp}\right)=0$ for all $\rho \in \Xi$. The fact that $F^{\perp}$ does not have to vanish in order for $\mathcal{F}$ to be a MIO demonstrates, in a transparent way, the (wellknown) fact that there are MIOs that necessitate coherent resources for their implementation [5,15,17].

As emphasized throughout the main body of this paper, $\mathrm{D} \emptyset$ states reduce to incoherent ones when the environment is trivial. Consequently, $\mathrm{D} \varnothing$ maps are the natural extension of MIOs, and the proof of Lemma 3 follows similar logic to the above proof for the structural properties of MIOs:

Proof.-Employing the reasoning that led to Eq. (F4), any DØ map $\Gamma$ has a Choi state $G$ of the form

$$
\begin{aligned}
G= & \sum_{k j \mu \nu} g_{k \mu j \nu} \tau_{k}^{\circ} \otimes \Pi_{j}^{\mathrm{i}} \otimes N_{\mu \nu}^{\circ \mathrm{i}} \\
& +\sum_{r s \mu \nu} \tilde{g}_{r \mu s \nu} \tau_{r}^{\circ} \otimes \tilde{\Pi}_{s}^{\mathrm{i}} \otimes N_{\mu \nu}^{\circ i},
\end{aligned}
$$

where $g_{k \mu j \nu}, \tilde{g}_{r \mu s \nu} \in \mathbb{R}$ and $\left\{N_{\mu \nu}^{\circ i}\right\}_{\mu, \nu=1}^{d_{e}^{2}}$ is a basis of $\mathcal{B}\left(\mathcal{H}_{e}^{\circ} \otimes \mathcal{H}_{e}^{\mathrm{i}}\right)$. As for the case of MIOs, Eq. (F6) constitutes a decomposition $G=G^{\|}+G^{\perp}$, where $G^{\perp}$ is orthogonal to the set of D $\varnothing$ states. Consequently, the action of $\Gamma$ on any $\mathrm{D} \varnothing$ state is entirely encapsulated in $G^{\|}$, and it remains to show that this term is of the form given in the lemma. To this end, we note that a map $\Gamma$ is DØ iff it maps any state of the form $\Pi_{\ell}^{i} \otimes \eta_{\ell}^{i}$ to a $\mathrm{D} \varnothing$ state. Letting $\Gamma$ act on such a product state, we obtain

$$
\begin{aligned}
\Gamma\left[\Pi_{\ell}^{\mathrm{i}} \otimes \eta_{\ell}^{\mathrm{i}}\right] & =\operatorname{tr}_{\dot{i}}\left\{\left[\mathbb{1}^{\circ} \otimes\left(\Pi_{\ell}^{\mathrm{i}} \otimes \eta_{\ell}^{\mathrm{i}}\right) \mathrm{T}\right] G^{\|}\right\} \\
& =\sum_{k \mu \nu} g_{k \mu \ell \nu} \tau_{k}^{\circ} \otimes \operatorname{tr}_{\mathrm{i}}\left[\left(\mathbb{1}^{\circ} \otimes \eta_{\ell}^{\mathrm{i} \mathrm{T}}\right) N_{\mu \nu}^{\circ \mathrm{i}}\right] \\
& \stackrel{!}{=} \sum_{r} p_{r \mid \ell} \Pi_{t}^{\circ} \otimes \xi_{r \mid \ell}^{\circ},
\end{aligned}
$$

where $\sum_{r} p_{r \mid \ell}=1$ and $p_{r \mid \ell} \geq 0$, and $\xi_{r}^{\circ} \in \mathcal{B}\left(\mathcal{H}_{e}^{\circ}\right)$ are states of the environment. The last line of Eq. (F8) stems from the requirement that $\Gamma$ is a $D \emptyset$ map, and the remaining open index $\ell$ signifies that the resulting output state depends on the input state $\Pi_{\ell}^{i} \otimes \eta_{\ell}^{i}$. In the same way as above, we can choose the basis $\left\{\tau_{k}^{\circ}\right\}$ to consist of projectors $\left\{\Pi_{k}^{\circ}\right\}$ onto the computational basis and elements that are orthogonal to these projectors. Then, comparing Eqs. (F7) and (F8), we see that all of the terms of $G^{\|}$, where $\tau_{k}^{\circ}$ is not a projector onto the computational basis, must vanish. Finally, the terms $N_{\mu \nu}^{\circ i}$ have to be such that $\operatorname{tr}_{\mathrm{i}}\left[\left(\mathbb{1} \otimes \eta_{l}^{\mathrm{iT}}\right) \sum_{\mu \nu} g_{k \mu l \nu} N_{\mu \nu}^{\mathrm{oi}}\right]$ yields the correct output state $p_{k \mid \ell} \xi_{k \mid \ell}^{\circ}$. Consequently, $\sum_{\mu \nu} g_{k \mu l \nu} N_{\mu \nu}^{\circ i}$ can be chosen to be (up to normalization $p_{k \mid \ell}$ ) the Choi state $O_{k \ell}^{\circ i}$ of a CPTP map. Putting these observations together yields Eq. (F1).

\section{APPENDIX G: PROOF THAT NDGD $\Rightarrow$ CLASSICAL PROCESS}

For the proof of Theorem 3, we employ the fact that the completely dephasing map has no influence on the outcomes of a measurement in the computational basis, i.e.,

$$
\mathcal{P}_{x_{j}}=\Delta_{j} \circ \mathcal{P}_{x_{j}}=\mathcal{P}_{x_{j}} \circ \Delta_{j} \quad \forall x_{j} .
$$

The probability $\mathbb{P}_{k}\left(x_{k}, \ldots, x_{1}\right)$ to measure outcomes $\left\{x_{k}, \ldots, x_{1}\right\}$ at times $\left\{t_{k}, \ldots, t_{1}\right\}$ is given by [see Eq. (66)]

$$
\operatorname{tr}\left\{\left(\mathcal{P}_{x_{k}} \otimes \mathcal{I}_{k}^{e}\right) \circ \cdots \circ \Gamma_{t_{2}, t_{1}} \circ\left(\mathcal{P}_{x_{1}} \otimes \mathcal{I}_{1}^{e}\right)\left[\eta_{t_{1}}^{s e}\right]\right\},
$$

where $\left\{\Gamma_{t_{j}, t_{j-1}}\right\}$ are system-environment CPTP maps and $\eta_{t_{1}}^{s e}$ is the system-environment state at time $t_{1}$. Summing 
this probability distribution over the outcomes at time $t_{j}$ amounts to replacing $\mathcal{P}_{x_{j}}$ in Eq. (G2) by $\Delta_{j}$. "Zooming in" on the relevant time (and leaving the $\mathcal{I}^{e}$ implicit), we see that

$$
\begin{aligned}
& \mathcal{P}_{x_{j+1}} \circ \Gamma_{t_{j+1}, t_{j}} \circ \Delta_{j} \circ \Gamma_{t_{j}, t_{j-1}} \circ \mathcal{P}_{x_{j-1}} \\
& =\mathcal{P}_{x_{j+1}} \circ \Delta_{j+1} \circ \Gamma_{t_{j+1}, t_{j}} \circ \Delta_{j} \circ \Gamma_{t_{j}, t_{j-1}} \circ \Delta_{j-1} \circ \mathcal{P}_{x_{j-1}} \\
& =\mathcal{P}_{x_{j+1}} \circ \Gamma_{t_{j+1}, t_{j}} \circ \mathcal{I}_{j} \circ \Gamma_{t_{j}, t_{j-1}} \circ \mathcal{P}_{x_{j-1}},
\end{aligned}
$$

where we have used Eq. (G1) in the first line and both the fact that the dynamics is NDGD and Eq. (G1) in the second line. As Eq. (G3) holds for arbitrary times $t_{j}$, it implies that for NDGD dynamics, the completely dephasing map cannot be distinguished from the identity map when the process is probed by measurements in the computational basis, which implies that the Kolmogorov condition holds for any joint probabilities with at least three different times. For the two-time joint probabilities, we can exploit, along with the NDGD property, the fact that the initial state has zero discord. We have

$$
\begin{aligned}
& \sum_{x_{1}} \mathbb{P}_{2}\left(x_{2}, x_{1}\right) \\
& =\operatorname{tr}\left\{\mathcal{P}_{x_{2}} \circ \Gamma_{t_{2}, t_{1}} \circ \Delta_{1} \circ \Gamma_{t_{1}, t_{0}}\left[\eta_{t_{0}}^{s e}\right]\right\} \\
& =\operatorname{tr}\left\{\mathcal{P}_{x_{2}} \circ \Delta_{2} \circ \Gamma_{t_{2}, t_{1}} \circ \Delta_{1} \circ \Gamma_{t_{1}, t_{0}}\left[\eta_{t_{0}}^{s e}\right]\right\} \\
& =\operatorname{tr}\left\{\mathcal{P}_{x_{2}} \circ \Delta_{2} \circ \Gamma_{t_{2}, t_{1}} \circ \Delta_{1} \circ \Gamma_{t_{1}, t_{0}} \circ \Delta_{0}\left[\eta_{t_{0}}^{s e}\right]\right\} \\
& =\operatorname{tr}\left\{\mathcal{P}_{x_{2}} \circ \Gamma_{t_{2}, t_{0}}\left[\eta_{t_{0}}^{s e}\right]\right\}=\mathbb{P}_{1}\left(x_{2}\right),
\end{aligned}
$$

where we used Eq. (G2) and $\sum_{x_{1}} \mathcal{P}_{x_{1}}=\Delta_{1}$ in the first line, Eq. (G1) in the second line, the invariance of the initial zero-discord state with respect to $\Delta_{0}$ in the third line, and finally, the definition of NDGD dynamics, Eq. (G1), and the invariance of $\eta_{t_{0}}^{s e}$ in the fourth line. Consequently, the resulting statistics satisfy all of the Kolmogorov conditions and are thus classical.

\section{APPENDIX H: CLASSICAL ITY $\not$ NDGD}

Here, we provide an example of dynamics that are not NDGD yet lead to classical dynamics, thus demonstrating that it is not necessary for a dynamics to be NDGD in order for it to appear classical. We consider the following situation (see Fig. 14 for a graphical representation): Let the system of interest be a qubit that is initially in state $|0\rangle$, and let the initial environment be in a plus state, i.e., $\tau_{t_{0}}^{e}=\frac{1}{\sqrt{2}}(|0\rangle+|1\rangle)$. The first evolution $\Gamma_{t_{1}, t_{0}}$ from $t_{0}$ to $t_{1}$ is a CNOT gate, such that the system-environment state at $t_{1}$ is a maximally entangled state. The second evolution $\Gamma_{t_{2}, t_{1}}$ from $t_{1}$ to $t_{2}$ is such that it yields a system-environment state $\mathbb{1}_{s} / 2 \otimes|0\rangle\langle 0|$ if the $s e^{\prime}$ input state is $\varphi_{s e^{\prime}}^{+}$, and $\mathbb{1}_{s} / 2 \otimes$ $|1\rangle\langle 1|$ otherwise. Consequently, when the completely dephasing map is applied at $t_{1}$, the system-environment

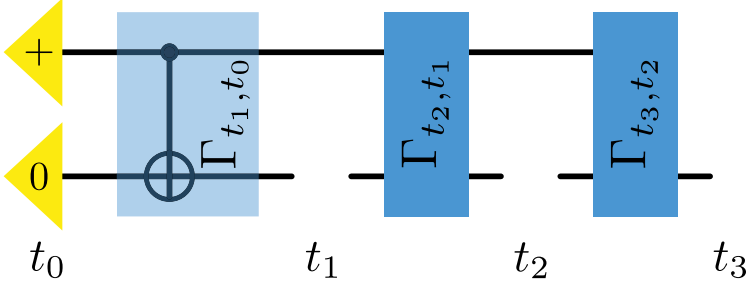

FIG. 14. Non-NDGD dynamics that leads to classical statistics. The first map $\Gamma_{t_{1}, t_{0}}$ (blue transparent box) performs a CNOT gate on the system and the environment. The subsequent CPTP map $\Gamma_{t_{2}, t_{1}}$ maps $\varphi^{+}$and $\mathbb{1} / 4$ onto two different system-environment states with the same reduced system state $\rho_{t_{3}}=\mathbb{1} / 2$. The final CPTP map $\Gamma_{t_{3}, t_{2}}$ is such that it induces a unital dynamics on the system. Consequently, the system state at $t_{1}, t_{2}$, and $t_{3}$ is maximally mixed independent of whether the completely dephasing or the identity map was implemented at $t_{1}$ and $t_{2}$.

state at $t_{2}$ is $\mathbb{1}_{s} / 2 \otimes \mathbb{1}_{e} / 2$, while it is equal to $\mathbb{1}_{s} / 2 \otimes|0\rangle\langle 0|$ if the identity map was implemented, and as such, the dynamics is not NDGD. However, the system state is always maximally mixed, independent of whether $\Delta_{1}$ or $\mathcal{I}_{1}$ was implemented at time $t_{1}$. To make the example nontrivial, we add a third free dynamics $\Gamma_{t_{3}, t_{2}}$ from $t_{2}$ to $t_{3}$. We choose $\Gamma_{t_{3}, t_{2}}$ such that it induces a unital dynamics on the level of the system, independent of the environment state at $t_{2}$. This result happens, e.g., when the corresponding system-environment Hamiltonian is of product form, i.e., $H_{s e}=H_{s} \otimes H_{e}$, independent of the explicit form of the respective terms [76]. With this final dynamics, the system state at each of the times $t_{1}, t_{2}$, and $t_{3}$ is maximally mixed, and the resulting statistics satisfy Kolmogorov conditions; i.e., they are classical.

\section{APPENDIX I: EXAMPLE OF A GENUINELY QUANTUM PROCESS}

Consider the following process, depicted in Fig. 15, which is a variation on that presented in Sec. VII. The process begins with a two-qubit system-environment state in the Bell pair $\varphi_{s e}^{+}$, the system part of which the experimenter has access to measure at $t_{1}$. Next, the process performs the CPTP system-environment map $\Gamma_{z}^{s e z}: \mathcal{B}\left(\mathcal{H}^{s^{i}} \otimes \mathcal{H}^{e^{i}}\right) \rightarrow$ $\mathcal{B}\left(\mathcal{H}^{s^{\circ}} \otimes \mathcal{H}^{e^{\circ}} \otimes \mathcal{H}^{z^{\circ}}\right)$, whose action is as follows: It measures its joint inputs in the Bell basis, and if the measurement outcome corresponds to $\varphi_{s e}^{+}$, it outputs a $\varphi_{s e}^{+}$systemenvironment state as well as a classical flag state $|0\rangle_{z}$; on the other hand, if the measurement outcome does not correspond to $\varphi_{s e}^{+}$, it outputs a system-environment state whose system part is a pure state in the $z$ basis and sets the flag state to $|1\rangle_{z}$ to indicate that the system state has been biased in the $z$ basis. Thus, the action of the map is as follows:

$$
\begin{aligned}
\Gamma_{z}^{\operatorname{sez}}\left[\eta_{s e}\right]= & \operatorname{tr}\left[\varphi_{s e}^{+} \eta_{s e}\right] \varphi_{s e}^{+} \otimes|0\rangle\left\langle\left. 0\right|_{z}\right. \\
& +\operatorname{tr}\left[\left(\mathbb{1}_{s e}-\varphi_{s e}^{+}\right) \eta_{s e}\right]|0\rangle\left\langle\left. 0\right|_{s} \otimes \tau_{e} \otimes \mid 1\right\rangle\left\langle\left. 1\right|_{z} .\right.
\end{aligned}
$$




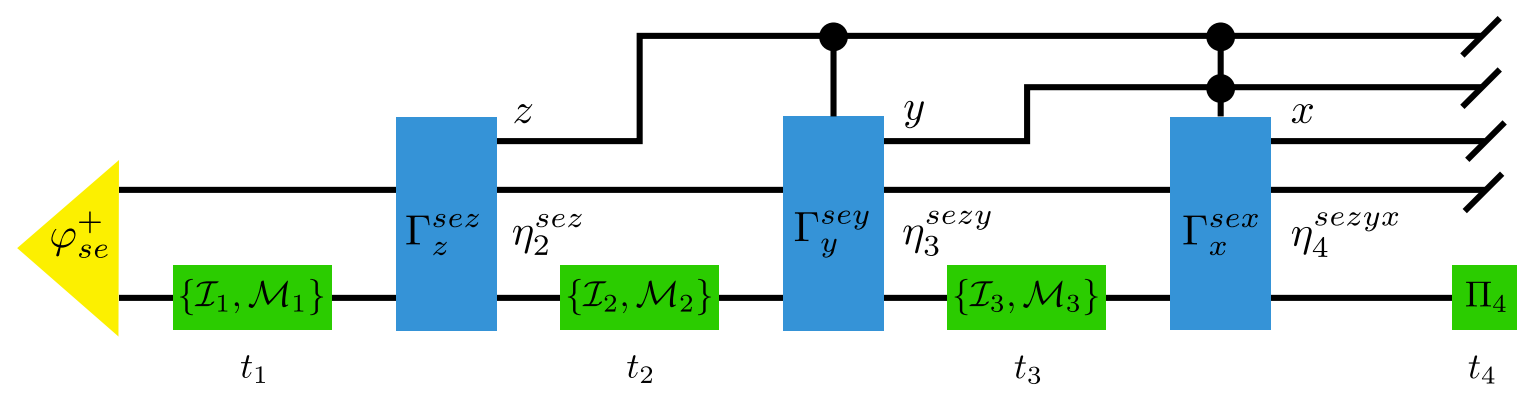

FIG. 15. Genuinely quantum process. The system and environment begin in a maximally entangled Bell state $\varphi_{s e}^{+}$. As described in the text, the process dynamics consists of a sequence of maps, $\Gamma_{z}^{s e z}, \Gamma_{y}^{s e y}, \Gamma_{x}^{s e x}$, that either output $\varphi_{s e}^{+}$or else bias the system in either the $z, y$, or $x$ basis, respectively [see Eqs. (I1)-(I3)]. The overall implementation of each of these maps is controlled on the joint state of all previous classical flag states $z, y, x$, which encode whether or not the system has already been biased. We show that this process is genuinely quantum by tracking the system-environment state throughout the dynamics, conditioned on whether the identity map $\mathcal{I}$ or an arbitrary CPTP map $\mathcal{M}_{i}$ was implemented at time $t_{i}$; the labels $\eta_{2}^{\text {sez }}, \eta_{3}^{\text {sezy }}$, and $\eta_{4}^{\text {sezyx }}$ refer to the overall joint state immediately prior to the interrogation at the relevant time [see Eqs. (I4), (I6), (I8), (I9), (I11), and (I12)]. In particular, we show that there does not exist a nonpathological POVM $\Pi_{4}$ that an experimenter can implement at $t_{4}$ such that the four sequences $\left\{\mathcal{I}_{1}, \mathcal{I}_{2}, \mathcal{I}_{3}\right\},\left\{\mathcal{M}_{1}, \mathcal{I}_{2}, \mathcal{I}_{3}\right\}$, $\left\{\mathcal{I}_{1}, \mathcal{M}_{2}, \mathcal{I}_{3}\right\}$, and $\left\{\mathcal{I}_{1}, \mathcal{I}_{2}, \mathcal{M}_{3}\right\}$ cannot be distinguished, thereby proving that the process is genuinely quantum.

For this map (and all that follow in this example), the output state of the environment when the $\varphi_{s e}^{+}$outcome is not recorded is irrelevant for our argument; as such, we simply write a generic quantum state $\tau_{e}$.

Following this part of the dynamics, the experimenter has access to measure the system at time $t_{2}$. The subsequent dynamics of the process is controlled on the state of the classical $z$ flag: If it is in the state $|0\rangle_{z}$, the systemenvironment state is subject to a similar dynamics as before, $\Gamma_{y}^{\text {sey }}: \mathcal{B}\left(\mathcal{H}^{s^{i}} \otimes \mathcal{H}^{e^{i}}\right) \rightarrow \mathcal{B}\left(\mathcal{H}^{s^{\circ}} \otimes \mathcal{H}^{e^{\circ}} \otimes \mathcal{H}^{y^{\circ}}\right) ; \quad$ however, this time, if the Bell basis measurement outcome does not correspond to $\varphi_{s e}^{+}$, the system is biased in the $y$ basis, e.g., set to the -1 eigenstate of $\sigma^{(y)},\left|-^{(y)}\right\rangle:=\frac{1}{\sqrt{2}}(|0\rangle-i|1\rangle)$, with a classical $y$ flag set to the state $|1\rangle_{y}$ and sent forward. If, on the other hand, the $z$ flag is in the state $|1\rangle_{z}$, the systemenvironment state undergoes trivial dynamics (i.e., is subject to the identity map), and the $y$ flag is set to $|0\rangle_{y}$. In either case, the previous $z$ flag state is also sent forward unperturbed. Thus, between $t_{2}$ and $t_{3}$, the system-environment state evolves conditionally according to

$$
\begin{aligned}
& \begin{array}{l}
z=0: \\
: \Gamma_{y}^{s e y}\left[\eta_{s e}\right]=\operatorname{tr}\left[\varphi_{s e}^{+} \eta_{s e}\right] \varphi_{s e}^{+} \otimes|0\rangle\left\langle\left. 0\right|_{y}\right. \\
+\operatorname{tr}\left[\left(\mathbb{1}_{s e}-\varphi_{s e}^{+}\right) \eta_{s e}\right]\left|-^{(y)}\right\rangle\left\langle-\left.^{(y)}\right|_{s} \otimes \tau_{e} \otimes \mid 1\right\rangle\left\langle\left. 1\right|_{y},\right.
\end{array} \\
& \underline{z=1:}: \mathcal{I}^{s e}\left[\eta_{s e}\right] \otimes|0\rangle\left\langle\left. 0\right|_{y} .\right.
\end{aligned}
$$

Then, the experimenter has access to the system at $t_{3}$.

The final portion of the dynamics between $t_{3}$ and $t_{4}$ follows a similar construction as above, but the implementation of the map $\Gamma_{x}^{s e x}: \mathcal{B}\left(\mathcal{H}^{s^{1}} \otimes \mathcal{H}^{e^{i}}\right) \rightarrow \mathcal{B}\left(\mathcal{H}^{s^{0}} \otimes\right.$ $\left.\mathcal{H}^{e^{\circ}} \otimes \mathcal{H}^{x^{\circ}}\right)$ is controlled on the joint state of the $z$ and $y$ classical flags. If $z y=00$, the system-environment state is measured in the Bell basis: If the measurement outcome does not correspond to $\varphi_{s e}^{+}$, the system is biased in the $x$ basis, e.g., set to the -1 eigenstate of $\sigma^{(x)}$, $\left|-^{(x)}\right\rangle:=\frac{1}{\sqrt{2}}(|0\rangle-|1\rangle)$, with a classical $x$ flag set to the state $|1\rangle_{x}$ and sent forward. If, on the other hand, $z y \neq 00$, system and environment undergo trivial dynamics (i.e., is subject to the identity map), and the $x$ flag is set to $|0\rangle_{x}$. Mathematically, the controlled dynamics is described as

$$
\begin{gathered}
\begin{array}{c}
z y=00: \Gamma_{x}^{s e x}\left[\eta_{s e}\right]=\operatorname{tr}\left[\varphi_{s e}^{+} \eta_{s e}\right] \varphi_{s e}^{+} \otimes|0\rangle\left\langle\left. 0\right|_{x}\right. \\
+\operatorname{tr}\left[\left(\mathbb{1}_{s e}-\varphi_{s e}^{+}\right) \eta_{s e}\right]\left|-{ }^{(x)}\right\rangle\left\langle-\left.^{(x)}\right|_{s}\right. \\
\otimes \tau_{e} \otimes|1\rangle\left\langle\left. 1\right|_{x},\right.
\end{array} \\
\underline{z y=10,01: \mathcal{I}^{s e}\left[\eta_{s e}\right] \otimes|0\rangle\left\langle\left. 0\right|_{x} .\right.}
\end{gathered}
$$

Note that the flag state $z y=11$ cannot occur. Finally, the environment and all flag states are discarded, and the experimenter has access to the system at $t_{4}$, concluding the process.

We now show that there exists no (nonpathological) unrestricted measurement scheme for this process such that the statistics observed are classical; i.e., we prove that the process is genuinely quantum. As in the main text, we achieve this goal by considering the state of the system to be measured at the final time $t_{4}$ conditioned on a history of identity maps and arbitrary CPTP maps $\left\{\mathcal{M}_{1}, \mathcal{M}_{2}, \mathcal{M}_{3}\right\}$ implemented at various sets of earlier times. In each case, by demanding classicality, we end up with a different constraint on the structure of the POVM required such that no invasiveness can be detected at the final time, and the only valid POVMs that simultaneously satisfy all conditions are the pathological ones that do not reveal anything about the process. The conclusion is that any nonpathological POVM at $t_{4}$ will be able to distinguish between previous implementations of the identity map or an arbitrary nonpathological instrument at a given time, therefore picking up on the invasiveness of (at least some of) 
the previous interrogations and leading to nonclassical statistics.

Consider first the scenario where the experimenter implements identity maps at the first three times, $\mathcal{I}_{1}, \mathcal{I}_{2}$, $\mathcal{I}_{3}$. In this case, the overall state immediately prior to the measurement at $t_{4}$ is

$$
\eta_{4}^{\text {sezyx }}\left(\mathcal{I}_{1}, \mathcal{I}_{2}, \mathcal{I}_{3}\right)=\varphi_{s e}^{+} \otimes|000\rangle\left\langle\left. 000\right|_{z y x} .\right.
$$

The reduced system state is then maximally mixed:

$$
\eta_{4}^{s}\left(\mathcal{I}_{1}, \mathcal{I}_{2}, \mathcal{I}_{3}\right)=\frac{\mathbb{1}}{2}
$$

Next, consider the case where the experimenter implements the identity map at the first two times, $\mathcal{I}_{1}, \mathcal{I}_{2}$, followed by an arbitrary CPTP map $\mathcal{M}_{3} \neq \mathcal{I}_{3}$ at $t_{3}$. The systemenvironment joint state immediately prior to $t_{3}$ is $\varphi_{s e}^{+}$since the previous identity maps on the system and the dynamics $\Gamma_{z}^{s e z}, \Gamma_{y}^{s e y}$ leading up to $t_{3}$ preserve the initial state; moreover, the $z y$ flag is in the joint state 00 since both previous Bell basis measurements are necessarily successful. Now, the system-local CPTP map $\mathcal{M}_{3} \neq \mathcal{I}_{3}$ will perturb the joint system-environment state, so the map $\Gamma_{x}^{s e x}$ (which is implemented due to the joint state of the input flags) only successfully records the outcome corresponding to $\varphi_{s e}^{+}$with some probability $r=\operatorname{tr}\left[\varphi_{s e}^{+}\left(\mathcal{M}_{3}^{s} \otimes \mathcal{I}^{e}\right)\left[\varphi_{s e}^{+}\right]\right]<1$; otherwise, the system is biased in the $x$ basis. The total joint state immediately prior to $t_{4}$ in this scenario is then

$$
\begin{aligned}
& \eta_{4}^{\text {sezyx }}\left(\mathcal{I}_{1}, \mathcal{I}_{2}, \mathcal{M}_{3}\right) \\
& =r \varphi_{s e}^{+} \otimes|000\rangle\left\langle\left. 000\right|_{z y x}\right. \\
& \quad+(1-r)\left|-^{(x)}\right\rangle\left\langle-\left.^{(x)}\right|_{s} \otimes \tau_{e} \otimes \mid 001\right\rangle\left\langle\left. 001\right|_{z y x} .\right.
\end{aligned}
$$

The reduced system state is thus biased in the $x$ basis:

$$
\eta_{4}^{s}\left(\mathcal{I}_{1}, \mathcal{I}_{2}, \mathcal{M}_{3}\right)=\frac{r}{2} \mathbb{1}+(1-r)\left|-{ }^{(x)}\right\rangle\left\langle-^{(x)}\right| .
$$

Next, consider the case where the experimenter implements the identity map at the first and third times, $\mathcal{I}_{1}, \mathcal{I}_{3}$, with an arbitrary CPTP map $\mathcal{M}_{2} \neq \mathcal{I}_{2}$ implemented in between at time $t_{2}$. The system-environment joint state immediately prior to $t_{2}$ is $\varphi_{s e}^{+}$since the previous identity map on the system and the dynamics $\Gamma_{z}^{\text {sez }}$ prior to $t_{2}$ again preserve the initial state; moreover, the $z$ flag is in the state 0 since the earlier Bell basis measurement is necessarily successful. Again, the system-local CPTP map $\mathcal{M}_{2} \neq \mathcal{I}_{2}$ will perturb the joint system-environment state, so the map $\Gamma_{y}^{\text {sey }}$ (which is implemented due to the state of the input flag) only successfully records the outcome corresponding to $\varphi_{s e}^{+}$with some probability $q=\operatorname{tr}\left[\varphi_{s e}^{+}\left(\mathcal{M}_{2}^{s} \otimes \mathcal{I}^{e}\right)\left[\varphi_{s e}^{+}\right]\right]<1 ; \quad$ otherwise, the system is biased in the $y$ basis. The total joint state immediately prior to $t_{3}$ in this scenario is then

$$
\begin{aligned}
\eta_{3}^{\text {sezy }}\left(\mathcal{I}_{1}, \mathcal{M}_{2}\right)= & q \varphi_{s e}^{+} \otimes|00\rangle\left\langle\left. 00\right|_{z y}\right. \\
& +(1-q)\left|-^{(y)}\right\rangle\left\langle-\left.^{(y)}\right|_{s} \otimes \tau_{e} \otimes \mid 01\right\rangle\left\langle\left. 01\right|_{z y} .\right.
\end{aligned}
$$

In this case, the experimenter then implements the identity map to the system at $t_{3}$, which leaves the overall state invariant. The subsequent system-environment dynamics $\Gamma_{x}^{s e x}$ will be enacted when $z y=00$, i.e., with probability $q$ : in each such run, the system-environment state is guaranteed to be in the state $\varphi_{s e}^{+}$; thus, the system-environment state output by $\Gamma_{x}^{s e x}$ will be also. In the other cases, when $z y \neq 00$, the subsequent dynamics will be trivial. Thus, the total joint state immediately prior to $t_{4}$ in this scenario is

$$
\begin{aligned}
\eta_{4}^{\text {sezyx }} & \left(\mathcal{I}_{1}, \mathcal{M}_{2}, \mathcal{I}_{3}\right) \\
= & q \varphi_{s e}^{+} \otimes|000\rangle\left\langle\left. 000\right|_{z y x}\right. \\
& +(1-q)\left|-^{(y)}\right\rangle\left\langle-\left.^{(y)}\right|_{s} \otimes \tau_{e} \otimes \mid 010\right\rangle\left\langle\left. 010\right|_{z y x} .\right.
\end{aligned}
$$

The final reduced system state is thus biased in the $y$ basis:

$$
\eta_{4}^{s}\left(\mathcal{I}_{1}, \mathcal{M}_{2}, \mathcal{I}_{3}\right)=\frac{q}{2} \mathbb{1}+(1-q)\left|-^{(y)}\right\rangle\left\langle-^{(y)}\right| .
$$

Lastly, consider the scenario where the experimenter first implements an arbitrary CPTP map $\mathcal{M}_{1} \neq \mathcal{I}_{1}$ at $t_{1}$, followed by identity maps at the second and third times, $\mathcal{I}_{2}, \mathcal{I}_{3}$. Just as in the main text, $\mathcal{M}_{1} \neq \mathcal{I}_{1}$ will perturb the initial system-environment state, so the map $\Gamma_{z}^{s e z}$ will only successfully record the outcome corresponding to $\varphi_{s e}^{+}$with some probability $p=\operatorname{tr}\left[\varphi_{s e}^{+}\left(\mathcal{M}_{1}^{s} \otimes \mathcal{I}^{e}\right)\left[\varphi_{s e}^{+}\right]\right]<1$; otherwise, the system will be biased in the $z$ basis. The total joint state immediately prior to $t_{2}$ in this scenario is then

$$
\begin{aligned}
\eta_{2}^{\text {sez }}\left(\mathcal{M}_{1}\right)= & p \varphi_{s e}^{+} \otimes|0\rangle\left\langle\left. 0\right|_{z}\right. \\
& +(1-p)|0\rangle\left\langle\left. 0\right|_{s} \otimes \tau_{e} \otimes \mid 1\right\rangle\left\langle\left. 1\right|_{z} .\right.
\end{aligned}
$$

The identity map implemented by the experimenter on the system at $t_{2}$ does not change this state. Thus, $\Gamma_{y}^{\text {sey }}$ will subsequently be enacted with probability $p$, i.e., when $z=0$. In such cases, the system-environment state is $\varphi_{s e}^{+}$, and the output of the map $\Gamma_{y}^{\text {sey }}$ will be also, accompanied by the classical $y$ flag with the value 0 . In the other cases, system and environment undergo trivial dynamics. Again, at $t_{3}$, implementation of the identity map on the system leaves the joint state unperturbed. Only when the joint state of $z y$ is 00 will the map $\Gamma_{x}^{\text {sex }}$ be implemented. In each such run, the system-environment state is guaranteed to be $\varphi_{s e}^{+}$, and thus so, too, will be the output of the map. In the other cases, trivial dynamics ensues. The overall joint state in this scenario immediately prior to $t_{4}$ is then 


$$
\begin{aligned}
\eta_{4}^{\text {sezyx }}\left(\mathcal{M}_{1}, \mathcal{I}_{2}, \mathcal{I}_{3}\right)= & p \varphi_{s e}^{+} \otimes|000\rangle\left\langle\left. 000\right|_{z y x}\right. \\
& +(1-p)|0\rangle\left\langle\left. 0\right|_{s} \otimes \tau_{e} \otimes \mid 100\right\rangle\left\langle\left. 100\right|_{z y x},\right.
\end{aligned}
$$

so the reduced system state is biased in the $z$ basis:

$$
\eta_{4}^{s}\left(\mathcal{M}_{1}, \mathcal{I}_{2}, \mathcal{I}_{3}\right)=\frac{p}{2} \mathbb{1}+(1-p)|0\rangle\langle 0| .
$$

We are now in a position to prove the claim that we set out to, namely, that the process considered is genuinely quantum. Demanding classicality means that the experimenter cannot distinguish whether an identity map or a dephasing map was implemented at any subset of previous times. To allow for arbitrary and possibly unrestricted interrogation schemes, here we have considered the more general case where the experimenter is allowed to implement arbitrary CPTP maps, of which any POVM measurement followed by an arbitrary preparation is a special case. This more general notion of classicality (with respect to a general, possibly unrestricted, interrogation scheme) means that the experimenter cannot distinguish between the implementation of the identity map or the CPTP map at any subset of previous times and thereby provides a valid notion of a genuinely quantum process. Above, in Eqs. (I7), (I10), and (I13), we have calculated the system state that would be measured at $t_{4}$ conditioned on the fact that a CPTP map was implemented at each one of the previous three times [as well as the case where only a sequence of identity maps was implemented in Eq. (I5)]. Intuitively, in each of the three cases where an active interrogation is made, the system is biased in one of the $x$-, $y$-, or $z$-basis directions, and in the case where the experimenter interacts only trivially with the system, i.e., implementing a sequence of identity maps, it is completely unbiased. The only way that a measurement at $t_{4}$ cannot distinguish between these four scenarios is if it is blind to biases in every basis. The only types of POVM that can achieve this are trivial, with all elements proportional to the identity matrix, $\left\{\Pi_{4}^{\left(x_{4}\right)}\right\} \propto \mathbb{1} \forall x_{4}$. Thus, there is no (nontrivial) measurement scheme for this process such that the full statistics appears classical, and thus, it is a genuinely quantum process.

[1] A. Streltsov, U. Singh, H. S. Dhar, M. N. Bera, and G. Adesso, Measuring Quantum Coherence with Entanglement, Phys. Rev. Lett. 115, 020403 (2015).

[2] A. Streltsov, G. Adesso, and M. B. Plenio, Colloquium: Quantum Coherence as a Resource, Rev. Mod. Phys. 89, 041003 (2017).

[3] M.-L. Hu, X. Hu, J. Wang, Y. Peng, Y.-R. Zhang, and H. Fan, Quantum Coherence and Geometric Quantum Discord, Phys. Rep. 762-764, 1 (2018).

[4] C. Napoli, T. R. Bromley, M. Cianciaruso, M. Piani, N. Johnston, and G. Adesso, Robustness of Coherence: An
Operational and Observable Measure of Quantum Coherence, Phys. Rev. Lett. 116, 150502 (2016).

[5] I. Marvian and R. W. Spekkens, How to Quantify Coherence: Distinguishing Speakable and Unspeakable Notions, Phys. Rev. A 94, 052324 (2016).

[6] M. Hillery, Coherence as a Resource in Decision Problems: The Deutsch-Jozsa Algorithm and a Variation, Phys. Rev. A 93, 012111 (2016).

[7] J. M. Matera, D. Egloff, N. Killoran, and M. B. Plenio, Coherent Control of Quantum Systems as a Resource Theory, Quantum Sci. Technol. 1, 01LT01 (2016).

[8] S. F. Huelga and M. B. Plenio, Vibrations, Quanta and Biology, Contemp. Phys. 54, 181 (2013).

[9] G. D. Scholes, G. R. Fleming, L. X. Chen, A. AspuruGuzik, A. Buchleitner, D. F. Coker, G. S. Engel, R. van Grondelle, A. Ishizaki, D. M. Jonas et al., Using Coherence to Enhance Function in Chemical and Biophysical Systems, Nature (London) 543, 647 (2017).

[10] L. Wang, M. A. Allodi, and G. S. Engel, Quantum Coherences Reveal Excited-State Dynamics in Biophysical Systems, Nat. Rev. Chem. 3, 477 (2019).

[11] J. Åberg, Quantifying Superposition, arXiv:quant-ph/ 0612146.

[12] T. Baumgratz, M. Cramer, and M. B. Plenio, Quantifying Coherence, Phys. Rev. Lett. 113, 140401 (2014).

[13] F. Levi and F. Mintert, A Quantitative Theory of Coherent Delocalization, New J. Phys. 16, 033007 (2014).

[14] M. Piani, M. Cianciaruso, T. R. Bromley, C. Napoli, N. Johnston, and G. Adesso, Robustness of Asymmetry and Coherence of Quantum States, Phys. Rev. A 93, 042107 (2016).

[15] E. Chitambar and G. Gour, Comparison of Incoherent Operations and Measures of Coherence, Phys. Rev. A 94, 052336 (2016).

[16] A. Winter and D. Yang, Operational Resource Theory of Coherence, Phys. Rev. Lett. 116, 120404 (2016).

[17] E. Chitambar and G. Gour, Critical Examination of Incoherent Operations and a Physically Consistent Resource Theory of Quantum Coherence, Phys. Rev. Lett. 117, 030401 (2016).

[18] E. Chitambar and G. Gour, Quantum Resource Theories, Rev. Mod. Phys. 91, 025001 (2019).

[19] T. Theurer, D. Egloff, L. Zhang, and M. B. Plenio, Quantifying Operations with an Application to Coherence, Phys. Rev. Lett. 122, 190405 (2019).

[20] K.-D. Wu, T. Theurer, G.-Y. Xiang, C.-F. Li, G.-C. Guo, M. B. Plenio, and A. Streltsov, Quantum Coherence and State Conversion: Theory and Experiment, npj Quantum Inf. 6, 22 (2020).

[21] M. M. Wilde, J. M. McCracken, and A. Mizel, Could Light Harvesting Complexes Exhibit Non-classical Effects at Room Temperature?, Proc. R. Soc. A 466, 1347 (2010).

[22] J. S. Briggs and A. Eisfeld, Equivalence of Quantum and Classical Coherence in Electronic Energy Transfer, Phys. Rev. E 83, 051911 (2011).

[23] W. H. Miller, Perspective: Quantum or Classical Coherence?, J. Chem. Phys. 136, 210901 (2012).

[24] R. d. J. León-Montiel and J. P. Torres, Highly Efficient Noise-Assisted Energy Transport in Classical Oscillator Systems, Phys. Rev. Lett. 110, 218101 (2013). 
[25] E. J. O'Reilly and A. Olaya-Castro, Non-classicality of the Molecular Vibrations Assisting Exciton Energy Transfer at Room Temperature, Nat. Commun. 5, 3012 (2014).

[26] For a different demarcation line between classical and quantum physics, based on the memory cost required to simulate a given process, see, e.g., Ref. [27].

[27] C. Budroni, G. Fagundes, and M. Kleinmann, Memory Cost of Temporal Correlations, New J. Phys. 21, 093018 (2019).

[28] A. N. Kolmogorov, Grundbegriffe der Wahrscheinlichkeitsrechnung (Springer, Berlin, 1933); [Foundations of the Theory of Probability (Chelsea, New York, 1956)].

[29] H.-P. Breuer and F. Petruccione, The Theory of Open Quantum Systems (Oxford University Press, Oxford, 2002).

[30] A. J. Leggett and A. Garg, Quantum Mechanics Versus Macroscopic Realism: Is the Flux There When Nobody Looks?, Phys. Rev. Lett. 54, 857 (1985).

[31] A. J. Leggett, Realism and the Physical World, Rep. Prog. Phys. 71, 022001 (2008).

[32] C. Emary, N. Lambert, and F. Nori, Leggett-Garg Inequalities, Rep. Prog. Phys. 77, 016001 (2014).

[33] M. Asano, T. Hashimoto, A. Khrennikov, M. Ohya, and Y. Tanaka, Violation of Contextual Generalization of the Leggett-Garg Inequality for Recognition of Ambiguous Figures, Phys. Scr. T163, 014006 (2014).

[34] A. Smirne, D. Egloff, M. G. Díaz, M. B. Plenio, and S. F. Huelga, Coherence and Non-classicality of Quantum Markov Processes, Quantum Sci. Technol. 4, 01 LT01 (2019).

[35] S. Milz, F. A. Pollock, and K. Modi, An Introduction to Operational Quantum Dynamics, Open Syst. Inf. Dyn. 24, 1740016 (2017).

[36] G. Chiribella, G. M. D'Ariano, and P. Perinotti, Transforming Quantum Operations: Quantum Supermaps, Europhys. Lett. 83, 30004 (2008).

[37] G. Chiribella, G. M. D'Ariano, and P. Perinotti, Theoretical Framework for Quantum Networks, Phys. Rev. A 80, 022339 (2009).

[38] F. A. Pollock, C. Rodríguez-Rosario, T. Frauenheim, M. Paternostro, and K. Modi, Non-Markovian Quantum Processes: Complete Framework and Efficient Characterization, Phys. Rev. A 97, 012127 (2018).

[39] F. A. Pollock, C. Rodríguez-Rosario, T. Frauenheim, M. Paternostro, and K. Modi, Operational Markov Condition for Quantum Processes, Phys. Rev. Lett. 120, 040405 (2018).

[40] P. Taranto, F. A. Pollock, S. Milz, M. Tomamichel, and K. Modi, Quantum Markov Order, Phys. Rev. Lett. 122, 140401 (2019).

[41] P. Taranto, S. Milz, F. A. Pollock, and K. Modi, Structure of Quantum Stochastic Processes with Finite Markov Order, Phys. Rev. A 99, 042108 (2019).

[42] P. Taranto, F. A. Pollock, and K. Modi, Memory Strength and Recoverability of Non-Markovian Quantum Stochastic Processes, arXiv:1907.12583.

[43] P. Taranto, Memory Effects in Quantum Processes, Int. J. Quantum Inf. 18, 1941002 (2020).

[44] P. Strasberg, Operational Approach to Quantum Stochastic Thermodynamics, Phys. Rev. E 100, 022127 (2019).
[45] P. Strasberg and A. Winter, Stochastic Thermodynamics with Arbitrary Interventions, Phys. Rev. E 100, 022135 (2019).

[46] P. Strasberg, Repeated Interactions and Quantum Stochastic Thermodynamics at Strong Coupling, Phys. Rev. Lett. 123, 180604 (2019).

[47] W. Zurek, Einselection and Decoherence from an Information Theory Perspective, Ann. Phys. (Berlin) 9, 855 (2000).

[48] L. Henderson and V. Vedral, Classical, Quantum and Total Correlations, J. Phys. A 34, 6899 (2001).

[49] H. Ollivier and W. H. Zurek, Quantum Discord: A Measure of the Quantumness of Correlations, Phys. Rev. Lett. 88, 017901 (2001).

[50] K. Modi, A. Brodutch, H. Cable, T. Paterek, and V. Vedral, The Classical-Quantum Boundary for Correlations: Discord and Related Measures, Rev. Mod. Phys. 84, 1655 (2012).

[51] G. Chiribella, G. M. D'Ariano, and P. Perinotti, Quantum Circuit Architecture, Phys. Rev. Lett. 101, 060401 (2008).

[52] A. Smirne, T. Nitsche, D. Egloff, S. Barkhofen, S. De, I. Dhand, C. Silberhorn, S. F. Huelga, and M. B. Plenio, Experimental Control of the Degree of Non-classicality via Quantum Coherence, Quantum Sci. Technol. 5, 04LT01 (2020).

[53] G. Chiribella, G. M. D’Ariano, and P. Perinotti, Probabilistic Theories with Purification, Phys. Rev. A 81, 062348 (2010).

[54] W. Feller, An Introduction to Probability Theory and Its Applications (Wiley, New York, 1971).

[55] T. Tao, An Introduction to Measure Theory (American Mathematical Society, Providence, 2011).

[56] S. Milz, F. Sakuldee, F. A. Pollock, and K. Modi, Kolmogorov Extension Theorem for (Quantum) Causal Modelling and General Probabilistic Theories, Quantum 4, 255 (2020).

[57] C. Piron, Ideal Measurement and Probability in Quantum Mechanics, Erkenntnis 16, 397 (1981).

[58] H.-P. Breuer, E.-M. Laine, J. Piilo, and B. Vacchini, Colloquium: Non-Markovian Dynamics in Open Quantum Systems, Rev. Mod. Phys. 88, 021002 (2016).

[59] A. Fine, Hidden Variables, Joint Probability, and the Bell Inequalities, Phys. Rev. Lett. 48, 291 (1982).

[60] T. Heinosaari and M. Ziman, The Mathematical Language of Quantum Theory (Cambridge University Press, Cambridge, England, 2012).

[61] D. Egloff, J. M. Matera, T. Theurer, and M. B. Plenio, $O f$ Local Operations and Physical Wires, Phys. Rev. X 8, 031005 (2018).

[62] M. Lax, Quantum Noise. XI. Multitime Correspondence between Quantum and Classical Stochastic Processes, Phys. Rev. 172, 350 (1968).

[63] H. Carmichael, An Open Systems Approach to Quantum Optics (Springer-Verlag, Berlin, 1993).

[64] C. Gardiner and P. Zoller, Quantum Noise: A Handbook of Markovian and Non-Markovian Quantum Stochastic Methods with Applications to Quantum Optics (Springer, Berlin, 2004). 
[65] V. Gorini, A. Kossakowski, and G. Sudarshan, Completely Positive Semigroups of N-Level Systems, J. Math. Phys. (N.Y.) 17, 821 (1976).

[66] G. Lindblad, On the Generators of Quantum Dynamical Semigroups, Commun. Math. Phys. 48, 119 (1976).

[67] G. Lindblad, Non-Markovian Quantum Stochastic Processes and Their Entropy, Commun. Math. Phys. 65, 281 (1979).

[68] L. Accardi, A. Frigerio, and J. T. Lewis, Quantum Stochastic Processes, Publ. Rest. Inst. Math. Sci. 18, 97 (1982).

[69] L. Li, M. J. Hall, and H. M. Wiseman, Concepts of Quantum Non-Markovianity: A Hierarchy, Phys. Rep. 759, 1 (2018).

[70] Á. Rivas and S. F. Huelga, Open Quantum Systems. An Introduction (Springer, New York, 2012).

[71] M. M. Wolf and J. I. Cirac, Dividing Quantum Channels, Commun. Math. Phys. 279, 147 (2008).

[72] S. Milz, M. Kim, F. A. Pollock, and K. Modi, Completely Positive Divisibility Does Not Mean Markovianity, Phys. Rev. Lett. 123, 040401 (2019).

[73] G. Dümcke, Convergence of Multitime Correlation Functions in the Weak and Singular Coupling Limits, J. Math. Phys. (N.Y.) 24, 311 (1983).

[74] B. Vacchini, A. Smirne, E.-M. Laine, J. Piilo, and H.-P. Breuer, Markovianity and Non-Markovianity in Quantum and Classical Systems, New J. Phys. 13, 093004 (2011).

[75] For a characterization of non-Markovian processes in terms of collections of CPTP maps (or sequences thereof), see Refs. [76,77]. Notably, the characterization employed in these references is equivalent to the one provided here.

[76] F. Sakuldee, S. Milz, F. A. Pollock, and K. Modi, NonMarkovian Quantum Control as Coherent Stochastic Trajectories, J. Phys. A 51, 414014 (2018).

[77] G. A. Paz-Silva, M. J. W. Hall, and H. M. Wiseman, Dynamics of Initially Correlated Open Quantum Systems: Theory and Applications, Phys. Rev. A 100, 042120 (2019).

[78] G. Lindblad, Response of Markovian and Non-Markovian Quantum Stochastic Systems to Time-Dependent Forces (unpublished).

[79] C. Arenz, R. Hillier, M. Fraas, and D. Burgarth, Distinguishing Decoherence from Alternative Quantum Theories by Dynamical Decoupling, Phys. Rev. A 92, 022102 (2015).

[80] A. A. Budini, Conditional Past-Future Correlation Induced by Non-Markovian Dephasing Reservoirs, Phys. Rev. A 99, 052125 (2019).

[81] Á. Rivas, S. F. Huelga, and M. B. Plenio, Quantum NonMarkovianity: Characterization, Quantification and Detection, Rep. Prog. Phys. 77, 094001 (2014).

[82] In contrast to the combs discussed in Refs. [37,51], the combs we consider do not start on an open input line and do not end on an open output line; or, equivalently, in our case, the Hilbert spaces of this initial input and final output space are trivial. Such combs are also called testers in the literature.

[83] D. Kretschmann and R.F. Werner, Quantum Channels with Memory, Phys. Rev. A 72, 062323 (2005).

[84] F. Caruso, V. Giovannetti, C. Lupo, and S. Mancini, Quantum Channels and Memory Effects, Rev. Mod. Phys. 86, 1203 (2014).
[85] F. Costa and S. Shrapnel, Quantum Causal Modelling, New J. Phys. 18, 063032 (2016).

[86] S. Milz, F. A. Pollock, and K. Modi, Reconstructing NonMarkovian Quantum Dynamics with Limited Control, Phys. Rev. A 98, 012108 (2018).

[87] A. Jamiołkowski, Linear Transformations which Preserve Trace and Positive Semidefiniteness of Operators, Rep. Math. Phys. 3, 275 (1972).

[88] M.-D. Choi, Completely Positive Linear Maps on Complex Matrices, Linear Algebra Appl. 10, 285 (1975).

[89] G. Chiribella, G. M. D’Ariano, and P. Perinotti, Memory Effects in Quantum Channel Discrimination, Phys. Rev. Lett. 101, 180501 (2008).

[90] S. Shrapnel, F. Costa, and G. Milburn, Updating the Born Rule, New J. Phys. 20, 053010 (2018).

[91] For $\chi=0, C_{K}$ is actually not a proper comb, as it does not satisfy the hierarchy of trace conditions that ensure causal ordering. Nonetheless, this lack of causality could not be picked up by means of projective measurements in the classical basis alone and thus does not pose a problem for our discussion.

[92] A. Ferraro, L. Aolita, D. Cavalcanti, F. M. Cucchietti, and A. Acín, Almost All Quantum States Have Nonclassical Correlations, Phys. Rev. A 81, 052318 (2010).

[93] S. Boyd and L. Vandenberghe, Convex Optimization (Cambridge University Press, Cambridge, England, 2004).

[94] M. Horodecki, P. Horodecki, and R. Horodecki, Separability of Mixed States: Necessary and Sufficient Conditions, Phys. Lett. A 223, 1 (1996).

[95] K. M. R. Audenaert and M. B. Plenio, When Are Correlations Quantum?-Verification and Quantification of Entanglement by Simple Measurements, New J. Phys. 8, 266 (2006).

[96] J. Eisert, F. G. S. L. Brandão, and K. M. R. Audenaert, Quantitative Entanglement Witnesses, New J. Phys. 9, 46 (2007).

[97] O. Gühne, M. Reimpell, and R. F. Werner, Estimating Entanglement Measures in Experiments, Phys. Rev. Lett. 98, 110502 (2007).

[98] C. M. Li, N. Lambert, Y. N. Chen, G. Y. Chen, and F. Nori, Witnessing Quantum Coherence: From Solid-State to Biological Systems, Sci. Rep. 2, 885 (2012).

[99] A. Datta, A Condition for the Nullity of Quantum Discord, arXiv:1003.5256.

[100] B. Yadin, J. Ma, D. Girolami, M. Gu, and V. Vedral, Quantum Processes Which Do Not Use Coherence, Phys. Rev. X 6, 041028 (2016).

[101] J. Åberg, Catalytic Coherence, Phys. Rev. Lett. 113, 150402 (2014).

[102] Note that the term "device independent" does not imply that one needs no assumptions on the devices but just that the assumptions are trivial. In the case of device-independent quantum key distributions, for instance, the assumption that the devices have some kind of independence needs to be assumed. In our case, we need to assume that the measurements are not trivial because otherwise there is nothing one can find from their statistics.

[103] A. Acín, N. Brunner, N. Gisin, S. Massar, S. Pironio, and V. Scarani, Device-Independent Security of Quantum 
Cryptography against Collective Attacks, Phys. Rev. Lett. 98, 230501 (2007).

[104] V. Scarani, The Device-Independent Outlook on Quantum Physics, Acta Phys. Slovaca 62, 347 (2012).

[105] H. Liu, W. Wang, K. Wei, X.-T. Fang, L. Li, N.-L. Liu, H. Liang, S.-J. Zhang, W. Zhang, H. Li et al., Experimental Demonstration of High-Rate Measurement-Device-Independent Quantum Key Distribution over Asymmetric Channels, Phys. Rev. Lett. 122, 160501 (2019).

[106] J. Kołodyński, A. Máttar, P. Skrzypczyk, E. Woodhead, D. Cavalcanti, K. Banaszek, and A. Acín, Device-Independent Quantum Key Distribution with Single-Photon Sources, Quantum 4, 260 (2020).

[107] For experiments that detect somewhat macroscopic superpositions, see, e.g., Refs. [108-110].

[108] O. Nairz, M. Arndt, and A. Zeilinger, Quantum Interference Experiments with Large Molecules, Am. J. Phys. 71, 319 (2003).

[109] A. D. O’Connell, M. Hofheinz, M. Ansmann, R. C. Bialczak, M. Lenander, E. Lucero, M. Neeley, D. Sank, H. Wang, M. Weides et al., Quantum Ground State and Single-Phonon Control of a Mechanical Resonator, Nature (London) 464, 697 (2010).

[110] S. Eibenberger, S. Gerlich, M. Arndt, M. Mayor, and J. Tüxen, Matter-Wave Interference of Particles Selected from a Molecular Library with Masses Exceeding 10 000 amu, Phys. Chem. Chem. Phys. 15, 14696 (2013).

[111] W. H. Zurek, Decoherence, Einselection, and the Quantum Origins of the Classical, Rev. Mod. Phys. 75, 715 (2003).

[112] M. Schlosshauer, Decoherence, the Measurement Problem, and Interpretations of Quantum Mechanics, Rev. Mod. Phys. 76, 1267 (2005).

[113] S. Nakajima, On Quantum Theory of Transport Phenomena: Steady Diffusion, Progr. Theor. Phys. 20, 948 (1958).

[114] R. Zwanzig, Ensemble Method in the Theory of Irreversibility, J. Chem. Phys. 33, 1338 (1960).

[115] W.-M. Zhang, P.-Y. Lo, H.-N. Xiong, M. W.-Y. Tu, and F. Nori, General Non-Markovian Dynamics of Open Quantum Systems, Phys. Rev. Lett. 109, 170402 (2012).

[116] A. Chakraborty and R. Sensarma, Power-Law Tails and Non-Markovian Dynamics in Open Quantum Systems: An Exact Solution from Keldysh Field Theory, Phys. Rev. B 97, 104306 (2018).
[117] R. Demkowicz-Dobrzański, M. Jarzyna, and J. Kołodyński, Quantum Limits in Optical Interferometry, Prog. Opt. 60, 345 (2015).

[118] A. Smirne, J. Kołodyński, S. F. Huelga, and R. DemkowiczDobrzański, Ultimate Precision Limits for Noisy Frequency Estimation, Phys. Rev. Lett. 116, 120801 (2016).

[119] J. F. Haase, A. Smirne, S. F. Huelga, J. Kołodynski, and R. Demkowicz-Dobrzanski, Precision Limits in Quantum Metrology with Open Quantum Systems, Quantum Meas. Quantum Metrol. 5, 13 (2016).

[120] Y. Yang, Memory Effects in Quantum Metrology, Phys. Rev. Lett. 123, 110501 (2019).

[121] J. Gemmer, A. Otte, and G. Mahler, Quantum Approach to a Derivation of the Second Law of Thermodynamics, Phys. Rev. Lett. 86, 1927 (2001).

[122] J. Prior, A. W. Chin, S. F. Huelga, and M. B. Plenio, Efficient Simulation of Strong System-Environment Interactions, Phys. Rev. Lett. 105, 050404 (2010).

[123] D. Tamascelli, R. Rosenbach, and M. B. Plenio, Improved Scaling of Time-Evolving Block-Decimation Algorithm through Reduced-Rank Randomized Singular Value Decomposition, Phys. Rev. E 91, 063306 (2015).

[124] T. Farrelly, F. G. S. L. Brandão, and M. Cramer, Thermalization and Return to Equilibrium on Finite Quantum Lattice Systems, Phys. Rev. Lett. 118, 140601 (2017).

[125] D. Tamascelli, A. Smirne, J. Lim, S. F. Huelga, and M. B. Plenio, Efficient Simulation of Finite-Temperature Open Quantum Systems, Phys. Rev. Lett. 123, 090402 (2019).

[126] D. de Falco and D. Tamascelli, Noise-Assisted Quantum Transport and Computation, J. Phys. A 46, 225301 (2013).

[127] P. Strasberg and M. G. Díaz, Classical Quantum Stochastic Processes, Phys. Rev. A 100, 022120 (2019).

[128] Namely, that modeling the statistics as coming from an evolution satisfying NCGD is equivalent to modeling it as coming from a classical evolution.

[129] B. Baumgartner and H. Narnhofer, Analysis of Quantum Semigroups with GKS-Lindblad Generators: II. General, J. Phys. A 41, 395303 (2008).

[130] W. F. Stinespring, Positive Functions on $C^{*}$-Algebras, Proc. Am. Math. Soc. 6, 211 (1955). 